Supporting Information for

\title{
Prioritizing Direct Photolysis Products Predicted by the Chemical Transformation Simulator: Relative Reasoning and Absolute Ranking
}

Chenyi Yuan ${ }^{\mathrm{a}}$, Caroline Tebes-Stevens ${ }^{\mathrm{b}^{*}}$, Eric J. Weber ${ }^{\mathrm{b}}$

${ }^{a}$ Oak Ridge Institute for Science and Education (ORISE), hosted at United States Environmental Protection Agency, Athens, Georgia 30605, United States

${ }^{\mathrm{b}}$ Center for Environmental Measurement and Modeling, United States Environmental Protection Agency, Athens, Georgia 30605, United States

Number of Pages: 49

Number of Texts: 3

Number of Tables: 8

Number of Figures: 3 
Table of Content

\begin{tabular}{|c|c|c|}
\hline Text S1 & Selection and uncertainty of the standardization factor $\beta$. & S3-S4 \\
\hline Text S2 & Calculation and variation of the formation ratio $\alpha$. & S5-S6 \\
\hline Text S3 & Metabolizer algorithm. & S7-S10 \\
\hline Table S1 & Parent degradation and product formation rates from DB-J-ENV. & S11-S20 \\
\hline Table S2 & Parent degradation and product formation rates from DB-EFSA-ENV. & S21-S29 \\
\hline Table S3 & $\begin{array}{l}\text { Rank levels of all reaction schemes in the direct photolysis reaction } \\
\text { library v1.3 and the supporting statistics. }\end{array}$ & S30-S36 \\
\hline Table S4 & $\begin{array}{l}\text { Internal evaluation of each version of the direct photolysis library } \\
\text { against DB-J-ENV revealing step-wise improvement. }\end{array}$ & S37 \\
\hline Table S5 & $\begin{array}{l}\text { External evaluation of each version of the direct photolysis library } \\
\text { against DB-EFSA-ENV revealing step-wise improvement. }\end{array}$ & S38 \\
\hline Table S6 & $\begin{array}{l}\text { Improvement of evaluation measures after modification of } \\
\text { "trifluoromethyl photohydrolysis" for data in DB-J-ENV. }\end{array}$ & S39 \\
\hline Table S7 & $\begin{array}{l}\text { Improvement of evaluation measures after modification of } \\
\text { "trifluoromethyl photohydrolysis" for data in DB-EFSA-ENV. }\end{array}$ & $\mathrm{S} 40$ \\
\hline Table S8 & List of acronyms. & S41 \\
\hline Figure S1 & $\begin{array}{l}\text { Differences of compiled parent degradation half-lives for the same } \\
\text { compound from different studies. }\end{array}$ & S42 \\
\hline Figure S2 & $\begin{array}{l}\text { Change in precision for reaction schemes after applying relative } \\
\text { reasoning rules (a, from library v1.0 to v1.2) and adding ranking } \\
\text { levels (b, from library v1.2 to v1.3) for data from DB-EFSA-ENV. }\end{array}$ & $\mathrm{S} 43$ \\
\hline Figure S3 & $\begin{array}{l}\text { Comparison of the geometric mean of half-lives of the same reaction } \\
\text { scheme }\left(\mathrm{t}_{1 / 2, \mathrm{pr}}\right) \text { in the two databases. }\end{array}$ & S44 \\
\hline References & & $\mathrm{S} 45-\mathrm{S} 49$ \\
\hline
\end{tabular}


Text S1. Selection and uncertainty of the standardization factor $\beta$.

The standardization factor $\beta$ was created to standardize studies of the two following experimental conditions to a light intensity of daily mid-summer (June $21^{\text {st }}$, summer solstice) light at $40{ }^{\circ} \mathrm{N}$ latitude as best as feasible. $k_{\mathrm{pa}}=k_{\mathrm{pa} \text {,reported }} / \beta$, and $t_{1 / 2, \mathrm{pa}}=t_{1 / 2 \text {,pa,reported } \beta} \beta$ is multipliable when multiple adjustments are needed.

The first condition is that the solution of the parent contaminant was photolyzed under natural sunlight. The $\beta$ value needs to standardize both the daily and seasonal light variation. The below table shows the ratio of midday (solar noon) sunlight intensity over daily (24-hour-averaged) sunlight intensity (in mole of photons) at $40{ }^{\circ} \mathrm{N}$ latitude at different dates of the year and for different wavelength ranges according to data collected by Apell et al. ${ }^{1}$ We selected a unified factor of 3.3 to approximate the difference between midday and daily sunlight, because the number is within the ratio range during spring to fall and at UVA wavelengths, which is most common for the referenced studies. For studies which only report results irradiated during the day without specifying the time of the day, we divided the factor of 3.3 by 2 to approximate the weakened light in the morning or afternoon (i.e. a $\beta$ value of 1.7).

\begin{tabular}{lllll}
\hline wavelength range & $\begin{array}{l}\text { Jun } 21^{\text {st }} \\
\text { noon/daily }\end{array}$ & $\begin{array}{l}\text { Mar 20 } \\
\text { noon/daily }\end{array}$ & $\begin{array}{l}\text { Sep 22 } \\
\text { noon/daily }\end{array}$ & $\begin{array}{l}\text { Dec 22 } \\
\text { noon/daily }\end{array}$ \\
\hline UVB $(280-315 \mathrm{~nm})$ & 3.7 & 4.4 & 4.3 & 5.7 \\
UVA $(315-400 \mathrm{~nm})$ & 2.9 & 3.5 & 3.5 & 4.4 \\
visible $(400-800 \mathrm{~nm})$ & 2.7 & 3.3 & 3.3 & 4.3 \\
\hline
\end{tabular}

Similarly, the ratio of the daily sunlight intensity at a certain date over that on June $21^{\text {st }}$ at $40{ }^{\circ} \mathrm{N}$ latitude is shown in the following table. Because that the ratios for spring, summer, and fall are similar, we only applied a $\beta$ value of 0.3 for the winter season.

\begin{tabular}{lllll}
\hline Wavelength range & $\begin{array}{l}\text { Jun 21 } \\
\text { daily }\end{array}$ & $\begin{array}{l}\text { Mar 20th } 21^{\text {st }} \\
\text { daily 21 }\end{array}$ & $\begin{array}{l}\text { Sep 22 } \\
\text { daily }\end{array}$ & $\begin{array}{l}\text { Dec 22 } \\
\text { daily }\end{array}$ \\
\hline UVB $(280-315 \mathrm{~nm})$ & 1.0 & 0.5 & 0.6 & 0.1 \\
UVA $(315-400 \mathrm{~nm})$ & 1.0 & 0.6 & 0.6 & 0.3 \\
visible $(400-800 \mathrm{~nm})$ & 1.0 & 0.7 & 0.7 & 0.3 \\
\hline
\end{tabular}

Uncertainty in $\beta$ estimation for this experimental condition depends on the exact time-span of irradiation and wavelengths to be absorbed by the contaminant, which are mostly unknown. Other uncertainty can come from latitude, temperature, and cloud cover.

The second condition is that the solution of the parent contaminant was photolyzed in a solar simulator equipped with a filtered xenon lamp. Ideally, such a filtered solar simulator greatly resembles solar light. With a usual setting of $500 \mathrm{~W} \mathrm{~m}^{-2}$ in an Atlas Suntest solar simulator, the measured light intensity is similar to noon sunlight on June $21^{\text {st }}$, especially in the range of UVA region. ${ }^{2}$ The measured light intensity is $474 \mathrm{~W} \mathrm{~m}^{-2}$ for $280-800 \mathrm{~nm}$ and $59 \mathrm{~W} \mathrm{~m}^{-2}$ for $280-400$ $\mathrm{nm},{ }^{2}$ and therefore the light intensity resembling daily sunlight on Jun $21^{\text {st }}$ should be around 150 
$\mathrm{W} \mathrm{m} \mathrm{m}^{-2}$ for $280-800 \mathrm{~nm}$ and $18 \mathrm{~W} \mathrm{~m}^{-2}$ for $280-400 \mathrm{~nm}$ using the above standardization factor of 3.3. Therefore, when a referenced study in the database reports the light intensity of filtered xenon lamp, the light intensity is standardized to $150 \mathrm{~W} \mathrm{~m}^{-2}$ or $18 \mathrm{~W} \mathrm{~m}^{-2}$ depending on the given wavelength range and the $\beta$ value is calculated as the ratio accordingly. Uncertainty for this experimental condition mostly comes from the errors in the exact wavelengths to be absorbed by the contaminant and in measuring the light intensity in the solar simulator. 
Text S2. Calculation and variation of the formation ratio $\alpha$.

\section{Calculation of $\alpha$}

Two assumptions were used to estimate $\alpha$.

Assumption 1: products do not degrade further.

Assumption 2: the photolysis reaction falls in the initial reaction period.

Although the assumptions may not always be valid, but limited data prohibited a more accurate estimation.

The kinetics equations of a parent compound $(\mathrm{Pa})$ photodegrading to a couple of product compounds $\left(\operatorname{Pr}_{i}, i=1,2,3 \ldots\right)$ are written as follows.

$$
\begin{aligned}
& P a \stackrel{k_{p r, i}}{\longrightarrow} P r_{i} \\
& k_{p a}=\sum k_{p r, i} \\
& \frac{d[P a]}{d t}=-k_{p a}[P a] \\
& \frac{d\left[P r_{i}\right]}{d t}=k_{p r, i}[P a] \\
& {[P a]_{t}=[P a]_{0} e^{-k_{p a} t} \approx[P a]_{0}\left(1-k_{p a} t\right)} \\
& {\left[P r_{i}\right]_{t}=\left[P r_{i}\right]_{0}+\frac{k_{p r, i}}{k_{p a}}[P a]_{0}\left(1-e^{-k_{p a} t}\right) \approx\left[P r_{i}\right]_{0}+\frac{k_{p r, i}}{k_{p a}}[P a]_{0} k_{p a} t} \\
& \alpha_{i}=\frac{k_{p r, i}}{k_{p a}} \approx \frac{\left[P r_{i}\right]_{t}-\left[P r_{i}\right]_{0}}{[P a]_{0}-[P a]_{t}}
\end{aligned}
$$

Therefore, the ratio of the concentration change of the product over that of the parent was used as an estimate of the formation ratio. We estimated $\alpha$ using a single point from the degradation kinetics data (from text, table, or figure) for compounds in DB-J-ENV, with a preference for a time-point where the formation of the major product was close to its maximum. This approach assured the readability of the data to be extracted and avoided using data in the product decay region. Since EFSA reports usually tabulate kinetics data that are easy to extract, we estimated $\alpha$ using the mean value from all valid data points $(0 \leq$ ratio $\leq 1)$ from the degradation curve for compounds in DB-EFSA-ENV.

\section{Variation of $\alpha$}

In order to understand the difference between chosing one single time-point and using the average ratio calculated for all time-points, the compiled kinetics data from DB-EFSA-ENV were explored for a simple estimation. The following figure plotted the maximum ratio devided by the average of all ratios calculated for all time-points (max/avg), and the minumum ratio devidede by the same average ( $\mathrm{min} / \mathrm{avg}$ ), for each informative combination of a parent 
compound and a reaction scheme. As seen from the figure, the difference caused by using a single time-point versus all time-points are usally within 10-fold.

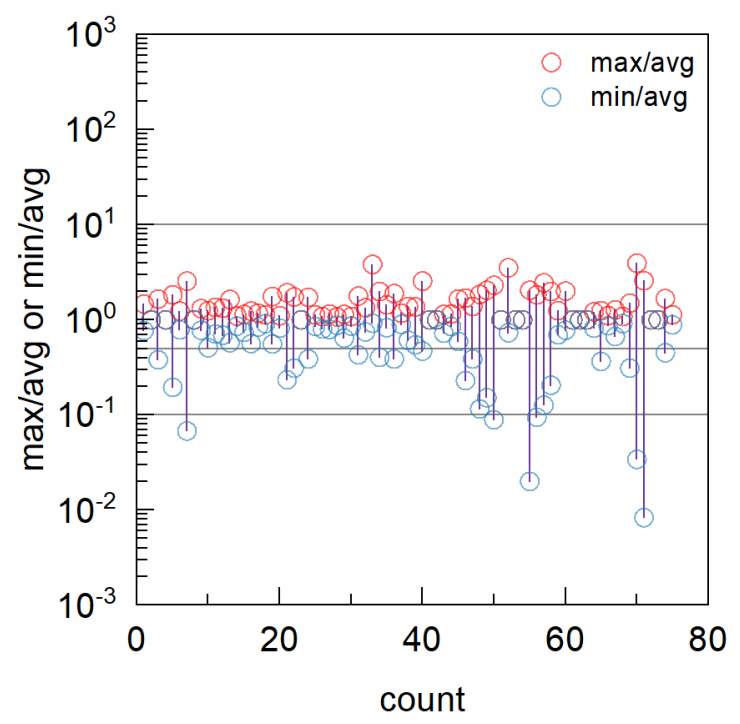


Text S3. Metabolizer algorithm.

This is updated from the "description of ChemAxon Metabolizer algorithm" section in the supporting information of our earlier paper on hydrolysis reaction library. ${ }^{3}$

The Chemical Transformation Simulator (CTS) module for prediction of transformation products is built on ChemAxon's Metabolizer application. ${ }^{4}$ In Metabolizer, reaction products are predicted using a library of reaction schemes. Each reaction scheme is assigned a fixed rank within the library. Ranks are a dimensionless measure of the rate of the reaction scheme relative to other schemes in the library. The ranks are used to calculate a "formation" of the product(s) formed according to the scheme. This formation is then used to calculate a "production", an "accumulation", a "global production", and a "global accumulation" of each product in percentage.

\section{The algorithm to calculate "production", "accumulation", "global production", and "global accumulation"}

The algorithm implemented in Metabolizer is based on first-order transformation kinetics and is best illustrated with a simple example. Consider a parent chemical $\mathrm{C}$ which is transformed into three possible degradation products, $\mathrm{D}_{1}, \mathrm{D}_{2}$ and $\mathrm{D}_{3}$, through reactions with rate constants of $\mathrm{k}_{1}$, $k_{2}$ and $k_{3}$, respectively, and $D_{1}$ and $D_{2}$ are further transformed to $D_{11}, D_{12}, D_{21}$ and then to $D_{111}$ :

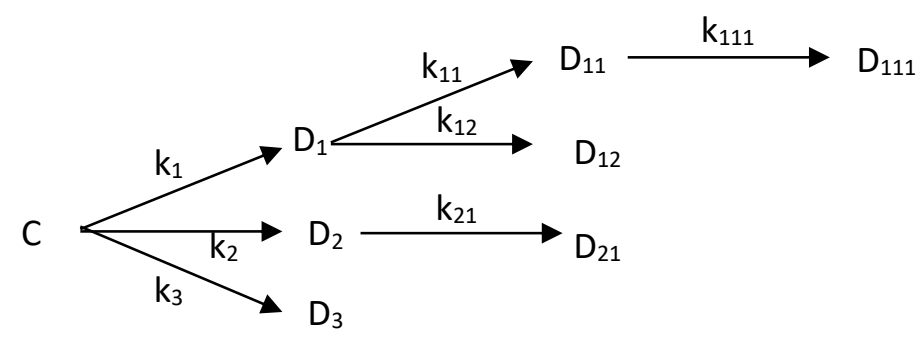

The overall rate of change in the concentration of the parent chemical is given by:

$$
\frac{d[C]}{d t}=-\left(k_{1}+k_{2}+k_{3}\right)[C]
$$

The rate of change in the concentration of each product $D_{i}$ in the first generation is given by:

$$
\frac{d\left[D_{i}\right]}{d t}=k_{i}[C]
$$

Equations (1) and (2) can be integrated to give the concentration of the parent and the transformation products as a function of time:

$$
\begin{aligned}
& {[C]=[C]_{0} \mathrm{e}^{-\left(k_{1}+k_{2}+k_{3}\right) \mathrm{t}}} \\
& {\left[D_{i}\right]=\frac{k_{i}[C]_{0}}{k_{1}+k_{2}+k_{3}}\left(1-\mathrm{e}^{-\left(k_{1}+k_{2}+k_{3}\right) \mathrm{t}}\right)}
\end{aligned}
$$


where $[\mathrm{C}]_{0}$ is the concentration of the parent at time zero. Equations (3) and (4) can be combined to show that at any given time, the percent portion of $\mathrm{C}$ degradation that is due to $\mathrm{D}_{\mathrm{i}}$ production is given by:

$$
P_{i}=\frac{100\left[D_{i}\right]}{[C]_{0}-[C]} \%=\frac{100 k_{i}}{k_{1}+k_{2}+k_{3}} \%
$$

where $P_{i}$ is termed the "production" of $D_{i}$. This equation can be generalized for transformation of a chemical that is transformed according to $\mathrm{N}$ reactions as follows:

$$
P_{i}=\frac{100 k_{i}}{\sum_{j=1}^{N} k_{j}} \%
$$

The idea of the product accumulation calculation is based on comparing the rate constant of the process that forms $D_{i}\left(i . e . k_{i}\right)$ and the rate constants of the $N_{i}$ processes that degrade $D_{i}\left(k_{i 1}, k_{i 2}\right.$, $\ldots, \mathrm{k}_{\mathrm{i} l}$, where $l=\mathrm{N}_{\mathrm{i}}$ ). Specifically, the "accumulation" of $\mathrm{D}_{\mathrm{i}}$ is defined as follows.

$$
A_{i}=\max \left(\frac{k_{i}-\sum_{l=1}^{N_{i}} k_{i l}}{k_{i}} P_{i}, 0\right)
$$

where $\max ()$ returns the maximum value of the two values inside the parenthesis. In the above example, product $\mathrm{D}_{1}$ is formed from a reaction with a rate constant of $\mathrm{k}_{1}$ and is degraded through reactions with rate constants of $\mathrm{k}_{11}$ and $\mathrm{k}_{12}$, and according to Equation (7), the "accumulation" of $\mathrm{D}_{1}$, or $\mathrm{A}_{1}$ (the symbol used in equation (7)), is calculated as follows:

$$
A_{1}=\max \left(\frac{k_{1}-\left(k_{11}+k_{12}\right)}{k_{1}} P_{1}, 0\right)
$$

Similarly, $\mathrm{A}_{2}$ and $\mathrm{A}_{3}$ are calculated according to the following equations:

$$
\begin{aligned}
& A_{2}=\max \left(\frac{k_{2}-k_{21}}{k_{2}} P_{2}, 0\right) \\
& A_{3}=\max \left(\frac{k_{3}}{k_{3}} P_{3}, 0\right)
\end{aligned}
$$

It is noteworthy that when an "accumulation" is calculated to be 0 , it will be displayed as $<0.001 \%$ in CTS.

The "production" and "accumulation" of the next generation product $\mathrm{D}_{\mathrm{im}}$ which is formed from the reaction with a rate constant of $\mathrm{k}_{\mathrm{im}}$ and is degraded through $\mathrm{N}_{\mathrm{im}}$ reactions with rate constants of $\mathrm{k}_{\mathrm{im} 1}, \mathrm{k}_{\mathrm{im} 2}, \ldots, \mathrm{k}_{\mathrm{imq}}$ (where $\mathrm{q}=\mathrm{N}_{\mathrm{im}}$ ) can be calculated in an iterative manner from the "production" and "accumulation" of the product of the previous generations:

$$
\begin{aligned}
P_{i m} & =\frac{\left(P_{i}-A_{i}\right) k_{i m}}{\sum_{l=1}^{N_{i}} k_{i l}} \\
A_{i m} & =\max \left(\frac{k_{i m}-\sum_{q=1}^{N_{i m}} k_{i m q}}{k_{i m}} P_{i m}, 0\right)
\end{aligned}
$$


For example, the "production" and "accumulation" of $\mathrm{D}_{11}$ which is formed from a reaction with a rate constant of $\mathrm{k}_{11}$ and is degraded through a reaction with a rate constant of $\mathrm{k}_{111}$ can be calculated as follows:

$$
\begin{aligned}
& P_{11}=\frac{\left(P_{1}-A_{1}\right) k_{11}}{k_{1}+k_{2}} \\
& A_{11}=\max \left(\frac{k_{11}-k_{111}}{k_{11}} P_{11}, 0\right)
\end{aligned}
$$

Equation 6 can also be generalized in this manner recognizing that the production of $\mathrm{C}$ is $100 \%$ (P) and accumulation is $0 \%(\mathrm{~A})$ :

$$
P_{i}=\frac{100 k_{i}}{\sum_{j=1}^{N} k_{j}} \%=\frac{(P-A) k_{i}}{\sum_{j=1}^{N} k_{j}}
$$

When a same product is formed by different processes in the degradation tree, the "global production" is calculated as the sum of all the relevant "production" and the "global accumulation" is calculated as the sum of all the relevant "accumulation". For example, if $\mathrm{D}_{12}$ and $\mathrm{D}_{21}$ have the same structure in the above example, then the "global production" of $\mathrm{D}_{12}$ or $\mathrm{D}_{21}$, termed as $\mathrm{GP}_{12}$ or $\mathrm{GP}_{21}$, is equal to $\mathrm{P}_{12}+\mathrm{P}_{21}$; and "global accumulation" of $\mathrm{D}_{12}$ or $\mathrm{D}_{21}$, termed as $\mathrm{GA}_{12}$ or $\mathrm{GA}_{21}$, is equal to $\mathrm{A}_{12}+\mathrm{A}_{21}$.

\section{The system of "rank" calculated based on "formation" as a proxy of rate constants.}

If rate constants were available for each transformation reaction, it would be possible to calculate an exact percent production for each product using the above equations. In general, however, neither measured rate constants, nor algorithms to estimate rate constants are available for many transformation reactions.

In Metabolizer, an algorithm has been implemented to approximate a percent production for products of each reaction scheme by categorizing the rate constant of each scheme with an integer rank on a scale of one to seven. A rank of seven is assigned to the fastest reaction schemes and a rank of one is assigned to the slowest reaction schemes. In the Metabolizer algorithm, the unitless "formation" value for scheme $i\left(f_{i}\right)$ is defined as the number 7 raised to the power of its rank $\left(\operatorname{rank}_{\mathrm{i}}\right)$ :

$$
f_{i} \stackrel{\text { def }}{=} 7^{r a n k_{i}}
$$

The "formation" values are analogous to rate constants, and the "production" and "accumulation" in the Equations (1) to (15) can be calculated by substituting all rate constants with "formation". For example, Equation (6) which describes the "production" of $\mathrm{D}_{\mathrm{i}}$ generated by scheme $i$ is calculated according to the following equation:

$$
P_{i}=\frac{100 f_{i}}{\sum_{j=1}^{N} f_{j}} \%
$$

The "formation" values used in Metabolizer have no units; however, for the libraries that we developed in-house, we associate our rank assignments with particular half-life ranges. To be consistent with the Metabolizer algorithm, the half-life ranges were selected so that the rate 
constants associated with the midpoints of each half-life range would be roughly proportional to the "formation" and thus also lie along a line that was an exponential function of the rank:

$$
f_{i} \propto k_{i}=a \times 7^{\text {rank }_{i}}
$$

Where $\mathrm{a}$ is a selected constant and is around 0.0001 in our case.

The figure below shows how formation values (plotted on the left vertical axis) and rateconstants (plotted on the right vertical axis) vary as a function of rank.

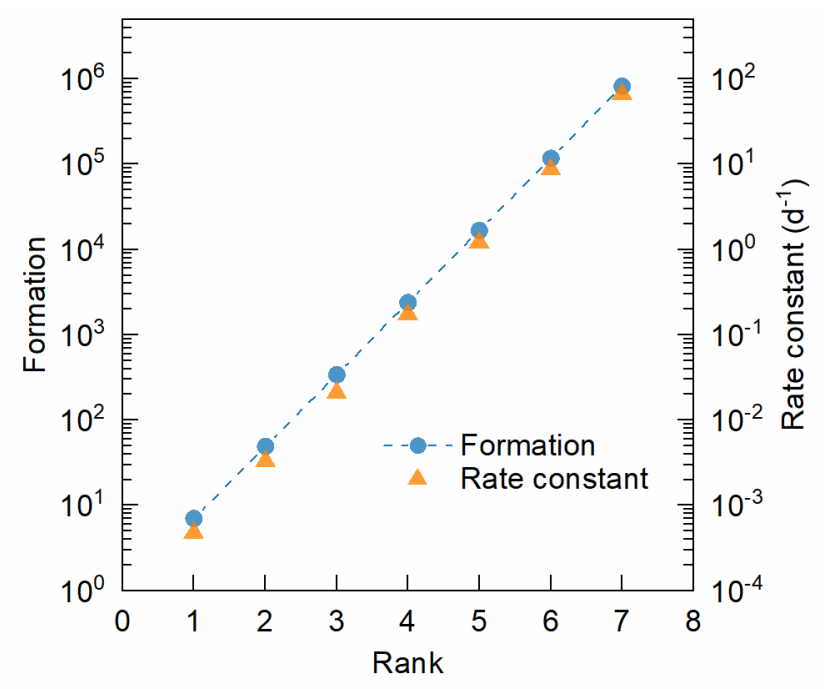

The following table presents the numerical values used to generate the plot:

\begin{tabular}{ccccc}
\hline rank & formation & half-life range $(\mathrm{d})$ & midpoint half-life ${ }^{\mathrm{a}}(\mathrm{d})$ & midpoint rate constant $\left(\mathrm{d}^{-1}\right)$ \\
\hline 1 & 7 & $>365$ & $1.5 \times 10^{3}$ & 0.00048 \\
2 & 49 & $60-365$ & $2.1 \times 10^{2}$ & 0.0033 \\
3 & 343 & $7-60$ & 34 & 0.021 \\
4 & 2401 & $1-7$ & 4.0 & 0.17 \\
5 & 16807 & $0.14-1$ & 0.57 & 1.2 \\
6 & 117649 & $0.02-0.14$ & 0.080 & 8.7 \\
7 & 823543 & $<0.02$ & 0.010 & 67 \\
\hline
\end{tabular}

Note: ${ }^{a}$ midpoint half-life is calculated as the mean of the borders of the half-life range for ranks $2-6$, half of the upper border $(0.02 \mathrm{~d})$ for rank 1 , and the mean of the lower border $(365 \mathrm{~d})$ and the lower border multiplied by $7(365 \mathrm{~d} \times 7)$ for rank 7 .

This above table can be used to verify that the percent production values calculated with the rate constants are roughly the same as the percent production values calculated with the formation values. For example, for a chemical that can be transformed according to three reaction schemes with ranks 6, 4 and 2, the production percentages are $98-99 \%, 1-2 \%$ and $<0.1 \%$, respectively, whether these percentages are calculated with the formation values or the midpoint rate constants. 
Table S1. Parent degradation and product formation rates from DB-J-ENV.

\begin{tabular}{|c|c|c|c|c|c|c|c|c|c|}
\hline reference & contaminant & reaction scheme & $\beta$ & $\begin{array}{l}k_{\mathrm{pa}} \\
\left(\mathrm{s}^{-1}\right)\end{array}$ & $\begin{array}{l}t_{1 / 2, \mathrm{pa}} \\
(\mathrm{s})\end{array}$ & $\begin{array}{l}t_{1 / 2, \text { pa,avg }} \\
(\mathrm{s})\end{array}$ & $\alpha$ & $\begin{array}{l}k_{\text {pr,avg }} \\
\left(\mathrm{s}^{-1}\right)\end{array}$ & $\begin{array}{l}t_{1 / 2, \text { pr,avg }} \\
(\mathrm{s})\end{array}$ \\
\hline Nilles $1974^{5}$ & basalin & $\begin{array}{l}\text { dinitroaniline photochemical N- } \\
\text { dealkylation }\end{array}$ & 1.00 & $1.6 \mathrm{E}-6$ & $4.2 \mathrm{E}+5$ & $4.2 \mathrm{E}+5$ & 0.10 & $1.6 \mathrm{E}-7$ & $4.5 \mathrm{E}+6$ \\
\hline Wammer $2013^{6}$ & enrofloxacin & $\begin{array}{l}\text { fluoroquinolone photochemical } \\
\text { N-dealkylation }\end{array}$ & 3.33 & $1.7 \mathrm{E}-3$ & $4.0 \mathrm{E}+2$ & $3.8 \mathrm{E}+2$ & 0.05 & $2.8 \mathrm{E}-4$ & $2.5 \mathrm{E}+3$ \\
\hline Fasani $1999^{7}$ & norfloxacin & $\begin{array}{l}\text { fluoroquinolone fluoride } \\
\text { photohydrolysis }\end{array}$ & & & & $4.1 \mathrm{E}+2$ & 1.00 & $1.7 \mathrm{E}-3$ & $4.1 \mathrm{E}+2$ \\
\hline Kusari $2009^{8}$ & difloxacin & $\begin{array}{l}\text { fluoroquinolone photochemical } \\
\text { N-dealkylation }\end{array}$ & & & & $4.4 \mathrm{E}+2$ & 0.33 & $5.2 \mathrm{E}-4$ & $1.3 \mathrm{E}+3$ \\
\hline $\begin{array}{l}\text { Burhenne } \\
1997^{9}\end{array}$ & enrofloxacin & $\begin{array}{l}\text { fluoroquinolone photochemical } \\
\text { N-dealkylation }\end{array}$ & & & & $3.8 \mathrm{E}+2$ & 0.26 & $2.8 \mathrm{E}-4$ & $2.5 \mathrm{E}+3$ \\
\hline Burhenne 1997 & enrofloxacin & $\begin{array}{l}\text { fluoroquinolone piperazine } \\
\text { photochemical bis N-dealkylation }\end{array}$ & & & & $3.8 \mathrm{E}+2$ & 0.32 & 5.7E-4 & $1.2 \mathrm{E}+3$ \\
\hline Boreen $2005^{10}$ & sulfamethazine & $\begin{array}{l}\text { sulfonamide SO2 extrusion } \\
\text { photorearrangement (6-6) }\end{array}$ & 3.33 & $1.9 \mathrm{E}-6$ & $3.7 \mathrm{E}+5$ & $1.5 \mathrm{E}+5$ & 0.64 & $2.9 \mathrm{E}-6$ & $2.4 \mathrm{E}+5$ \\
\hline Boreen $2004^{11}$ & sulfamethoxazole & sulfonamide photohydrolysis & 3.33 & $3.9 \mathrm{E}-6$ & $1.8 \mathrm{E}+5$ & $6.6 \mathrm{E}+4$ & 0.35 & $2.0 \mathrm{E}-6$ & $3.5 \mathrm{E}+5$ \\
\hline Boreen 2004 & \begin{tabular}{|l|} 
sulfisoxazole \\
\end{tabular} & sulfonamide photohydrolysis & 3.33 & $7.5 \mathrm{E}-6$ & $9.2 \mathrm{E}+4$ & $6.0 \mathrm{E}+4$ & 0.66 & $7.6 \mathrm{E}-6$ & $9.2 \mathrm{E}+4$ \\
\hline Boreen 2004 & sulfamethizole & sulfonamide photohydrolysis & 3.33 & $3.9 \mathrm{E}-6$ & $1.8 \mathrm{E}+5$ & $1.1 \mathrm{E}+5$ & 0.70 & $4.3 \mathrm{E}-6$ & $1.6 \mathrm{E}+5$ \\
\hline Boreen 2004 & sulfathiazole & sulfonamide photohydrolysis & 3.33 & $1.7 \mathrm{E}-5$ & $4.1 \mathrm{E}+4$ & $2.8 \mathrm{E}+4$ & 0.79 & $1.9 \mathrm{E}-5$ & $3.6 \mathrm{E}+4$ \\
\hline Bonvin $2013^{12}$ & sulfamethoxazole & sulfonamide photohydrolysis & 1.00 & $1.9 \mathrm{E}-5$ & $3.7 \mathrm{E}+4$ & $6.6 \mathrm{E}+4$ & 0.20 & $2.0 \mathrm{E}-6$ & $3.5 \mathrm{E}+5$ \\
\hline Bonvin 2013 & sulfamethoxazole & sulfonamide photohydrolysis & 1.00 & $1.9 \mathrm{E}-5$ & $3.7 \mathrm{E}+4$ & $6.6 \mathrm{E}+4$ & 0.02 & $2.0 \mathrm{E}-6$ & $3.5 \mathrm{E}+5$ \\
\hline Bonvin 2013 & sulfamethoxazole & sulfonamide S-C photohydrolysis & 1.00 & $1.9 \mathrm{E}-5$ & $3.7 \mathrm{E}+4$ & $6.6 \mathrm{E}+4$ & 0.11 & $8.4 \mathrm{E}-7$ & $8.2 \mathrm{E}+5$ \\
\hline Bonvin 2013 & sulfamethoxazole & sulfonamide S-C photohydrolysis & 1.00 & $1.9 \mathrm{E}-5$ & $3.7 \mathrm{E}+4$ & $6.6 \mathrm{E}+4$ & 0.05 & $8.4 \mathrm{E}-7$ & $8.2 \mathrm{E}+5$ \\
\hline Bonvin 2013 & $\begin{array}{l}\text { N-acetyl } \\
\text { sulfamethoxazole }\end{array}$ & sulfonamide photohydrolysis & 1.00 & $3.1 \mathrm{E}-6$ & $2.2 \mathrm{E}+5$ & $1.5 \mathrm{E}+5$ & 0.88 & $4.0 \mathrm{E}-6$ & $1.7 \mathrm{E}+5$ \\
\hline Bonvin 2013 & $\begin{array}{l}\text { N-acetyl } \\
\text { sulfamethoxazole }\end{array}$ & sulfonamide S-C photohydrolysis & 1.00 & $3.1 \mathrm{E}-6$ & $2.2 \mathrm{E}+5$ & $1.5 \mathrm{E}+5$ & 0.02 & $2.5 \mathrm{E}-7$ & $2.7 \mathrm{E}+6$ \\
\hline Bonvin 2013 & $\begin{array}{l}\text { N-acetyl } \\
\text { sulfamethoxazole }\end{array}$ & sulfonamide S-C photohydrolysis & 1.00 & $3.1 \mathrm{E}-6$ & $2.2 \mathrm{E}+5$ & $1.5 \mathrm{E}+5$ & 0.09 & $2.5 \mathrm{E}-7$ & $2.7 \mathrm{E}+6$ \\
\hline Bonvin 2013 & \begin{tabular}{|l|} 
4-nitroso \\
sulfamethoxazole
\end{tabular} & aromatic nitroso photoox & 1.00 & $1.8 \mathrm{E}-5$ & $3.9 \mathrm{E}+4$ & $3.9 \mathrm{E}+4$ & 0.55 & $9.9 \mathrm{E}-6$ & $7.0 \mathrm{E}+4$ \\
\hline
\end{tabular}




\begin{tabular}{|c|c|c|c|c|c|c|c|c|c|}
\hline reference & contaminant & reaction scheme & $\beta$ & $\begin{array}{l}k_{\mathrm{pa}} \\
\left(\mathrm{s}^{-1}\right)\end{array}$ & $\begin{array}{l}t_{1 / 2, \mathrm{pa}} \\
(\mathrm{s})\end{array}$ & $\begin{array}{l}t_{1 / 2, \text { pa,avg }} \\
(\mathrm{s})\end{array}$ & $\alpha$ & $\begin{array}{l}k_{\text {pr,avg }} \\
\left(\mathrm{s}^{-1}\right)\end{array}$ & $\begin{array}{l}t_{1 / 2, \text { pravg }} \\
(\mathrm{s})\end{array}$ \\
\hline Krieger $2000^{13}$ & florasulam & sulfonamide photohydrolysis & 1.00 & $2.2 \mathrm{E}-7$ & $3.1 \mathrm{E}+6$ & $3.1 \mathrm{E}+6$ & 0.71 & $1.6 \mathrm{E}-7$ & $4.4 \mathrm{E}+6$ \\
\hline Wilson $2000^{14}$ & metolachlor & $\mathrm{N}$-aryl amide photohydrolysis & 5.10 & $5.2 \mathrm{E}-7$ & $1.3 \mathrm{E}+6$ & $1.1 \mathrm{E}+6$ & 0.05 & $3.4 \mathrm{E}-8$ & $2.1 \mathrm{E}+7$ \\
\hline $\begin{array}{l}\text { McConville } \\
2016^{15}\end{array}$ & 5-chlorosalicylic acid & aromatic halide photohydrolysis & 3.33 & $7.6 \mathrm{E}-4$ & $9.1 \mathrm{E}+2$ & $9.1 \mathrm{E}+2$ & 0.41 & $3.1 \mathrm{E}-4$ & $2.2 \mathrm{E}+3$ \\
\hline $\begin{array}{l}\text { McConville } \\
2016\end{array}$ & gentisic acid & $\begin{array}{l}\text { aromatic carboxylic acid } \\
\text { photodecarboxylation to alcohol }\end{array}$ & 3.33 & $2.2 \mathrm{E}-5$ & $3.2 \mathrm{E}+4$ & $3.2 \mathrm{E}+4$ & 1.00 & $2.2 \mathrm{E}-5$ & $3.2 \mathrm{E}+4$ \\
\hline $\begin{array}{l}\text { McConville } \\
2016\end{array}$ & 2-chloro-4-nitroaniline & aromatic amine photohydrolysis & 3.33 & $2.2 \mathrm{E}-6$ & $3.2 \mathrm{E}+5$ & $3.2 \mathrm{E}+5$ & 0.05 & $9.9 \mathrm{E}-8$ & $7.0 \mathrm{E}+6$ \\
\hline $\begin{array}{l}\text { McConville } \\
2016\end{array}$ & 2-chloro-4-nitrophenol & aromatic halide photohydrolysis & 3.33 & $1.5 \mathrm{E}-6$ & $4.5 \mathrm{E}+5$ & $4.5 \mathrm{E}+5$ & 0.00 & $1.5 \mathrm{E}-9$ & $4.5 \mathrm{E}+8$ \\
\hline $\begin{array}{l}\text { McConville } \\
2016\end{array}$ & 4-nitrocatechol & aromatic nitro photohydrolysis & 3.33 & $8.9 \mathrm{E}-6$ & $7.8 \mathrm{E}+4$ & $7.8 \mathrm{E}+4$ & 0.55 & $4.9 \mathrm{E}-6$ & $1.4 \mathrm{E}+5$ \\
\hline Lam $2005^{16}$ & fluoxetine & trifluoromethyl photohydrolysis & 1.00 & $1.1 \mathrm{E}-6$ & $6.0 \mathrm{E}+5$ & $6.0 \mathrm{E}+5$ & 0.05 & $5.7 \mathrm{E}-8$ & $1.2 \mathrm{E}+7$ \\
\hline Lam 2005 & fluoxetine & aromatic ether photohydrolysis & 1.00 & $1.1 \mathrm{E}-6$ & $6.0 \mathrm{E}+5$ & $6.0 \mathrm{E}+5$ & 0.60 & $4.2 \mathrm{E}-7$ & $1.6 \mathrm{E}+6$ \\
\hline Tisler $2019^{17}$ & fluoxetine & aromatic ether photohydrolysis & & & & $6.0 \mathrm{E}+5$ & 0.31 & $4.2 \mathrm{E}-7$ & $1.6 \mathrm{E}+6$ \\
\hline Tisler 2019 & fluoxetine & aromatic ether photohydrolysis & & & & $6.0 \mathrm{E}+5$ & 0.19 & $4.2 \mathrm{E}-7$ & $1.6 \mathrm{E}+6$ \\
\hline Carey $1981^{18}$ & $\begin{array}{l}\text { 3-trifluoromethyl-4- } \\
\text { nitrophenol }\end{array}$ & aromatic nitro photohydrolysis & 0.08 & $3.2 \mathrm{E}-5$ & $2.2 \mathrm{E}+4$ & $2.9 \mathrm{E}+4$ & 0.55 & $1.3 \mathrm{E}-5$ & $5.3 \mathrm{E}+4$ \\
\hline Yamada $2010^{19}$ & fenthion & aromatic thioether photooxidation & & & & $6.5 \mathrm{E}+4$ & 0.79 & $4.9 \mathrm{E}-6$ & $1.4 \mathrm{E}+5$ \\
\hline Yamada 2010 & fenthion & $\begin{array}{l}\text { organophosphorus ester } \\
\text { photohydrolysis }\end{array}$ & & & & $6.5 \mathrm{E}+4$ & 0.01 & $3.1 \mathrm{E}-6$ & $2.3 \mathrm{E}+5$ \\
\hline Weber $2009^{20}$ & parathion-methyl & $\begin{array}{l}\text { organothiophosphorus ester } \\
\text { photooxidation to oxon }\end{array}$ & & & & $2.0 \mathrm{E}+6$ & 0.20 & $6.8 \mathrm{E}-8$ & $1.0 \mathrm{E}+7$ \\
\hline Weber 2009 & parathion-methyl & $\begin{array}{l}\text { organophosphorus ester } \\
\text { photohydrolysis }\end{array}$ & & & & $2.0 \mathrm{E}+6$ & 0.05 & $1.7 \mathrm{E}-8$ & $4.1 \mathrm{E}+7$ \\
\hline Weber 2009 & fenitrothion & $\begin{array}{l}\text { organothiophosphorus ester } \\
\text { photooxidation to oxon }\end{array}$ & & & & $3.5 \mathrm{E}+4$ & 0.20 & $2.1 \mathrm{E}-6$ & $3.3 \mathrm{E}+5$ \\
\hline Weber 2009 & fenitrothion & $\begin{array}{l}\text { organophosphorus ester } \\
\text { photohydrolysis }\end{array}$ & & & & $3.5 \mathrm{E}+4$ & 0.05 & $9.8 \mathrm{E}-7$ & $7.1 \mathrm{E}+5$ \\
\hline $\begin{array}{l}\text { Hirahara } \\
2003^{21}\end{array}$ & fenthion & $\begin{array}{l}\text { organophosphorus ester } \\
\text { photohydrolysis }\end{array}$ & & & & $6.5 \mathrm{E}+4$ & 0.40 & $3.1 \mathrm{E}-6$ & $2.3 \mathrm{E}+5$ \\
\hline
\end{tabular}




\begin{tabular}{|c|c|c|c|c|c|c|c|c|c|}
\hline reference & contaminant & reaction scheme & $\beta$ & $\begin{array}{l}k_{\mathrm{pa}} \\
\left(\mathrm{s}^{-1}\right)\end{array}$ & $\begin{array}{l}t_{1 / 2, \mathrm{pa}} \\
(\mathrm{s})\end{array}$ & $\begin{array}{l}t_{1 / 2, \text { pa,avg }} \\
(\mathrm{s})\end{array}$ & $\alpha$ & $\begin{array}{l}k_{\mathrm{pr}, \mathrm{avg}} \\
\left(\mathrm{s}^{-1}\right)\end{array}$ & $\begin{array}{l}t_{1 / 2, \text { pr,avg }} \\
\text { (s) }\end{array}$ \\
\hline Hirahara 2003 & fenthion & $\begin{array}{l}\text { organophosphorus ester } \\
\text { photohydrolysis }\end{array}$ & & & & $6.5 \mathrm{E}+4$ & 0.44 & $3.1 \mathrm{E}-6$ & $2.3 \mathrm{E}+5$ \\
\hline Hirahara 2003 & fenthion & aromatic thioether photooxidation & & & & $6.5 \mathrm{E}+4$ & 0.07 & $4.9 \mathrm{E}-6$ & $1.4 \mathrm{E}+5$ \\
\hline $\begin{array}{l}\text { Hirahara } \\
2001^{22}\end{array}$ & fenthion & aromatic thioether photooxidation & & & & $6.5 \mathrm{E}+4$ & 0.50 & $4.9 \mathrm{E}-6$ & $1.4 \mathrm{E}+5$ \\
\hline Katagi $1993^{23}$ & butamifos & $\begin{array}{l}\text { organothiophosphorus ester } \\
\text { photochemical oxygen transfer }\end{array}$ & 0.50 & $1.1 \mathrm{E}-3$ & $6.5 \mathrm{E}+2$ & $6.5 \mathrm{E}+2$ & 0.84 & $9.0 \mathrm{E}-4$ & $7.7 \mathrm{E}+2$ \\
\hline Katagi 1993 & butamifos & $\begin{array}{l}\text { organothiophosphorus ester } \\
\text { photooxidation to oxon }\end{array}$ & 0.50 & $1.1 \mathrm{E}-3$ & $6.5 \mathrm{E}+2$ & $6.5 \mathrm{E}+2$ & 0.02 & $2.6 \mathrm{E}-5$ & $2.7 \mathrm{E}+4$ \\
\hline Katagi 1993 & butamifos & $\begin{array}{l}\text { organophosphorus ester } \\
\text { photohydrolysis }\end{array}$ & 0.50 & $1.1 \mathrm{E}-3$ & $6.5 \mathrm{E}+2$ & $6.5 \mathrm{E}+2$ & 0.04 & $4.5 \mathrm{E}-5$ & $1.5 \mathrm{E}+4$ \\
\hline Mikami $1985^{24}$ & fenitrothion & $\begin{array}{l}\text { organothiophosphorus ester } \\
\text { photooxidation to oxon }\end{array}$ & 1.00 & $1.9 \mathrm{E}-5$ & $3.6 \mathrm{E}+4$ & $3.5 \mathrm{E}+4$ & 0.01 & $2.1 \mathrm{E}-6$ & $3.3 \mathrm{E}+5$ \\
\hline Mikami 1985 & fenitrothion & $\begin{array}{l}\text { aromatic methyl photooxidation } \\
\text { to carboxylic acid }\end{array}$ & 1.00 & $1.9 \mathrm{E}-5$ & $3.6 \mathrm{E}+4$ & $3.5 \mathrm{E}+4$ & 0.09 & $1.8 \mathrm{E}-6$ & $3.8 \mathrm{E}+5$ \\
\hline $\begin{array}{l}\text { Mansour } \\
1983^{25}\end{array}$ & parathion & $\begin{array}{l}\text { organothiophosphorus ester } \\
\text { photooxidation to oxon }\end{array}$ & & & & $1.7 \mathrm{E}+6$ & 0.11 & $4.3 \mathrm{E}-8$ & $1.6 \mathrm{E}+7$ \\
\hline Mansour 1983 & parathion & $\begin{array}{l}\text { organophosphorus ester } \\
\text { photohydrolysis }\end{array}$ & & & & $1.7 \mathrm{E}+6$ & 0.09 & $3.7 \mathrm{E}-8$ & $1.9 \mathrm{E}+7$ \\
\hline Ivie $1976^{26}$ & sulprofos & aromatic thioether photooxidation & 3.33 & $4.8 \mathrm{E}-6$ & $1.4 \mathrm{E}+5$ & $1.4 \mathrm{E}+5$ & 0.29 & $1.4 \mathrm{E}-6$ & $5.0 \mathrm{E}+5$ \\
\hline $\begin{array}{l}\text { Boulkamh } \\
2001^{27}\end{array}$ & metoxuron & aromatic halide photohydrolysis & 0.30 & $2.2 \mathrm{E}-6$ & $3.1 \mathrm{E}+5$ & $3.1 \mathrm{E}+5$ & 0.95 & $2.1 \mathrm{E}-6$ & $3.3 \mathrm{E}+5$ \\
\hline Tanaka $1986^{28}$ & diuron & $\begin{array}{l}\text { phenylurea photochemical N- } \\
\text { dealkylation }\end{array}$ & 1.00 & $3.2 \mathrm{E}-7$ & $2.2 \mathrm{E}+6$ & $2.3 \mathrm{E}+6$ & 0.04 & $1.2 \mathrm{E}-8$ & $5.9 \mathrm{E}+7$ \\
\hline Tanaka 1986 & diuron & $\begin{array}{l}\text { phenylurea N-methyl } \\
\text { photooxidation to N-formyl }\end{array}$ & 1.00 & $3.2 \mathrm{E}-7$ & $2.2 \mathrm{E}+6$ & $2.3 \mathrm{E}+6$ & 0.04 & $1.1 \mathrm{E}-8$ & $6.2 \mathrm{E}+7$ \\
\hline Tanaka 1986 & diuron & $\begin{array}{l}\text { aromatic } \\
\text { photohydrodehalogenation }\end{array}$ & 1.00 & $3.2 \mathrm{E}-7$ & $2.2 \mathrm{E}+6$ & $2.3 \mathrm{E}+6$ & 0.02 & 5.3E-9 & $1.3 \mathrm{E}+8$ \\
\hline Tanaka 1986 & diuron & aromatic halide photohydrolysis & 1.00 & $3.2 \mathrm{E}-7$ & $2.2 \mathrm{E}+6$ & $2.3 \mathrm{E}+6$ & 0.07 & $2.8 \mathrm{E}-8$ & $2.5 \mathrm{E}+7$ \\
\hline Tanaka 1986 & diuron & aromatic halide photohydrolysis & 1.00 & $3.2 \mathrm{E}-7$ & $2.2 \mathrm{E}+6$ & $2.3 \mathrm{E}+6$ & 0.12 & $2.8 \mathrm{E}-8$ & $2.5 \mathrm{E}+7$ \\
\hline Rosen $1969^{29}$ & linuron & aromatic halide photohydrolysis & 1.00 & $7.2 \mathrm{E}-8$ & $9.7 \mathrm{E}+6$ & $6.1 \mathrm{E}+6$ & 0.42 & $4.8 \mathrm{E}-8$ & $1.5 \mathrm{E}+7$ \\
\hline Rosen 1969 & linuron & $\begin{array}{l}\text { phenylurea photochemical N- } \\
\text { demethoxylation }\end{array}$ & 1.00 & $7.2 \mathrm{E}-8$ & $9.7 \mathrm{E}+6$ & $6.1 \mathrm{E}+6$ & 0.06 & 7.3E-9 & $9.5 \mathrm{E}+7$ \\
\hline
\end{tabular}




\begin{tabular}{|c|c|c|c|c|c|c|c|c|c|}
\hline reference & contaminant & reaction scheme & $\beta$ & $\begin{array}{l}k_{\mathrm{pa}} \\
\left(\mathrm{s}^{-1}\right)\end{array}$ & $\begin{array}{l}t_{1 / 2, \mathrm{pa}} \\
(\mathrm{s})\end{array}$ & $\begin{array}{l}t_{1 / 2, \text { pa,avg }} \\
(\mathrm{s})\end{array}$ & $\alpha$ & $\begin{array}{l}k_{\mathrm{pr}, \mathrm{avg}} \\
\left(\mathrm{s}^{-1}\right)\end{array}$ & $\begin{array}{l}t_{1 / 2, \text { pr,avg }} \\
(\mathrm{s})\end{array}$ \\
\hline Rosen 1968 & metobromuron & aromatic halide photohydrolysis & 1.00 & $1.5 \mathrm{E}-7$ & $4.6 \mathrm{E}+6$ & $4.6 \mathrm{E}+6$ & 0.75 & $1.1 \mathrm{E}-7$ & $6.2 \mathrm{E}+6$ \\
\hline Pirisi $1996^{30}$ & pirimicarb & $\begin{array}{l}\text { s-triazine side chain } \mathrm{N} \text {-alkyl } \\
\text { photooxidation to carbonyl }\end{array}$ & 0.30 & $4.9 \mathrm{E}-5$ & $1.4 \mathrm{E}+4$ & $1.4 \mathrm{E}+4$ & 0.32 & $1.5 \mathrm{E}-5$ & $4.5 \mathrm{E}+4$ \\
\hline Pirisi 1996 & pirimicarb & $\begin{array}{l}\text { aromatic carbamate } \\
\text { photohydrolysis }\end{array}$ & 0.30 & $4.9 \mathrm{E}-5$ & $1.4 \mathrm{E}+4$ & $1.4 \mathrm{E}+4$ & 0.10 & $4.7 \mathrm{E}-6$ & $1.5 \mathrm{E}+5$ \\
\hline Pirisi 1996 & pirimicarb & $\begin{array}{l}\text { s-triazine side chain } \\
\text { photochemical N-dealkylation }\end{array}$ & 0.30 & 4.9E-5 & $1.4 \mathrm{E}+4$ & $1.4 \mathrm{E}+4$ & 0.22 & $1.1 \mathrm{E}-5$ & $6.5 \mathrm{E}+4$ \\
\hline Temussi $2012^{31}$ & rivastigmine & $\begin{array}{l}\text { benzyl photodeamination to } \\
\text { carbonyl }\end{array}$ & 1.00 & $6.7 \mathrm{E}-7$ & $1.0 \mathrm{E}+6$ & $1.0 \mathrm{E}+6$ & 0.67 & $4.5 \mathrm{E}-7$ & $1.6 \mathrm{E}+6$ \\
\hline Temussi 2012 & rivastigmine & $\begin{array}{l}\text { benzyl photodeamination to } \\
\text { alcohol }\end{array}$ & 1.00 & $6.7 \mathrm{E}-7$ & $1.0 \mathrm{E}+6$ & $1.0 \mathrm{E}+6$ & 0.33 & $2.2 \mathrm{E}-7$ & $3.1 \mathrm{E}+6$ \\
\hline Ruzo $1985^{32}$ & thiobencarb & $\begin{array}{l}\text { benzyl thiocarbamate } \\
\text { photocleavage to carbonyl }\end{array}$ & & & & $1.9 \mathrm{E}+6$ & 0.14 & $5.1 \mathrm{E}-8$ & $1.4 \mathrm{E}+7$ \\
\hline Draper $1984^{33}$ & drepamon & $\begin{array}{l}\text { benzyl thio photooxidation to } \\
\text { sulfoxide }\end{array}$ & 1.00 & $5.3 \mathrm{E}-7$ & $1.3 \mathrm{E}+6$ & $1.3 \mathrm{E}+6$ & 0.15 & $8.0 \mathrm{E}-8$ & $8.6 \mathrm{E}+6$ \\
\hline Draper 1984 & drepamon sulfoxide & $\begin{array}{l}\text { benzyl thiocarbamate } \\
\text { photocleavage to carbonyl }\end{array}$ & 1.00 & $1.1 \mathrm{E}-6$ & $6.0 \mathrm{E}+5$ & $6.0 \mathrm{E}+5$ & 0.19 & $2.2 \mathrm{E}-7$ & $3.2 \mathrm{E}+6$ \\
\hline Vialaton $2000^{34}$ & ethiofencarb & $\begin{array}{l}\text { benzyl thio photooxidation to } \\
\text { sulfoxide }\end{array}$ & & & & $1.9 \mathrm{E}+6$ & 0.46 & $1.7 \mathrm{E}-7$ & $4.0 \mathrm{E}+6$ \\
\hline Miille $1983^{35}$ & 3,4-dichloroaniline & $\begin{array}{l}\text { aromatic } \\
\text { photohydrodehalogenation }\end{array}$ & & & & $8.0 \mathrm{E}+4$ & 0.02 & $1.7 \mathrm{E}-7$ & $4.0 \mathrm{E}+6$ \\
\hline $\begin{array}{l}\text { Machado } \\
1995^{36}\end{array}$ & bromoxynil & aromatic halide photohydrolysis & & & & $1.0 \mathrm{E}+4$ & 0.65 & $4.3 \mathrm{E}-5$ & $1.6 \mathrm{E}+4$ \\
\hline Miller $1979^{37}$ & 3,4-dichloroaniline & aromatic halide photohydrolysis & & & & $8.0 \mathrm{E}+4$ & 0.78 & $6.9 \mathrm{E}-6$ & $1.0 \mathrm{E}+5$ \\
\hline Miller 1979 & 3,4-dichloroaniline & $\begin{array}{l}\text { aromatic } \\
\text { photohydrodehalogenation }\end{array}$ & & & & $8.0 \mathrm{E}+4$ & 0.02 & $1.7 \mathrm{E}-7$ & $4.0 \mathrm{E}+6$ \\
\hline Carena $2018^{38}$ & 3,4-dichloroaniline & aromatic halide photohydrolysis & 1.00 & $9.5 \mathrm{E}-6$ & $7.3 \mathrm{E}+4$ & $8.0 \mathrm{E}+4$ & 0.80 & $6.9 \mathrm{E}-6$ & $1.0 \mathrm{E}+5$ \\
\hline Crosby $1973^{39}$ & $\begin{array}{l}2,4,5- \\
\text { trichlorophenoxyacetic } \\
\text { acid }\end{array}$ & aromatic ether photohydrolysis & 1.00 & $5.4 \mathrm{E}-7$ & $1.3 \mathrm{E}+6$ & $1.3 \mathrm{E}+6$ & 0.38 & $2.0 \mathrm{E}-7$ & $3.4 \mathrm{E}+6$ \\
\hline Crosby 1973 & $\begin{array}{l}2,4,5- \\
\text { trichlorophenoxyacetic } \\
\text { acid }\end{array}$ & aromatic halide photohydrolysis & 1.00 & $5.4 \mathrm{E}-7$ & $1.3 \mathrm{E}+6$ & $1.3 \mathrm{E}+6$ & 0.13 & $7.0 \mathrm{E}-8$ & $9.9 \mathrm{E}+6$ \\
\hline
\end{tabular}




\begin{tabular}{|c|c|c|c|c|c|c|c|c|c|}
\hline reference & contaminant & reaction scheme & $\beta$ & $\begin{array}{l}k_{\mathrm{pa}} \\
\left(\mathrm{s}^{-1}\right)\end{array}$ & $\begin{array}{l}t_{1 / 2, \mathrm{pa}} \\
(\mathrm{s})\end{array}$ & $\begin{array}{l}t_{1 / 2, \mathrm{pa}, \mathrm{avg}} \\
(\mathrm{s})\end{array}$ & $\alpha$ & $\begin{array}{l}k_{\mathrm{pr}, \mathrm{avg}} \\
\left(\mathrm{s}^{-1}\right)\end{array}$ & $\begin{array}{l}t_{1 / 2, \mathrm{pr}, \mathrm{avg}} \\
(\mathrm{s})\end{array}$ \\
\hline Meunier $2000^{40}$ & mecoprop & aromatic ether photohydrolysis & 1.00 & $1.0 \mathrm{E}-6$ & $6.9 \mathrm{E}+5$ & $6.9 \mathrm{E}+5$ & 0.10 & $1.0 \mathrm{E}-7$ & $6.8 \mathrm{E}+6$ \\
\hline Torrents $1997^{41}$ & atrazine & aromatic halide photohydrolysis & 3.00 & $3.7 \mathrm{E}-7$ & $1.9 \mathrm{E}+6$ & $2.4 \mathrm{E}+6$ & 0.58 & $1.7 \mathrm{E}-7$ & $4.2 \mathrm{E}+6$ \\
\hline Torrents 1997 & atrazine & $\begin{array}{l}\text { s-triazine side chain } \mathrm{N} \text {-alkyl } \\
\text { photooxidation to carbonyl }\end{array}$ & 3.00 & $3.7 \mathrm{E}-7$ & $1.9 \mathrm{E}+6$ & $2.4 \mathrm{E}+6$ & 0.17 & $4.8 \mathrm{E}-8$ & $1.5 \mathrm{E}+7$ \\
\hline Torrents 1997 & atrazine & $\begin{array}{l}\text { s-triazine side chain } \\
\text { photochemical N-dealkylation }\end{array}$ & 3.00 & $3.7 \mathrm{E}-7$ & $1.9 \mathrm{E}+6$ & $2.4 \mathrm{E}+6$ & 0.04 & $1.2 \mathrm{E}-8$ & $5.8 \mathrm{E}+7$ \\
\hline Torrents 1997 & atrazine & $\begin{array}{l}\text { s-triazine side chain } \mathrm{N} \text {-isopropyl } \\
\text { photooxidation to ketone }\end{array}$ & 3.00 & $3.7 \mathrm{E}-7$ & $1.9 \mathrm{E}+6$ & $2.4 \mathrm{E}+6$ & 0.17 & $4.8 \mathrm{E}-8$ & $1.5 \mathrm{E}+7$ \\
\hline Torrents 1997 & atrazine & $\begin{array}{l}\text { s-triazine side chain } \\
\text { photochemical N-dealkylation }\end{array}$ & 3.00 & $3.7 \mathrm{E}-7$ & $1.9 \mathrm{E}+6$ & $2.4 \mathrm{E}+6$ & 0.04 & $1.2 \mathrm{E}-8$ & $5.8 \mathrm{E}+7$ \\
\hline Torrents 1997 & desethyl atrazine & aromatic halide photohydrolysis & 3.00 & $3.7 \mathrm{E}-7$ & $1.9 \mathrm{E}+6$ & $1.9 \mathrm{E}+6$ & 0.42 & $1.5 \mathrm{E}-7$ & $4.5 \mathrm{E}+6$ \\
\hline Torrents 1997 & desethyl atrazine & $\begin{array}{l}\text { s-triazine side chain } \mathrm{N} \text {-isopropyl } \\
\text { photooxidation to ketone }\end{array}$ & 3.00 & $3.7 \mathrm{E}-7$ & $1.9 \mathrm{E}+6$ & $1.9 \mathrm{E}+6$ & 0.04 & $1.5 \mathrm{E}-8$ & $4.5 \mathrm{E}+7$ \\
\hline Torrents 1997 & desethyl atrazine & $\begin{array}{l}\text { s-triazine side chain } \\
\text { photochemical N-dealkylation }\end{array}$ & 3.00 & $3.7 \mathrm{E}-7$ & $1.9 \mathrm{E}+6$ & $1.9 \mathrm{E}+6$ & 0.21 & $7.7 \mathrm{E}-8$ & $9.0 \mathrm{E}+6$ \\
\hline Torrents 1997 & desisopropyl atrazine & aromatic halide photohydrolysis & 3.00 & $3.7 \mathrm{E}-7$ & $1.9 \mathrm{E}+6$ & $1.9 \mathrm{E}+6$ & 0.21 & 7.7E-8 & $9.0 \mathrm{E}+6$ \\
\hline Torrents 1997 & desisopropyl atrazine & $\begin{array}{l}\text { s-triazine side chain } \mathrm{N} \text {-alkyl } \\
\text { photooxidation to carbonyl }\end{array}$ & 3.00 & $3.7 \mathrm{E}-7$ & $1.9 \mathrm{E}+6$ & $1.9 \mathrm{E}+6$ & 0.04 & $1.5 \mathrm{E}-8$ & $4.5 \mathrm{E}+7$ \\
\hline Torrents 1997 & desisopropyl atrazine & $\begin{array}{l}\text { s-triazine side chain } \\
\text { photochemical N-dealkylation }\end{array}$ & 3.00 & $3.7 \mathrm{E}-7$ & $1.9 \mathrm{E}+6$ & $1.9 \mathrm{E}+6$ & 0.21 & $7.7 \mathrm{E}-8$ & $9.0 \mathrm{E}+6$ \\
\hline Ruggeri $2013^{42}$ & ibuprofen & $\begin{array}{l}\text { aromatic acetic acid } \\
\text { photodecarboxylation to carbonyl }\end{array}$ & & & & $1.2 \mathrm{E}+7$ & 0.25 & $1.4 \mathrm{E}-8$ & $4.9 \mathrm{E}+7$ \\
\hline Poiger $2001^{43}$ & diclofenac & $\begin{array}{l}\text { diphenylamine dehalogenative } \\
\text { photocyclization to carbazole }\end{array}$ & 3.33 & $2.2 \mathrm{E}-4$ & $3.2 \mathrm{E}+3$ & $2.1 \mathrm{E}+4$ & 0.10 & $1.0 \mathrm{E}-5$ & $6.9 \mathrm{E}+4$ \\
\hline $\begin{array}{l}\text { Eriksson } \\
2010^{44} \\
\end{array}$ & diclofenac & $\begin{array}{l}\text { diphenylamine dehalogenative } \\
\text { photocyclization to carbazole }\end{array}$ & & & & $2.1 \mathrm{E}+4$ & 0.50 & $1.0 \mathrm{E}-5$ & $6.9 \mathrm{E}+4$ \\
\hline Kotnik $2016^{45}$ & ketoprofen & $\begin{array}{l}\text { aromatic acetic acid } \\
\text { photodecarboxylation to carbonyl }\end{array}$ & 1.67 & $2.5 \mathrm{E}-4$ & $2.8 \mathrm{E}+3$ & $2.6 \mathrm{E}+3$ & 0.70 & $1.9 \mathrm{E}-4$ & $3.7 \mathrm{E}+3$ \\
\hline Kotnik 2016 & ketoprofen & $\begin{array}{l}\text { aromatic acetic acid } \\
\text { photodecarboxylation }\end{array}$ & 1.67 & $2.5 \mathrm{E}-4$ & $2.8 \mathrm{E}+3$ & $2.6 \mathrm{E}+3$ & 0.30 & $8.0 \mathrm{E}-5$ & $8.7 \mathrm{E}+3$ \\
\hline Packer $2003^{46}$ & naproxen & $\begin{array}{l}\text { aromatic acetic acid } \\
\text { photodecarboxylation to carbonyl }\end{array}$ & 3.33 & $1.0 \mathrm{E}-4$ & $6.7 \mathrm{E}+3$ & $2.5 \mathrm{E}+4$ & 0.27 & $9.7 \mathrm{E}-6$ & $7.1 \mathrm{E}+4$ \\
\hline
\end{tabular}




\begin{tabular}{|c|c|c|c|c|c|c|c|c|c|}
\hline reference & contaminant & reaction scheme & $\beta$ & $\begin{array}{l}k_{\mathrm{pa}} \\
\left(\mathrm{s}^{-1}\right)\end{array}$ & $\begin{array}{l}t_{1 / 2, \mathrm{pa}} \\
(\mathrm{s})\end{array}$ & $\begin{array}{l}t_{1 / 2, \text { pa,avg }} \\
\text { (s) }\end{array}$ & $\alpha$ & \begin{tabular}{|l}
$\begin{array}{l}k_{\mathrm{pr}, \mathrm{avg}} \\
\left(\mathrm{s}^{-1}\right)\end{array}$ \\
\end{tabular} & $\begin{array}{l}t_{1 / 2, \text { pravg }} \\
\text { (s) }\end{array}$ \\
\hline $\begin{array}{l}\text { DellaGreca } \\
2004^{47}\end{array}$ & naproxen & $\begin{array}{l}\text { aromatic acetic acid } \\
\text { photodecarboxylation }\end{array}$ & & & & $2.5 \mathrm{E}+4$ & 0.06 & $1.7 \mathrm{E}-6$ & $4.1 \mathrm{E}+5$ \\
\hline $\begin{array}{l}\text { DellaGreca } \\
2004\end{array}$ & naproxen & $\begin{array}{l}\text { aromatic acetic acid } \\
\text { photodecarboxylation to alcohol }\end{array}$ & & & & $2.5 \mathrm{E}+4$ & 0.18 & $5.1 \mathrm{E}-6$ & $1.4 \mathrm{E}+5$ \\
\hline $\begin{array}{l}\text { DellaGreca } \\
2004\end{array}$ & naproxen & $\begin{array}{l}\text { aromatic acetic acid } \\
\text { photodecarboxylation to carbonyl }\end{array}$ & & & & $2.5 \mathrm{E}+4$ & 0.42 & $9.7 \mathrm{E}-6$ & $7.1 \mathrm{E}+4$ \\
\hline Temussi $2011^{48}$ & indomethacin & $\begin{array}{l}\text { aromatic acetic acid } \\
\text { photodecarboxylation to carbonyl }\end{array}$ & 1.67 & $9.3 \mathrm{E}-6$ & $7.4 \mathrm{E}+4$ & $7.4 \mathrm{E}+4$ & 0.34 & $3.2 \mathrm{E}-6$ & $2.2 \mathrm{E}+5$ \\
\hline Temussi 2011 & indomethacin & $\begin{array}{l}\text { aromatic acetic acid } \\
\text { photodecarboxylation }\end{array}$ & 1.67 & $9.3 \mathrm{E}-6$ & $7.4 \mathrm{E}+4$ & $7.4 \mathrm{E}+4$ & 0.06 & $5.6 \mathrm{E}-7$ & $1.2 \mathrm{E}+6$ \\
\hline $\begin{array}{l}\text { Wammer } \\
2016^{49}\end{array}$ & altrenogest & altrenogest photocycloaddition & 1.67 & $1.5 \mathrm{E}-2$ & $4.5 \mathrm{E}+1$ & $4.5 \mathrm{E}+1$ & 1.00 & $1.5 \mathrm{E}-2$ & $4.5 \mathrm{E}+1$ \\
\hline $\begin{array}{l}\text { Kolodziej } \\
2013^{50}\end{array}$ & trenbolone & $\begin{array}{l}\text { trienone steroid photohydration } \\
\text { (C12) }\end{array}$ & & & & $5.6 \mathrm{E}+3$ & 0.16 & $2.0 \mathrm{E}-5$ & $3.5 \mathrm{E}+4$ \\
\hline Kolodziej 2013 & trenbolone & $\begin{array}{l}\text { trienone steroid photooxidation to } \\
\text { dialdehyde }\end{array}$ & & & & $5.6 \mathrm{E}+3$ & 0.07 & $8.7 \mathrm{E}-6$ & $8.0 \mathrm{E}+4$ \\
\hline Pflug $2017^{51}$ & dienogest & $\begin{array}{l}\text { dienone steroid photohydration } \\
\text { (C9) }\end{array}$ & 3.33 & $3.7 \mathrm{E}-3$ & $1.9 \mathrm{E}+2$ & $1.9 \mathrm{E}+2$ & 0.70 & $2.0 \mathrm{E}-3$ & $3.5 \mathrm{E}+2$ \\
\hline Pflug 2017 & dienogest & $\begin{array}{l}\text { dienone steroid photohydration } \\
\text { (C9) }\end{array}$ & 3.33 & $3.7 \mathrm{E}-3$ & $1.9 \mathrm{E}+2$ & $1.9 \mathrm{E}+2$ & 0.10 & $2.0 \mathrm{E}-3$ & $3.5 \mathrm{E}+2$ \\
\hline Pflug 2017 & dienogest & $\begin{array}{l}\text { dienone steroid photohydration } \\
\text { (C9) }\end{array}$ & 3.33 & $3.7 \mathrm{E}-3$ & $1.9 \mathrm{E}+2$ & $1.9 \mathrm{E}+2$ & 0.80 & $2.0 \mathrm{E}-3$ & $3.5 \mathrm{E}+2$ \\
\hline $\begin{array}{l}\text { Kliegman } \\
2013^{52}\end{array}$ & triclosan & $\begin{array}{l}\text { phenoxyphenol dehalogenative } \\
\text { photocyclization to dioxin }\end{array}$ & & & & $4.0 \mathrm{E}+4$ & 0.04 & $7.3 \mathrm{E}-7$ & $9.5 \mathrm{E}+5$ \\
\hline Kliegman 2013 & triclosan & $\begin{array}{l}\text { phenoxyphenol dehalogenative } \\
\text { photorearrangement }\end{array}$ & & & & $4.0 \mathrm{E}+4$ & 0.10 & $1.8 \mathrm{E}-6$ & $4.0 \mathrm{E}+5$ \\
\hline Kliegman 2013 & triclosan & $\begin{array}{l}\text { aromatic } \\
\text { photohydrodehalogenation }\end{array}$ & & & & $4.0 \mathrm{E}+4$ & 0.01 & $8.8 \mathrm{E}-8$ & $7.9 \mathrm{E}+6$ \\
\hline Kliegman 2013 & triclosan & $\begin{array}{l}\text { phenoxyphenol ether } \\
\text { photocleavage }\end{array}$ & & & & $4.0 \mathrm{E}+4$ & 0.07 & $1.5 \mathrm{E}-6$ & $4.7 \mathrm{E}+5$ \\
\hline Buth $2009^{53}$ & triclosan & $\begin{array}{l}\text { phenoxyphenol dehalogenative } \\
\text { photocyclization to dioxin }\end{array}$ & & & & $4.0 \mathrm{E}+4$ & 0.04 & 7.3E-7 & $9.5 \mathrm{E}+5$ \\
\hline Buth 2009 & triclosan & $\begin{array}{l}\text { phenoxyphenol ether } \\
\text { photocleavage }\end{array}$ & & & & $4.0 \mathrm{E}+4$ & 0.10 & $1.5 \mathrm{E}-6$ & $4.7 \mathrm{E}+5$ \\
\hline
\end{tabular}




\begin{tabular}{|c|c|c|c|c|c|c|c|c|c|}
\hline reference & contaminant & reaction scheme & $\beta$ & $\begin{array}{l}k_{\mathrm{pa}} \\
\left(\mathrm{s}^{-1}\right)\end{array}$ & $\begin{array}{l}t_{1 / 2, \mathrm{pa}} \\
(\mathrm{s})\end{array}$ & $\begin{array}{l}t_{1 / 2, \text { pa,avg }} \\
(\mathrm{s})\end{array}$ & $\alpha$ & $\begin{array}{l}k_{\mathrm{pr}, \text { avg }} \\
\left(\mathrm{s}^{-1}\right)\end{array}$ & $\begin{array}{l}t_{1 / 2, \text { pr,avg }} \\
(\mathrm{s})\end{array}$ \\
\hline Latch $2003^{54}$ & triclosan & $\begin{array}{l}\text { phenoxyphenol dehalogenative } \\
\text { photocyclization to dioxin }\end{array}$ & & & & $4.0 \mathrm{E}+4$ & 0.07 & 7.3E-7 & $9.5 \mathrm{E}+5$ \\
\hline Steen $2009^{55}$ & 6-OH PBDE 47 & $\begin{array}{l}\text { phenoxyphenol dehalogenative } \\
\text { photocyclization to dioxin }\end{array}$ & 3.33 & $1.7 \mathrm{E}-4$ & $4.1 \mathrm{E}+3$ & $4.1 \mathrm{E}+3$ & 0.04 & $5.9 \mathrm{E}-6$ & $1.2 \mathrm{E}+5$ \\
\hline Steen 2009 & 3-Cl-6-OH PBDE 47 & $\begin{array}{l}\text { phenoxyphenol dehalogenative } \\
\text { photocyclization to dioxin }\end{array}$ & 3.33 & $4.2 \mathrm{E}-4$ & $1.7 \mathrm{E}+3$ & $1.7 \mathrm{E}+3$ & 0.01 & $5.5 \mathrm{E}-6$ & $1.3 \mathrm{E}+5$ \\
\hline Steen 2009 & 5-Cl-6-OH PBDE 47 & $\begin{array}{l}\text { phenoxyphenol dehalogenative } \\
\text { photocyclization to dioxin }\end{array}$ & 3.33 & $2.6 \mathrm{E}-4$ & $2.6 \mathrm{E}+3$ & $2.6 \mathrm{E}+3$ & 0.02 & $5.5 \mathrm{E}-6$ & $1.3 \mathrm{E}+5$ \\
\hline Steen 2009 & 3,5-Cl-6-OH PBDE 47 & $\begin{array}{l}\text { phenoxyphenol dehalogenative } \\
\text { photocyclization to dioxin }\end{array}$ & 3.33 & $3.6 \mathrm{E}-4$ & $1.9 \mathrm{E}+3$ & $1.9 \mathrm{E}+3$ & 0.01 & $4.0 \mathrm{E}-6$ & $1.8 \mathrm{E}+5$ \\
\hline Zhang $2016^{56}$ & triclosan & $\begin{array}{l}\text { phenoxyphenol dehalogenative } \\
\text { photocyclization to dioxin }\end{array}$ & & & & $4.0 \mathrm{E}+4$ & 0.02 & 7.3E-7 & $9.5 \mathrm{E}+5$ \\
\hline $\operatorname{Kim} 2000^{57}$ & $\begin{array}{l}1,2,7,8- \\
\text { tetrachlorodibenzo-p- } \\
\text { dioxin }\end{array}$ & $\begin{array}{l}\text { aromatic } \\
\text { photohydrodehalogenation }\end{array}$ & 1.67 & $1.8 \mathrm{E}-5$ & $3.8 \mathrm{E}+4$ & $3.8 \mathrm{E}+4$ & 0.03 & $5.4 \mathrm{E}-7$ & $1.3 \mathrm{E}+6$ \\
\hline Kim 2000 & $\begin{array}{l}\text { octachlorodibenzo-p- } \\
\text { dioxin }\end{array}$ & $\begin{array}{l}\text { aromatic } \\
\text { photohydrodehalogenation }\end{array}$ & 1.67 & $5.0 \mathrm{E}-6$ & $1.4 \mathrm{E}+5$ & $1.4 \mathrm{E}+5$ & 0.02 & $1.0 \mathrm{E}-7$ & $6.9 \mathrm{E}+6$ \\
\hline Kim 2000 & $\begin{array}{l}1,2,7,8- \\
\text { tetrachlorodibenzofuran }\end{array}$ & $\begin{array}{l}\text { aromatic } \\
\text { photohydrodehalogenation }\end{array}$ & 1.67 & $1.4 \mathrm{E}-5$ & $5.0 \mathrm{E}+4$ & $5.0 \mathrm{E}+4$ & 0.03 & $4.1 \mathrm{E}-7$ & $1.7 \mathrm{E}+6$ \\
\hline Kim 2000 & octachlorodibenzofuran & $\begin{array}{l}\text { aromatic } \\
\text { photohydrodehalogenation }\end{array}$ & 1.67 & $1.8 \mathrm{E}-5$ & $3.9 \mathrm{E}+4$ & $3.9 \mathrm{E}+4$ & 0.02 & $3.6 \mathrm{E}-7$ & $2.0 \mathrm{E}+6$ \\
\hline Dung $1994^{58}$ & $\begin{array}{l}\text { 2,7- } \\
\text { dichlorodibenzofuran }\end{array}$ & $\begin{array}{l}\text { aromatic } \\
\text { photohydrodehalogenation }\end{array}$ & 1.67 & $7.0 \mathrm{E}-6$ & $9.9 \mathrm{E}+4$ & $9.9 \mathrm{E}+4$ & 0.01 & $7.0 \mathrm{E}-8$ & $9.9 \mathrm{E}+6$ \\
\hline Dung 1994 & $\begin{array}{l}2,3,7,8- \\
\text { tetrachlorodibenzofuran }\end{array}$ & $\begin{array}{l}\text { aromatic } \\
\text { photohydrodehalogenation }\end{array}$ & 1.67 & $1.1 \mathrm{E}-5$ & $6.6 \mathrm{E}+4$ & $6.6 \mathrm{E}+4$ & 0.01 & $1.1 \mathrm{E}-7$ & $6.6 \mathrm{E}+6$ \\
\hline Vialaton $2002^{59}$ & ethiofencarb & $\begin{array}{l}\text { benzyl thio photooxidation to } \\
\text { sulfoxide }\end{array}$ & 1.67 & $3.6 \mathrm{E}-7$ & $1.9 \mathrm{E}+6$ & $1.9 \mathrm{E}+6$ & 0.50 & $1.7 \mathrm{E}-7$ & $4.0 \mathrm{E}+6$ \\
\hline Vialaton $2001^{60}$ & acifluorfen & $\begin{array}{l}\text { aromatic carboxylic acid } \\
\text { photodecarboxylation }\end{array}$ & 1.00 & $8.0 \mathrm{E}-7$ & $8.6 \mathrm{E}+5$ & $8.3 \mathrm{E}+5$ & 0.40 & $3.3 \mathrm{E}-7$ & $2.1 \mathrm{E}+6$ \\
\hline Aguer $1998^{61}$ & napropamide & aromatic ether photohydrolysis & 3.33 & $1.7 \mathrm{E}-4$ & $4.0 \mathrm{E}+3$ & $2.7 \mathrm{E}+3$ & 0.10 & $2.6 \mathrm{E}-5$ & $2.7 \mathrm{E}+4$ \\
\hline Aguer 1998 & napropamide & $\begin{array}{l}\text { 1-naphthoxy photorearrangement } \\
\text { (C2) }\end{array}$ & 3.33 & $1.7 \mathrm{E}-4$ & $4.0 \mathrm{E}+3$ & $2.7 \mathrm{E}+3$ & 0.60 & $1.1 \mathrm{E}-4$ & $6.1 \mathrm{E}+3$ \\
\hline Aguer 1998 & napropamide & $\begin{array}{l}\text { 1-naphthoxy photorearrangement } \\
\text { (C4) }\end{array}$ & 3.33 & $1.7 \mathrm{E}-4$ & $4.0 \mathrm{E}+3$ & $2.7 \mathrm{E}+3$ & 0.15 & $6.8 \mathrm{E}-5$ & $1.0 \mathrm{E}+4$ \\
\hline
\end{tabular}




\begin{tabular}{|c|c|c|c|c|c|c|c|c|c|}
\hline reference & contaminant & reaction scheme & $\beta$ & $\begin{array}{l}k_{\mathrm{pa}} \\
\left(\mathrm{s}^{-1}\right)\end{array}$ & $\begin{array}{l}t_{1 / 2, \mathrm{pa}} \\
(\mathrm{s})\end{array}$ & $\begin{array}{l}t_{1 / 2, \text { pa,avg }} \\
(\mathrm{s})\end{array}$ & $\alpha$ & $\begin{array}{l}k_{\mathrm{pr}, \mathrm{avg}} \\
\left(\mathrm{s}^{-1}\right)\end{array}$ & $\begin{array}{l}t_{1 / 2, \text { pr,avg }} \\
(\mathrm{s})\end{array}$ \\
\hline Chang $1991^{62}$ & napropamide & $\begin{array}{l}\text { 1-naphthoxy photorearrangement } \\
\text { (C2) }\end{array}$ & 4.00 & $5.1 \mathrm{E}-4$ & $1.4 \mathrm{E}+3$ & $2.7 \mathrm{E}+3$ & 0.28 & $1.1 \mathrm{E}-4$ & $6.1 \mathrm{E}+3$ \\
\hline Chang 1991 & napropamide & $\begin{array}{l}\text { 1-naphthoxy photorearrangement } \\
\text { (C4) }\end{array}$ & 4.00 & $5.1 \mathrm{E}-4$ & $1.4 \mathrm{E}+3$ & $2.7 \mathrm{E}+3$ & 0.38 & $6.8 \mathrm{E}-5$ & $1.0 \mathrm{E}+4$ \\
\hline $\begin{array}{l}\text { Oyamada } \\
1996^{63}\end{array}$ & naproanilide & $\mathrm{N}$-aryl amide photohydrolysis & 1.67 & $6.9 \mathrm{E}-4$ & $1.0 \mathrm{E}+3$ & $1.0 \mathrm{E}+3$ & 0.00 & $2.1 \mathrm{E}-6$ & $3.3 \mathrm{E}+5$ \\
\hline Oyamada 1996 & naproanilide & aromatic ether photohydrolysis & 1.67 & $6.9 \mathrm{E}-4$ & $1.0 \mathrm{E}+3$ & $1.0 \mathrm{E}+3$ & 0.04 & $3.0 \mathrm{E}-5$ & $2.3 \mathrm{E}+4$ \\
\hline Oyamada 1996 & $\begin{array}{l}\text { 2-(2- } \\
\text { naphthoxy)propionic } \\
\text { acid }\end{array}$ & aromatic ether photohydrolysis & 1.67 & $5.8 \mathrm{E}-5$ & $1.2 \mathrm{E}+4$ & $1.2 \mathrm{E}+4$ & 0.01 & $6.9 \mathrm{E}-7$ & $1.0 \mathrm{E}+6$ \\
\hline Adachi $2018^{64}$ & mandestrobin & $\begin{array}{l}\text { benzyl phenyl ether } \\
\text { photorearrangement (o) }\end{array}$ & 1.69 & $1.8 \mathrm{E}-6$ & $4.0 \mathrm{E}+5$ & $4.0 \mathrm{E}+5$ & 0.27 & $4.8 \mathrm{E}-7$ & $1.5 \mathrm{E}+6$ \\
\hline Adachi 2018 & mandestrobin & $\begin{array}{l}\text { benzyl phenyl ether } \\
\text { photorearrangement }(\mathrm{p})\end{array}$ & 1.69 & $1.8 \mathrm{E}-6$ & $4.0 \mathrm{E}+5$ & $4.0 \mathrm{E}+5$ & 0.22 & $3.9 \mathrm{E}-7$ & $1.8 \mathrm{E}+6$ \\
\hline Adachi 2018 & mandestrobin & aromatic ether photohydrolysis & 1.69 & $1.8 \mathrm{E}-6$ & $4.0 \mathrm{E}+5$ & $4.0 \mathrm{E}+5$ & 0.12 & $2.1 \mathrm{E}-7$ & $3.3 \mathrm{E}+6$ \\
\hline $\begin{array}{l}\text { Wei-haas } \\
2015^{65}\end{array}$ & BDE 47 & $\begin{array}{l}\text { aromatic } \\
\text { photohydrodehalogenation }\end{array}$ & 3.60 & $6.9 \mathrm{E}-7$ & $1.0 \mathrm{E}+6$ & $1.0 \mathrm{E}+6$ & 0.10 & $6.6 \mathrm{E}-8$ & $1.1 \mathrm{E}+7$ \\
\hline Halasz $2018^{66}$ & nitroguanidine & $\begin{array}{l}\text { nitroguanidine photochemical N- } \\
\text { denitration }\end{array}$ & 3.93 & $3.5 \mathrm{E}-6$ & $2.0 \mathrm{E}+5$ & $1.3 \mathrm{E}+5$ & 0.94 & $5.2 \mathrm{E}-6$ & $1.3 \mathrm{E}+5$ \\
\hline Haag $1990^{67}$ & nitroguanidine & $\begin{array}{l}\text { nitroguanidine nitro } \\
\text { photohydrolysis }\end{array}$ & 1.00 & $1.3 \mathrm{E}-5$ & $5.2 \mathrm{E}+4$ & $1.3 \mathrm{E}+5$ & 0.40 & $2.2 \mathrm{E}-6$ & $3.1 \mathrm{E}+5$ \\
\hline Rao $2013^{68}$ & 2,4-dinitroanisole & aromatic ether photohydrolysis & 2.11 & $4.7 \mathrm{E}-6$ & $1.5 \mathrm{E}+5$ & $7.1 \mathrm{E}+5$ & 0.03 & $2.9 \mathrm{E}-8$ & $2.4 \mathrm{E}+7$ \\
\hline Vione $2016^{69}$ & nitrobenzene & aromatic nitro photohydrolysis & 1.00 & $3.6 \mathrm{E}-6$ & $1.9 \mathrm{E}+5$ & $4.6 \mathrm{E}+5$ & 0.01 & $2.1 \mathrm{E}-8$ & $3.3 \mathrm{E}+7$ \\
\hline McFall $2016^{70}$ & 2-nitrobenzaldehyde & $\begin{array}{l}\text { 2-nitrobenzaldehyde } \\
\text { photorearrangement }\end{array}$ & & & & $1.3 \mathrm{E}+3$ & 1.00 & $5.4 \mathrm{E}-4$ & $1.3 \mathrm{E}+3$ \\
\hline Plumlee $2007^{71}$ & $\begin{array}{l}\mathrm{N}- \\
\text { nitrosodimethylamine }\end{array}$ & nitrosamine $\mathrm{N}-\mathrm{N}$ photocleavage & 5.10 & $1.4 \mathrm{E}-4$ & $4.9 \mathrm{E}+3$ & $4.9 \mathrm{E}+3$ & 0.19 & $2.7 \mathrm{E}-5$ & $2.6 \mathrm{E}+4$ \\
\hline Plumlee 2007 & $\begin{array}{l}\mathrm{N}- \\
\text { nitrosodimethylamine }\end{array}$ & nitrosamine $\mathrm{N}-\mathrm{C}$ photocleavage & 5.10 & $1.4 \mathrm{E}-4$ & $4.9 \mathrm{E}+3$ & $4.9 \mathrm{E}+3$ & 0.39 & $5.5 \mathrm{E}-5$ & $1.3 \mathrm{E}+4$ \\
\hline $\begin{array}{l}\text { Williams } \\
2018^{72}\end{array}$ & $\begin{array}{l}\text { benzobicyclon } \\
\text { hydrolysate }\end{array}$ & $\begin{array}{l}\text { beta-triketone dehalogenative } \\
\text { photocyclization to pyran }\end{array}$ & 1.00 & $6.0 \mathrm{E}-7$ & $1.2 \mathrm{E}+6$ & $1.2 \mathrm{E}+6$ & 0.06 & $3.5 \mathrm{E}-8$ & $2.0 \mathrm{E}+7$ \\
\hline Williams 2018 & $\begin{array}{l}\text { benzobicyclon } \\
\text { hydrolysate }\end{array}$ & $\begin{array}{l}\text { beta-triketone alpha } \\
\text { photocleavage to carboxylic acid }\end{array}$ & 1.00 & $6.0 \mathrm{E}-7$ & $1.2 \mathrm{E}+6$ & $1.2 \mathrm{E}+6$ & 0.08 & $4.7 \mathrm{E}-8$ & $1.5 \mathrm{E}+7$ \\
\hline
\end{tabular}




\begin{tabular}{|c|c|c|c|c|c|c|c|c|c|}
\hline reference & contaminant & reaction scheme & $\beta$ & $\begin{array}{l}k_{\mathrm{pa}} \\
\left(\mathrm{s}^{-1}\right)\end{array}$ & $\begin{array}{l}t_{1 / 2, \mathrm{pa}} \\
(\mathrm{s})\end{array}$ & $\begin{array}{l}t_{1 / 2, \text { pa,avg }} \\
\text { (s) }\end{array}$ & $\alpha$ & $\begin{array}{l}k_{\mathrm{pr}, \mathrm{avg}} \\
\left(\mathrm{s}^{-1}\right)\end{array}$ & $\begin{array}{l}t_{1 / 2, \text { pr,avg }} \\
(\mathrm{s})\end{array}$ \\
\hline $\begin{array}{l}\text { Nishiyama } \\
2010^{73}\end{array}$ & metofluthrin & $\begin{array}{l}\text { pyrethroid carboxylic acid ester } \\
\text { photohydrolysis }\end{array}$ & 1.56 & $2.3 \mathrm{E}-6$ & $3.0 \mathrm{E}+5$ & $3.0 \mathrm{E}+5$ & 0.20 & $4.6 \mathrm{E}-7$ & $1.5 \mathrm{E}+6$ \\
\hline Katagi $1992^{74}$ & 3-phenoxybenzoic acid & diphenyl ether photohydrolysis & 1.00 & $2.7 \mathrm{E}-6$ & $2.6 \mathrm{E}+5$ & $2.6 \mathrm{E}+5$ & 0.04 & $1.1 \mathrm{E}-7$ & $6.1 \mathrm{E}+6$ \\
\hline $\begin{array}{l}\text { Wamhoff } \\
1999^{75}\end{array}$ & imidacloprid & $\begin{array}{l}\text { nitroguanidine imine } \\
\text { photohydrolysis }\end{array}$ & & & & $9.0 \mathrm{E}+3$ & 0.03 & $2.5 \mathrm{E}-6$ & $2.8 \mathrm{E}+5$ \\
\hline $\begin{array}{l}\text { Mulligan } \\
2015^{76}\end{array}$ & clothianidin & $\begin{array}{l}\text { nitroguanidine imine } \\
\text { photohydrolysis }\end{array}$ & & & & $1.8 \mathrm{E}+4$ & 0.17 & $6.5 \mathrm{E}-6$ & $1.1 \mathrm{E}+5$ \\
\hline $\begin{array}{l}\text { De Laurentiis } \\
2012^{77}\end{array}$ & carbamazepine & carbamazepine photoepoxidation & 1.00 & $8.1 \mathrm{E}-7$ & $8.6 \mathrm{E}+5$ & $1.1 \mathrm{E}+6$ & 0.02 & $8.3 \mathrm{E}-8$ & $8.3 \mathrm{E}+6$ \\
\hline Lam $2005^{78}$ & carbamazepine & carbamazepine photoepoxidation & 5.10 & $3.3 \mathrm{E}-7$ & $2.1 \mathrm{E}+6$ & $1.1 \mathrm{E}+6$ & 0.25 & $8.3 \mathrm{E}-8$ & $8.3 \mathrm{E}+6$ \\
\hline West $2012^{79}$ & diazepam & diazepam ring photocleavage & 3.33 & $5.6 \mathrm{E}-7$ & $1.2 \mathrm{E}+6$ & $9.1 \mathrm{E}+5$ & 0.77 & $5.9 \mathrm{E}-7$ & $1.2 \mathrm{E}+6$ \\
\hline West 2012 & nordiazepam & diazepam ring photocleavage & 3.33 & $3.0 \mathrm{E}-7$ & $2.3 \mathrm{E}+6$ & $2.3 \mathrm{E}+6$ & 1.00 & $3.0 \mathrm{E}-7$ & $2.3 \mathrm{E}+6$ \\
\hline Kramer $1996^{80}$ & diaminostilbene DAS 1 & diarylethene photooxidation & 3.33 & $1.1 \mathrm{E}-5$ & $6.5 \mathrm{E}+4$ & $6.5 \mathrm{E}+4$ & 0.09 & $9.6 \mathrm{E}-7$ & $7.2 \mathrm{E}+5$ \\
\hline Kramer 1996 & diaminostilbene DAS 1 & diarylethene photooxidation & 3.33 & $1.1 \mathrm{E}-5$ & $6.5 \mathrm{E}+4$ & $6.5 \mathrm{E}+4$ & 0.09 & $9.6 \mathrm{E}-7$ & $7.2 \mathrm{E}+5$ \\
\hline Kramer 1996 & diaminostilbene DAS 1 & diarylethene photohydration & 3.33 & $1.1 \mathrm{E}-5$ & $6.5 \mathrm{E}+4$ & $6.5 \mathrm{E}+4$ & 0.65 & 7.0E-6 & $1.0 \mathrm{E}+5$ \\
\hline Kramer 1996 & diaminostilbene DAS 2 & diarylethene photohydration & 3.33 & $1.5 \mathrm{E}-5$ & $4.7 \mathrm{E}+4$ & $4.7 \mathrm{E}+4$ & 0.72 & $1.1 \mathrm{E}-5$ & $6.6 \mathrm{E}+4$ \\
\hline $\begin{array}{l}\text { DellaGreca } \\
2008^{81}\end{array}$ & trazodone & aromatic halide photohydrolysis & 1.00 & $1.4 \mathrm{E}-6$ & $5.0 \mathrm{E}+5$ & $5.0 \mathrm{E}+5$ & 0.27 & $3.7 \mathrm{E}-7$ & $1.9 \mathrm{E}+6$ \\
\hline Astarita $2007^{82}$ & rosuvastatin & $\begin{array}{l}\text { o-vinylbiphenyl photocyclization } \\
\text { to dihydrophenanthrene }\end{array}$ & 0.30 & $2.7 \mathrm{E}-5$ & $2.6 \mathrm{E}+4$ & $2.6 \mathrm{E}+4$ & 0.32 & $8.6 \mathrm{E}-6$ & $8.1 \mathrm{E}+4$ \\
\hline Cermola $2007^{83}$ & fluvastatin & $\begin{array}{l}\text { o-vinylbiphenyl photocyclization } \\
\text { to dihydrophenanthrene } \\
\end{array}$ & 1.00 & $3.2 \mathrm{E}-5$ & $2.2 \mathrm{E}+4$ & $2.2 \mathrm{E}+4$ & 0.32 & $1.0 \mathrm{E}-5$ & $6.8 \mathrm{E}+4$ \\
\hline Zepp $1998^{84}$ & valerophenone & $\begin{array}{l}\text { aromatic ketone norrish II } \\
\text { photocleavage (C1_C4) }\end{array}$ & 3.33 & $1.2 \mathrm{E}-4$ & $6.0 \mathrm{E}+3$ & $6.0 \mathrm{E}+3$ & 0.65 & $7.5 \mathrm{E}-5$ & $9.2 \mathrm{E}+3$ \\
\hline Zepp 1998 & valerophenone & $\begin{array}{l}\text { aromatic ketone norrish II } \\
\text { photocleavage (C1_C4) }\end{array}$ & 3.33 & $1.2 \mathrm{E}-4$ & $6.0 \mathrm{E}+3$ & $6.0 \mathrm{E}+3$ & 0.65 & $7.5 \mathrm{E}-5$ & $9.2 \mathrm{E}+3$ \\
\hline Zepp 1998 & valerophenone & $\begin{array}{l}\text { aromatic ketone norrish II } \\
\text { photocyclization (C1_C4) }\end{array}$ & 3.33 & $1.2 \mathrm{E}-4$ & $6.0 \mathrm{E}+3$ & $6.0 \mathrm{E}+3$ & 0.32 & $3.7 \mathrm{E}-5$ & $1.9 \mathrm{E}+4$ \\
\hline Ware $1980^{85}$ & $\begin{array}{l}\text { 2,2'-bis(4- } \\
\text { chlorophenyl)acetic } \\
\text { acid }\end{array}$ & $\begin{array}{l}\text { aromatic acetic acid } \\
\text { photodecarboxylation to carbonyl }\end{array}$ & 1.00 & $2.4 \mathrm{E}-7$ & $2.9 \mathrm{E}+6$ & $2.9 \mathrm{E}+6$ & 0.40 & $9.5 \mathrm{E}-8$ & $7.3 \mathrm{E}+6$ \\
\hline Huang $2000^{86}$ & thioanisole & aromatic thioether photooxidation & 5.10 & $2.3 \mathrm{E}-5$ & $3.0 \mathrm{E}+4$ & $3.0 \mathrm{E}+4$ & 0.06 & $1.4 \mathrm{E}-6$ & $4.9 \mathrm{E}+5$ \\
\hline
\end{tabular}




\begin{tabular}{|l|l|l|l|l|l|l|l|l|l|}
\hline reference & contaminant & reaction scheme & $\beta$ & $\begin{array}{l}k_{\mathrm{pa}} \\
\left(\mathrm{s}^{-1}\right)\end{array}$ & $\begin{array}{l}t_{1 / 2, \mathrm{pa}} \\
(\mathrm{s})\end{array}$ & $\begin{array}{l}t_{1 / 2, \mathrm{pa}, \text { avg }} \\
(\mathrm{s})\end{array}$ & $\begin{array}{l}\alpha \\
k_{\mathrm{pr}, \text { avg }} \\
\left(\mathrm{s}^{-1}\right)\end{array}$ & $\begin{array}{l}t_{1 / 2, \mathrm{pr}, \mathrm{avg}} \\
(\mathrm{s})\end{array}$ \\
\hline Brigante $2005^{87}$ & hydrochlorothiazide & aromatic halide photohydrolysis & 0.30 & $2.1 \mathrm{E}-5$ & $3.2 \mathrm{E}+4$ & $3.2 \mathrm{E}+4$ & 0.75 & $1.6 \mathrm{E}-5$ & $4.3 \mathrm{E}+4$ \\
\hline Sharma $2014^{88}$ & cyantraniliprole & $\begin{array}{l}\text { anthranilic diamide } \\
\text { dehalogenative photocyclization } \\
\text { to oxazine }\end{array}$ & 3.04 & $1.6 \mathrm{E}-5$ & $4.5 \mathrm{E}+4$ & $4.5 \mathrm{E}+4$ & 1.00 & $1.6 \mathrm{E}-5$ & $4.5 \mathrm{E}+4$ \\
\hline $\begin{array}{l}\text { DellaGreca } \\
2007^{89}\end{array}$ & amlodipine & $\begin{array}{l}\text { dihydropyridine photooxidation } \\
\text { to pyridine }\end{array}$ & 1.67 & $1.1 \mathrm{E}-5$ & $6.0 \mathrm{E}+4$ & $6.0 \mathrm{E}+4$ & 0.73 & $8.4 \mathrm{E}-6$ & $8.3 \mathrm{E}+4$ \\
\hline $\begin{array}{l}\text { DellaGreca } \\
2004^{90}\end{array}$ & carboxin & $\begin{array}{l}\text { dihydrooxathiine anilide } \\
\text { photooxidation to sulfoxide }\end{array}$ & 1.00 & $2.0 \mathrm{E}-6$ & $3.5 \mathrm{E}+5$ & $3.5 \mathrm{E}+5$ & 0.51 & $1.0 \mathrm{E}-6$ & $6.8 \mathrm{E}+5$ \\
\hline
\end{tabular}

3 Note: Data are for all studies with a valid $\alpha$ value. $k_{\mathrm{pa}}$ and $t_{1 / 2 \text {,pa }}$ are parent degradation kinetics parameters calculated from the original

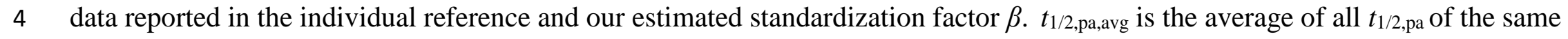
5 parent contaminants from different studies. Some referenced studies in the table only have information on the formation ratio $\alpha$ but the

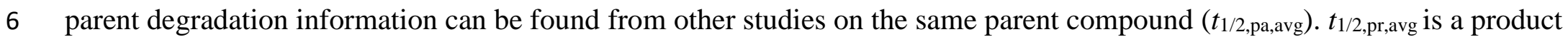

7 formation parameter calculated as the average of all $t_{1 / 2, \text { pa,avg }} / \alpha$ of the same parent contaminant and the same reaction scheme. $k_{\mathrm{pr}, \text { avg }}=$

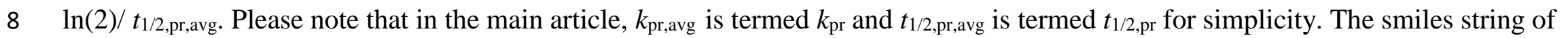
9 the contaminants can be found in the excel file associated with the supporting information. 
10 Table S2. Parent degradation and product formation rates from DB-EFSA-ENV.

\begin{tabular}{|c|c|c|c|c|c|c|c|c|c|c|c|c|}
\hline reference & contaminant & reaction scheme & $\beta_{\exp }$ & $\begin{array}{l}k_{\mathrm{pa}, \exp } \\
\left(\mathrm{s}^{-1}\right)\end{array}$ & $\begin{array}{l}t_{1 / 2, \mathrm{pa}, \exp } \\
(\mathrm{s})\end{array}$ & $\beta_{\text {mod }}$ & $\begin{array}{l}k_{\mathrm{pa}, \text { mod }} \\
\left(\mathrm{s}^{-1}\right)\end{array}$ & $\begin{array}{l}t_{1 / 2, \text { pa,mod }} \\
(\mathrm{s}) \\
\end{array}$ & $\begin{array}{l}t_{1 / 2, \text { pa,avg }} \\
(\mathrm{s})\end{array}$ & $\alpha$ & $\begin{array}{l}k_{\text {pravg }} \\
\left(\mathrm{s}^{-1}\right)\end{array}$ & $\begin{array}{l}t_{1 / 2, \mathrm{pr}, \mathrm{avg}} \\
\text { (s) }\end{array}$ \\
\hline $\begin{array}{l}\text { EFSA report on } \\
\text { trifluralin }\end{array}$ & trifluralin & $\begin{array}{l}\text { dinitroaniline } \\
\text { photochemical N- } \\
\text { dealkylation }\end{array}$ & 1.17 & $2.3 \mathrm{E}-5$ & $3.0 \mathrm{E}+4$ & & & & $3.0 \mathrm{E}+4$ & 0.08 & $1.9 \mathrm{E}-6$ & $3.7 \mathrm{E}+5$ \\
\hline $\begin{array}{l}\text { EFSA report on } \\
\text { bifenox }\end{array}$ & bifenox & $\begin{array}{l}\text { diphenyl ether } \\
\text { photohydrolysis }\end{array}$ & 2.15 & $3.7 \mathrm{E}-6$ & $1.9 \mathrm{E}+5$ & & & & $1.9 \mathrm{E}+5$ & 0.91 & $3.3 \mathrm{E}-6$ & $2.1 \mathrm{E}+5$ \\
\hline $\begin{array}{l}\text { EFSA report on } \\
\text { bitertanol }\end{array}$ & bitertanol & $\begin{array}{l}\text { aromatic ether } \\
\text { photohydrolysis }\end{array}$ & 6.01 & $4.9 \mathrm{E}-7$ & $1.4 \mathrm{E}+6$ & 1.00 & $4.5 \mathrm{E}-7$ & $1.6 \mathrm{E}+6$ & $1.6 \mathrm{E}+6$ & 0.43 & $1.8 \mathrm{E}-7$ & $3.8 \mathrm{E}+6$ \\
\hline $\begin{array}{l}\text { EFSA report on } \\
\text { dichlorprop-p }\end{array}$ & dichlorprop & $\begin{array}{l}\text { aromatic ether } \\
\text { photohydrolysis }\end{array}$ & 3.33 & $6.0 \mathrm{E}-7$ & $1.2 \mathrm{E}+6$ & & & & $1.2 \mathrm{E}+6$ & 0.003 & $1.9 \mathrm{E}-9$ & $3.7 \mathrm{E}+8$ \\
\hline $\begin{array}{l}\text { EFSA report on 1- } \\
\text { naphthaleneacetic acid }\end{array}$ & $\begin{array}{l}\text { 1-naphthaleneacetic } \\
\text { acid }\end{array}$ & $\begin{array}{l}\text { aromatic acetic acid } \\
\text { photodecarboxylation } \\
\text { to carbonyl }\end{array}$ & & & & 1.00 & $1.4 \mathrm{E}-6$ & $5.0 \mathrm{E}+5$ & $3.6 \mathrm{E}+5$ & 0.20 & $4.4 \mathrm{E}-7$ & $1.6 \mathrm{E}+6$ \\
\hline $\begin{array}{l}\text { EFSA report on 1- } \\
\text { naphthaleneacetic acid }\end{array}$ & $\begin{array}{l}\text { 1-naphthaleneacetic } \\
\text { acid }\end{array}$ & $\begin{array}{l}\text { aromatic acetic acid } \\
\text { photodecarboxylation } \\
\text { to carbonyl }\end{array}$ & 3.02 & $2.2 \mathrm{E}-6$ & $3.2 \mathrm{E}+5$ & 1.00 & $2.8 \mathrm{E}-6$ & $2.5 \mathrm{E}+5$ & $3.6 \mathrm{E}+5$ & 0.25 & $4.4 \mathrm{E}-7$ & $1.6 \mathrm{E}+6$ \\
\hline $\begin{array}{l}\text { EFSA report on 1- } \\
\text { naphthaleneacetic acid }\end{array}$ & $\begin{array}{l}\text { 1-naphthaleneacetic } \\
\text { acid }\end{array}$ & $\begin{array}{l}\text { aromatic acetic acid } \\
\text { photodecarboxylation } \\
\text { to alcohol }\end{array}$ & 3.02 & $2.2 \mathrm{E}-6$ & $3.2 \mathrm{E}+5$ & 1.00 & $2.8 \mathrm{E}-6$ & $2.5 \mathrm{E}+5$ & $3.6 \mathrm{E}+5$ & 0.02 & $4.3 \mathrm{E}-8$ & $1.6 \mathrm{E}+7$ \\
\hline $\begin{array}{l}\text { EFSA report on 1- } \\
\text { naphthaleneacetic acid }\end{array}$ & $\begin{array}{l}\text { 1-naphthaleneacetic } \\
\text { acid }\end{array}$ & $\begin{array}{l}\text { aromatic acetic acid } \\
\text { photodecarboxylation }\end{array}$ & 3.02 & $2.2 \mathrm{E}-6$ & $3.2 \mathrm{E}+5$ & 1.00 & $2.8 \mathrm{E}-6$ & $2.5 \mathrm{E}+5$ & $3.6 \mathrm{E}+5$ & 0.06 & $1.2 \mathrm{E}-7$ & $5.6 \mathrm{E}+6$ \\
\hline $\begin{array}{l}\text { EFSA report on } \\
\text { acrinathrin }\end{array}$ & acrinathrin & $\begin{array}{l}\text { pyrethroid carboxylic } \\
\text { acid ester } \\
\text { photohydrolysis }\end{array}$ & 1.00 & $2.1 \mathrm{E}-6$ & $3.2 \mathrm{E}+5$ & & & & $6.1 \mathrm{E}+5$ & 0.22 & $2.5 \mathrm{E}-7$ & $2.8 \mathrm{E}+6$ \\
\hline $\begin{array}{l}\text { EFSA report on } \\
\text { amisulbrom }\end{array}$ & amisulbrom & $\begin{array}{l}\text { sulfonamide } \\
\text { photohydrolysis }\end{array}$ & 2.83 & $1.1 \mathrm{E}-5$ & $6.2 \mathrm{E}+4$ & 3.33 & $1.0 \mathrm{E}-5$ & $6.8 \mathrm{E}+4$ & $6.5 \mathrm{E}+4$ & 0.08 & $3.3 \mathrm{E}-6$ & $2.1 \mathrm{E}+5$ \\
\hline $\begin{array}{l}\text { EFSA report on } \\
\text { amisulbrom }\end{array}$ & amisulbrom & $\begin{array}{l}\text { sulfonamide } \\
\text { photohydrolysis }\end{array}$ & 2.83 & $1.1 \mathrm{E}-5$ & $6.2 \mathrm{E}+4$ & 3.33 & $1.0 \mathrm{E}-5$ & $6.8 \mathrm{E}+4$ & $6.5 \mathrm{E}+4$ & 0.54 & $3.3 \mathrm{E}-6$ & $2.1 \mathrm{E}+5$ \\
\hline $\begin{array}{l}\text { EFSA report on } \\
\text { benzovindiflupyr }\end{array}$ & benzovindiflupyr & $\begin{array}{l}\mathrm{N} \text {-aryl amide } \\
\text { photohydrolysis }\end{array}$ & 2.85 & $6.4 \mathrm{E}-8$ & $1.1 \mathrm{E}+7$ & 1.00 & $8.4 \mathrm{E}-8$ & $8.2 \mathrm{E}+6$ & $9.6 \mathrm{E}+6$ & 0.07 & $4.8 \mathrm{E}-9$ & $1.4 \mathrm{E}+8$ \\
\hline $\begin{array}{l}\text { EFSA report on } \\
\text { bifenthrin }\end{array}$ & bifenthrin & $\begin{array}{l}\text { pyrethroid carboxylic } \\
\text { acid ester } \\
\text { photohydrolysis }\end{array}$ & & & & & & & $1.5 \mathrm{E}+6$ & 0.55 & $1.3 \mathrm{E}-7$ & $5.5 \mathrm{E}+6$ \\
\hline
\end{tabular}




\begin{tabular}{|c|c|c|c|c|c|c|c|c|c|c|c|c|}
\hline reference & contaminant & reaction scheme & $\beta_{\exp }$ & $\begin{array}{l}k_{\mathrm{pa}, \mathrm{exp}} \\
\left(\mathrm{s}^{-1}\right)\end{array}$ & $\begin{array}{l}t_{1 / 2, \mathrm{pa}, \exp } \\
(\mathrm{s})\end{array}$ & $\beta_{\text {mod }}$ & $\begin{array}{l}k_{\mathrm{pa}, \text { mod }} \\
\left(\mathrm{s}^{-1}\right)\end{array}$ & $\begin{array}{l}t_{1 / 2, \mathrm{pa} \text { mod }} \\
(\mathrm{s})\end{array}$ & $\begin{array}{l}t_{1 / 2, \mathrm{pa} \text {,avg }} \\
(\mathrm{s})\end{array}$ & $\alpha$ & $\begin{array}{l}k_{\text {pr,avg }} \\
\left(\mathrm{s}^{-1}\right)\end{array}$ & $\begin{array}{l}t_{1 / 2, \mathrm{pr} \text {,avg }} \\
(\mathrm{s})\end{array}$ \\
\hline $\begin{array}{l}\text { EFSA report on } \\
\text { bifenthrin }\end{array}$ & bifenthrin & $\begin{array}{l}\text { pyrethroid carboxylic } \\
\text { acid ester } \\
\text { photohydrolysis }\end{array}$ & 3.11 & $4.5 \mathrm{E}-7$ & $1.5 \mathrm{E}+6$ & 1.00 & $4.8 \mathrm{E}-7$ & $1.5 \mathrm{E}+6$ & $1.5 \mathrm{E}+6$ & 0.17 & $1.3 \mathrm{E}-7$ & $5.5 \mathrm{E}+6$ \\
\hline $\begin{array}{l}\text { EFSA report on } \\
\text { bifenthrin }\end{array}$ & bifenthrin & $\begin{array}{l}\text { pyrethroid carboxylic } \\
\text { acid ester } \\
\text { photohydrolysis }\end{array}$ & 3.11 & $4.5 \mathrm{E}-7$ & $1.5 \mathrm{E}+6$ & 1.00 & $4.8 \mathrm{E}-7$ & $1.5 \mathrm{E}+6$ & $1.5 \mathrm{E}+6$ & 0.10 & $1.3 \mathrm{E}-7$ & $5.5 \mathrm{E}+6$ \\
\hline $\begin{array}{l}\text { EFSA report on } \\
\text { bupirimate }\end{array}$ & bupirimate & $\begin{array}{l}\text { aromatic sulfonate } \\
\text { photohydrolysis }\end{array}$ & 1.00 & $4.0 \mathrm{E}-4$ & $1.7 \mathrm{E}+3$ & & & & $1.7 \mathrm{E}+3$ & 0.33 & $1.3 \mathrm{E}-4$ & $5.2 \mathrm{E}+3$ \\
\hline $\begin{array}{l}\text { EFSA report on } \\
\text { carboxin }\end{array}$ & carboxin & $\begin{array}{l}\text { dihydrooxathiine } \\
\text { anilide } \\
\text { photooxidation to } \\
\text { sulfoxide }\end{array}$ & 4.00 & $3.1 \mathrm{E}-5$ & $2.2 \mathrm{E}+4$ & 1.00 & $3.3 \mathrm{E}-4$ & $2.1 \mathrm{E}+3$ & $1.2 \mathrm{E}+4$ & 0.28 & $1.6 \mathrm{E}-5$ & $4.4 \mathrm{E}+4$ \\
\hline $\begin{array}{l}\text { EFSA report on } \\
\text { chlorantraniliprole }\end{array}$ & chlorantraniliprole & $\begin{array}{l}\text { anthranilic diamide } \\
\text { dehalogenative } \\
\text { photocyclization to } \\
\text { oxazine }\end{array}$ & 3.04 & $7.1 \mathrm{E}-6$ & $9.7 \mathrm{E}+4$ & 1.00 & $1.3 \mathrm{E}-5$ & $5.2 \mathrm{E}+4$ & $4.6 \mathrm{E}+5$ & 0.36 & $5.4 \mathrm{E}-7$ & $1.3 \mathrm{E}+6$ \\
\hline $\begin{array}{l}\text { EFSA report on } \\
\text { cyantraniliprole }\end{array}$ & cyantraniliprole & $\begin{array}{l}\text { anthranilic diamide } \\
\text { dehalogenative } \\
\text { photocyclization to } \\
\text { oxazine }\end{array}$ & 3.04 & $1.5 \mathrm{E}-5$ & $4.5 \mathrm{E}+4$ & & & & $4.5 \mathrm{E}+4$ & 0.91 & $1.4 \mathrm{E}-5$ & $4.9 \mathrm{E}+4$ \\
\hline $\begin{array}{l}\text { EFSA report on } \\
\text { cyantraniliprole }\end{array}$ & cyantraniliprole & $\begin{array}{l}\text { aromatic halide } \\
\text { photohydrolysis }\end{array}$ & 3.04 & $1.5 \mathrm{E}-5$ & $4.5 \mathrm{E}+4$ & & & & $4.5 \mathrm{E}+4$ & 0.02 & $3.5 \mathrm{E}-7$ & $2.0 \mathrm{E}+6$ \\
\hline $\begin{array}{l}\text { EFSA report on } \\
\text { sedaxane }\end{array}$ & sedaxane & $\begin{array}{l}\mathrm{N} \text {-aryl amide } \\
\text { photohydrolysis }\end{array}$ & 1.50 & $1.1 \mathrm{E}-7$ & $6.0 \mathrm{E}+6$ & 1.00 & $1.5 \mathrm{E}-7$ & $4.5 \mathrm{E}+6$ & $5.3 \mathrm{E}+6$ & 0.33 & $4.4 \mathrm{E}-8$ & $1.6 \mathrm{E}+7$ \\
\hline $\begin{array}{l}\text { EFSA report on } \\
\text { diethofencarb }\end{array}$ & diethofencarb & $\begin{array}{l}\text { aromatic carbamate } \\
\text { photohydrolysis }\end{array}$ & 1.00 & $4.6 \mathrm{E}-7$ & $1.5 \mathrm{E}+6$ & 1.00 & $2.0 \mathrm{E}-7$ & $3.5 \mathrm{E}+6$ & $3.1 \mathrm{E}+6$ & 0.14 & $3.1 \mathrm{E}-8$ & $2.3 \mathrm{E}+7$ \\
\hline $\begin{array}{l}\text { EFSA report on } \\
\text { diflufenican }\end{array}$ & diflufenican & $\begin{array}{l}\mathrm{N} \text {-aryl amide } \\
\text { photohydrolysis }\end{array}$ & 1.95 & $3.1 \mathrm{E}-8$ & $2.2 \mathrm{E}+7$ & 1.00 & 7.7E-8 & $9.0 \mathrm{E}+6$ & $1.6 \mathrm{E}+7$ & 0.08 & $3.6 \mathrm{E}-9$ & $1.9 \mathrm{E}+8$ \\
\hline $\begin{array}{l}\text { EFSA report on } \\
\text { dimethenamid }\end{array}$ & dimethenamid & $\begin{array}{l}\text { acetanilide O-dealkyl } \\
\text { dehalogenative } \\
\text { photocyclization to } \\
\text { morpholinone }\end{array}$ & 7.33 & $8.0 \mathrm{E}-8$ & $8.7 \mathrm{E}+6$ & & & & $4.6 \mathrm{E}+6$ & 0.003 & $\begin{array}{l}4.4 \mathrm{E}- \\
10\end{array}$ & $1.6 \mathrm{E}+9$ \\
\hline $\begin{array}{l}\text { EFSA report on } \\
\text { sulfosulfuron }\end{array}$ & sulfosulfuron & $\begin{array}{l}\text { sulfonylurea } \\
\text { photohydrolysis }\end{array}$ & 3.25 & $1.6 \mathrm{E}-6$ & $4.3 \mathrm{E}+5$ & 3.33 & $8.6 \mathrm{E}-7$ & $8.1 \mathrm{E}+5$ & $3.3 \mathrm{E}+5$ & 0.18 & $6.4 \mathrm{E}-7$ & $1.1 \mathrm{E}+6$ \\
\hline
\end{tabular}




\begin{tabular}{|c|c|c|c|c|c|c|c|c|c|c|c|c|}
\hline reference & contaminant & reaction scheme & $\beta_{\exp }$ & $\begin{array}{l}k_{\mathrm{pa,exp}} \\
\left(\mathrm{s}^{-1}\right)\end{array}$ & $\begin{array}{l}t_{1 / 2, \mathrm{pa}, \exp } \\
(\mathrm{s})\end{array}$ & $\beta_{\text {mod }}$ & $\begin{array}{l}k_{\mathrm{pa}, \bmod } \\
\left(\mathrm{s}^{-1}\right)\end{array}$ & $\begin{array}{l}t_{1 / 2, \mathrm{pa}, \bmod } \\
(\mathrm{s})\end{array}$ & $\begin{array}{l}t_{1 / 2, \mathrm{pa}, \mathrm{avg}} \\
(\mathrm{s})\end{array}$ & $\alpha$ & $\begin{array}{l}k_{\mathrm{pr}, \text { avg }} \\
\left(\mathrm{s}^{-1}\right)\end{array}$ & $\begin{array}{l}t_{1 / 2, \mathrm{pr}, \mathrm{avg}} \\
(\mathrm{s})\end{array}$ \\
\hline $\begin{array}{l}\text { EFSA report on } \\
\text { sulfosulfuron }\end{array}$ & sulfosulfuron & $\begin{array}{l}\text { sulfonylurea S-C } \\
\text { photohydrolysis }\end{array}$ & 3.25 & $1.6 \mathrm{E}-6$ & $4.3 \mathrm{E}+5$ & 3.33 & $8.6 \mathrm{E}-7$ & $8.1 \mathrm{E}+5$ & $3.3 \mathrm{E}+5$ & 0.23 & $5.2 \mathrm{E}-7$ & $1.3 \mathrm{E}+6$ \\
\hline $\begin{array}{l}\text { EFSA report on } \\
\text { sulfosulfuron }\end{array}$ & sulfosulfuron & $\begin{array}{l}\text { sulfonylurea S-N } \\
\text { photohydrolysis }\end{array}$ & 3.25 & $1.6 \mathrm{E}-6$ & $4.3 \mathrm{E}+5$ & 3.33 & $8.6 \mathrm{E}-7$ & $8.1 \mathrm{E}+5$ & $3.3 \mathrm{E}+5$ & 0.21 & $2.6 \mathrm{E}-7$ & $2.7 \mathrm{E}+6$ \\
\hline $\begin{array}{l}\text { EFSA report on } \\
\text { sulfosulfuron }\end{array}$ & sulfosulfuron & $\begin{array}{l}\text { sulfonylurea S-N } \\
\text { photohydrolysis }\end{array}$ & 3.25 & $1.6 \mathrm{E}-6$ & $4.3 \mathrm{E}+5$ & 3.33 & $8.6 \mathrm{E}-7$ & $8.1 \mathrm{E}+5$ & $3.3 \mathrm{E}+5$ & 0.12 & $2.6 \mathrm{E}-7$ & $2.7 \mathrm{E}+6$ \\
\hline $\begin{array}{l}\text { EFSA report on } \\
\text { sulfosulfuron }\end{array}$ & sulfosulfuron & $\begin{array}{l}\text { sulfonylurea S-C } \\
\text { photohydrolysis }\end{array}$ & 3.25 & $1.6 \mathrm{E}-6$ & $4.3 \mathrm{E}+5$ & 3.33 & $8.6 \mathrm{E}-7$ & $8.1 \mathrm{E}+5$ & $3.3 \mathrm{E}+5$ & 0.39 & $5.2 \mathrm{E}-7$ & $1.3 \mathrm{E}+6$ \\
\hline $\begin{array}{l}\text { EFSA report on } \\
\text { sulfosulfuron }\end{array}$ & sulfosulfuron & $\begin{array}{l}\text { sulfonylurea } \\
\text { photohydrolysis }\end{array}$ & 1.54 & $3.7 \mathrm{E}-6$ & $1.9 \mathrm{E}+5$ & & & & $3.3 \mathrm{E}+5$ & 0.44 & $6.4 \mathrm{E}-7$ & $1.1 \mathrm{E}+6$ \\
\hline $\begin{array}{l}\text { EFSA report on } \\
\text { sulfosulfuron }\end{array}$ & sulfosulfuron & $\begin{array}{l}\text { sulfonylurea S-C } \\
\text { photohydrolysis }\end{array}$ & 1.54 & $3.7 \mathrm{E}-6$ & $1.9 \mathrm{E}+5$ & & & & $3.3 \mathrm{E}+5$ & 0.18 & $5.2 \mathrm{E}-7$ & $1.3 \mathrm{E}+6$ \\
\hline $\begin{array}{l}\text { EFSA report on } \\
\text { sulfosulfuron }\end{array}$ & sulfosulfuron & $\begin{array}{l}\text { sulfonylurea S-N } \\
\text { photohydrolysis }\end{array}$ & 1.54 & $3.7 \mathrm{E}-6$ & $1.9 \mathrm{E}+5$ & & & & $3.3 \mathrm{E}+5$ & 0.08 & $2.6 \mathrm{E}-7$ & $2.7 \mathrm{E}+6$ \\
\hline $\begin{array}{l}\text { EFSA report on } \\
\text { sulfosulfuron }\end{array}$ & sulfosulfuron & $\begin{array}{l}\text { sulfonylurea S-C } \\
\text { photohydrolysis }\end{array}$ & 1.54 & $3.7 \mathrm{E}-6$ & $1.9 \mathrm{E}+5$ & & & & $3.3 \mathrm{E}+5$ & 0.20 & $5.2 \mathrm{E}-7$ & $1.3 \mathrm{E}+6$ \\
\hline $\begin{array}{l}\text { EFSA report on } \\
\text { dimoxystrobin }\end{array}$ & dimoxystrobin & $\begin{array}{l}\text { aromatic ether } \\
\text { photohydrolysis }\end{array}$ & 4.92 & $2.5 \mathrm{E}-8$ & $2.8 \mathrm{E}+7$ & & & & $2.8 \mathrm{E}+7$ & 0.21 & $5.2 \mathrm{E}-9$ & $1.3 \mathrm{E}+8$ \\
\hline $\begin{array}{l}\text { EFSA report on } \\
\text { diphenylamine }\end{array}$ & diphenylamine & $\begin{array}{l}\text { diphenylamine } \\
\text { photocyclization to } \\
\text { carbazole }\end{array}$ & & & & & & & $4.4 \mathrm{E}+3$ & 0.69 & $1.1 \mathrm{E}-4$ & $6.4 \mathrm{E}+3$ \\
\hline $\begin{array}{l}\text { EFSA report on } \\
\text { fenvalerate/esfenvaler } \\
\text { ate }\end{array}$ & fenvalerate & $\begin{array}{l}\text { pyrethroid carboxylic } \\
\text { acid ester } \\
\text { photohydrolysis }\end{array}$ & 1.00 & $8.0 \mathrm{E}-7$ & $8.6 \mathrm{E}+5$ & & & & $4.3 \mathrm{E}+5$ & 0.35 & $3.4 \mathrm{E}-7$ & $2.1 \mathrm{E}+6$ \\
\hline $\begin{array}{l}\text { EFSA report on } \\
\text { fenvalerate/esfenvaler } \\
\text { ate }\end{array}$ & fenvalerate & $\begin{array}{l}\text { pyrethroid carboxylic } \\
\text { acid ester } \\
\text { photohydrolysis }\end{array}$ & 1.39 & $2.9 \mathrm{E}-6$ & $2.4 \mathrm{E}+5$ & 1.00 & $4.0 \mathrm{E}-6$ & $1.7 \mathrm{E}+5$ & $4.3 \mathrm{E}+5$ & 0.07 & $3.4 \mathrm{E}-7$ & $2.1 \mathrm{E}+6$ \\
\hline $\begin{array}{l}\text { EFSA report on } \\
\text { fenvalerate/esfenvaler } \\
\text { ate }\end{array}$ & fenvalerate & $\begin{array}{l}\text { diphenyl ether } \\
\text { photohydrolysis }\end{array}$ & 1.39 & $2.9 \mathrm{E}-6$ & $2.4 \mathrm{E}+5$ & 1.00 & $4.0 \mathrm{E}-6$ & $1.7 \mathrm{E}+5$ & $4.3 \mathrm{E}+5$ & 0.03 & $4.1 \mathrm{E}-8$ & $1.7 \mathrm{E}+7$ \\
\hline
\end{tabular}




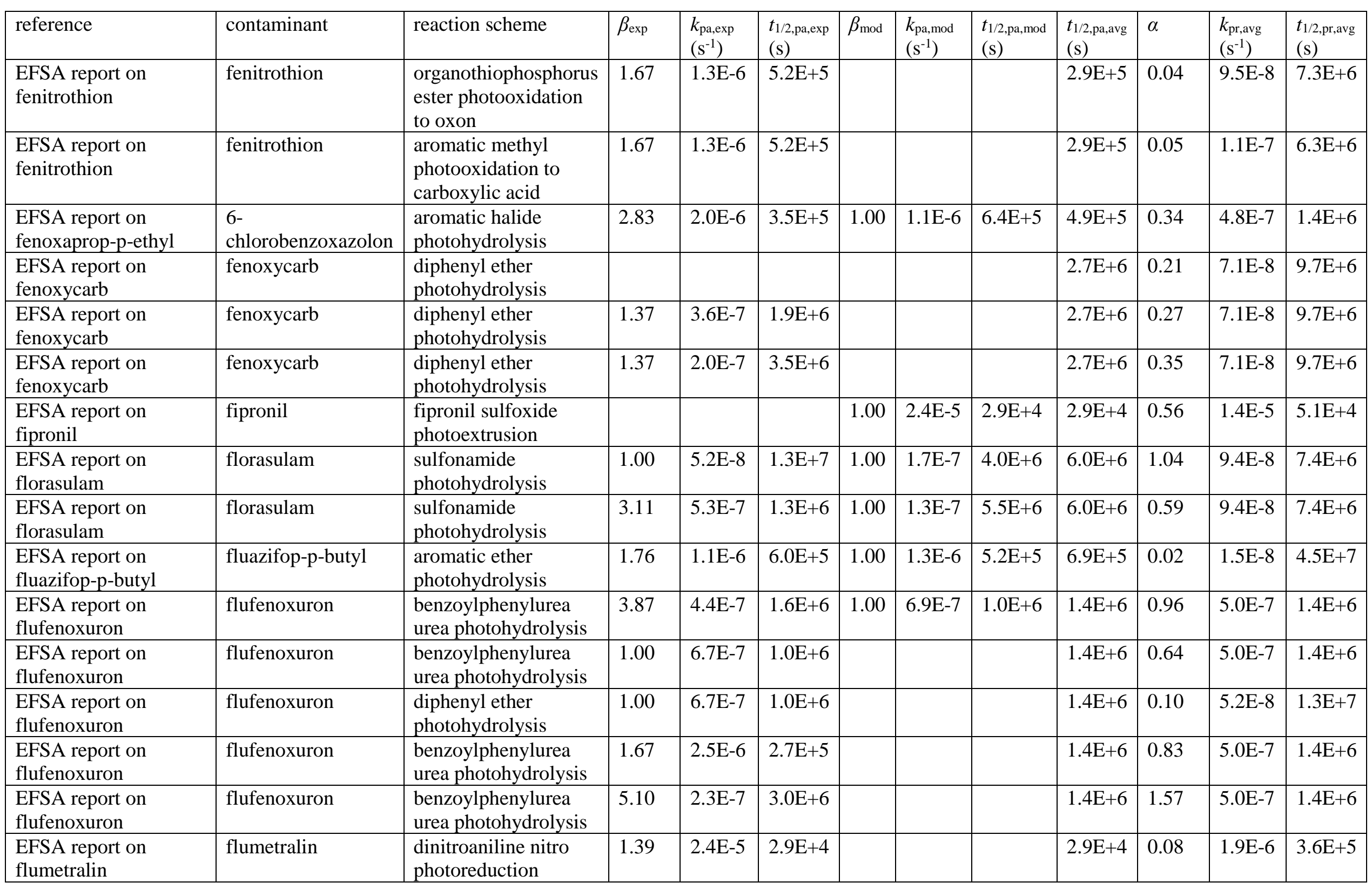




\begin{tabular}{|c|c|c|c|c|c|c|c|c|c|c|c|c|}
\hline reference & contaminant & reaction scheme & $\beta_{\exp }$ & $\begin{array}{l}k_{\mathrm{pa}, \exp } \\
\left(\mathrm{s}^{-1}\right)\end{array}$ & $\begin{array}{l}t_{1 / 2, \mathrm{pa} \text {,exp }} \\
\text { (s) }\end{array}$ & $\beta_{\text {mod }}$ & $\begin{array}{l}k_{\mathrm{pa}, \text { mod }} \\
\left(\mathrm{s}^{-1}\right)\end{array}$ & $\begin{array}{l}t_{1 / 2, \mathrm{pa} \text { mod }} \\
(\mathrm{s})\end{array}$ & $\begin{array}{l}t_{1 / 2, \text { pa,avg }} \\
(\mathrm{s})\end{array}$ & $\alpha$ & $\begin{array}{l}k_{\mathrm{pr}, \mathrm{avg}} \\
\left(\mathrm{s}^{-1}\right)\end{array}$ & $\begin{array}{l}t_{1 / 2, \mathrm{pr}, \mathrm{avg}} \\
\text { (s) }\end{array}$ \\
\hline $\begin{array}{l}\text { EFSA report on } \\
\text { gamma-cyhalothrin }\end{array}$ & cyhalothrin & $\begin{array}{l}\text { pyrethroid carboxylic } \\
\text { acid ester } \\
\text { photohydrolysis }\end{array}$ & 1.50 & 7.8E-7 & $8.9 \mathrm{E}+5$ & 1.00 & 7.6E-7 & $9.2 \mathrm{E}+5$ & $8.3 \mathrm{E}+5$ & 0.59 & $3.1 \mathrm{E}-7$ & $2.3 \mathrm{E}+6$ \\
\hline $\begin{array}{l}\text { EFSA report on } \\
\text { halauxifen-metyhl }\end{array}$ & halauxifen-metyhl & $\begin{array}{l}\text { aromatic halide } \\
\text { photohydrolysis }\end{array}$ & 2.02 & $1.4 \mathrm{E}-3$ & $5.0 \mathrm{E}+2$ & 1.00 & $1.5 \mathrm{E}-3$ & $4.6 \mathrm{E}+2$ & $4.8 \mathrm{E}+2$ & 0.05 & $7.5 \mathrm{E}-5$ & $9.3 \mathrm{E}+3$ \\
\hline $\begin{array}{l}\text { EFSA report on } \\
\text { haloxyfop-P }\end{array}$ & haloxyfop-P & $\begin{array}{l}\text { diphenyl ether } \\
\text { photohydrolysis }\end{array}$ & & & & 1.00 & $4.0 \mathrm{E}-7$ & $1.7 \mathrm{E}+6$ & $1.7 \mathrm{E}+6$ & 0.19 & 7.7E-8 & $9.0 \mathrm{E}+6$ \\
\hline $\begin{array}{l}\text { EFSA report on } \\
\text { haloxyfop-P }\end{array}$ & haloxyfop-P-acid & $\begin{array}{l}\text { diphenyl ether } \\
\text { photohydrolysis }\end{array}$ & & & & 1.00 & $6.7 \mathrm{E}-7$ & $1.0 \mathrm{E}+6$ & $1.0 \mathrm{E}+6$ & 0.13 & $8.6 \mathrm{E}-8$ & $8.1 \mathrm{E}+6$ \\
\hline $\begin{array}{l}\text { EFSA report on } \\
\text { haloxyfop-P }\end{array}$ & haloxyfop-P-acid & $\begin{array}{l}\text { aromatic ether } \\
\text { photohydrolysis }\end{array}$ & & & & 1.00 & $6.7 \mathrm{E}-7$ & $1.0 \mathrm{E}+6$ & $1.0 \mathrm{E}+6$ & 0.23 & $1.5 \mathrm{E}-7$ & $4.6 \mathrm{E}+6$ \\
\hline $\begin{array}{l}\text { EFSA report on } \\
\text { haloxyfop-P }\end{array}$ & haloxyfop-P-acid & $\begin{array}{l}\text { phenoxyacetic acid } \\
\text { photodecarboxylation } \\
\text { to carbonyl }\end{array}$ & & & & 1.00 & $6.7 \mathrm{E}-7$ & $1.0 \mathrm{E}+6$ & $1.0 \mathrm{E}+6$ & 0.24 & $1.6 \mathrm{E}-7$ & $4.2 \mathrm{E}+6$ \\
\hline $\begin{array}{l}\text { EFSA report on } \\
\text { imazaquin }\end{array}$ & imazaquin & $\begin{array}{l}\text { imidazolinone ring } \\
\text { photocleavage to } \\
\text { amide }\end{array}$ & 7.62 & $1.2 \mathrm{E}-6$ & $5.8 \mathrm{E}+5$ & & & & $4.7 \mathrm{E}+5$ & 0.11 & $1.6 \mathrm{E}-7$ & $4.3 \mathrm{E}+6$ \\
\hline $\begin{array}{l}\text { EFSA report on } \\
\text { imazaquin }\end{array}$ & imazaquin & $\begin{array}{l}\text { imidazolinone ring } \\
\text { photocleavage to } \\
\text { carboxylic acid }\end{array}$ & 7.62 & $1.2 \mathrm{E}-6$ & $5.8 \mathrm{E}+5$ & & & & $4.7 \mathrm{E}+5$ & 0.17 & $2.5 \mathrm{E}-7$ & $2.8 \mathrm{E}+6$ \\
\hline $\begin{array}{l}\text { EFSA report on } \\
\text { imazaquin }\end{array}$ & imazaquin & $\begin{array}{l}\text { imidazolinone ring } \\
\text { photocleavage to } \\
\text { amide }\end{array}$ & 3.33 & $1.9 \mathrm{E}-6$ & $3.6 \mathrm{E}+5$ & & & & $4.7 \mathrm{E}+5$ & 0.11 & $1.6 \mathrm{E}-7$ & $4.3 \mathrm{E}+6$ \\
\hline $\begin{array}{l}\text { EFSA report on } \\
\text { imidacloprid }\end{array}$ & imidacloprid & $\begin{array}{l}\text { nitroguanidine imine } \\
\text { photohydrolysis }\end{array}$ & & & & 1.00 & $4.6 \mathrm{E}-5$ & $1.5 \mathrm{E}+4$ & $1.4 \mathrm{E}+4$ & 0.14 & $6.8 \mathrm{E}-6$ & $1.0 \mathrm{E}+5$ \\
\hline $\begin{array}{l}\text { EFSA report on } \\
\text { imidacloprid }\end{array}$ & imidacloprid & $\begin{array}{l}\text { nitroguanidine } \\
\text { photochemical N- } \\
\text { denitration }\end{array}$ & & & & 1.00 & $4.6 \mathrm{E}-5$ & $1.5 \mathrm{E}+4$ & $1.4 \mathrm{E}+4$ & 0.24 & $1.2 \mathrm{E}-5$ & $5.8 \mathrm{E}+4$ \\
\hline $\begin{array}{l}\text { EFSA report on } \\
\text { isopyrazam }\end{array}$ & isopyrazam & $\begin{array}{l}\mathrm{N} \text {-aryl amide } \\
\text { photohydrolysis }\end{array}$ & 1.40 & $1.1 \mathrm{E}-7$ & $6.6 \mathrm{E}+6$ & 1.00 & $1.3 \mathrm{E}-7$ & $5.4 \mathrm{E}+6$ & $6.0 \mathrm{E}+6$ & 0.35 & $4.0 \mathrm{E}-8$ & $1.7 \mathrm{E}+7$ \\
\hline $\begin{array}{l}\text { EFSA report on } \\
\text { lambda-cyhalothrin }\end{array}$ & cyhalothrin & $\begin{array}{l}\text { pyrethroid carboxylic } \\
\text { acid ester } \\
\text { photohydrolysis }\end{array}$ & 2.98 & $7.2 \mathrm{E}-7$ & $9.6 \mathrm{E}+5$ & 1.00 & $9.4 \mathrm{E}-7$ & $7.3 \mathrm{E}+5$ & $8.3 \mathrm{E}+5$ & 0.14 & $3.1 \mathrm{E}-7$ & $2.3 \mathrm{E}+6$ \\
\hline
\end{tabular}




\begin{tabular}{|c|c|c|c|c|c|c|c|c|c|c|c|c|}
\hline reference & contaminant & reaction scheme & $\beta_{\text {exp }}$ & $\begin{array}{l}k_{\text {pa,exp }} \\
\left(\mathrm{s}^{-1}\right)\end{array}$ & $\begin{array}{l}t_{1 / 2, \mathrm{pa}, \exp } \\
(\mathrm{s})\end{array}$ & $\beta_{\text {mod }}$ & $\begin{array}{l}k_{\mathrm{pa}, \text { mod }} \\
\left(\mathrm{s}^{-1}\right)\end{array}$ & $\begin{array}{l}t_{1 / 2, \mathrm{pa} \text {,mod }} \\
(\mathrm{s})\end{array}$ & $\begin{array}{l}t_{1 / 2, \mathrm{pa}, \mathrm{avg}} \\
(\mathrm{s})\end{array}$ & $\alpha$ & $\begin{array}{l}k_{\mathrm{pr}, \text { avg }} \\
\left(\mathrm{s}^{-1}\right)\end{array}$ & $\begin{array}{l}t_{1 / 2, \text { pr,avg }} \\
\text { (s) }\end{array}$ \\
\hline $\begin{array}{l}\text { EFSA report on } \\
\text { lufenuron }\end{array}$ & lufenuron & $\begin{array}{l}\text { benzoylphenylurea } \\
\text { urea photohydrolysis }\end{array}$ & 1.38 & $5.6 \mathrm{E}-7$ & $1.2 \mathrm{E}+6$ & 1.00 & $4.3 \mathrm{E}-7$ & $1.6 \mathrm{E}+6$ & $2.1 \mathrm{E}+6$ & 0.64 & $1.6 \mathrm{E}-7$ & $4.4 \mathrm{E}+6$ \\
\hline $\begin{array}{l}\text { EFSA report on } \\
\text { lufenuron }\end{array}$ & lufenuron & $\begin{array}{l}\text { benzoylphenylurea } \\
\text { urea photohydrolysis }\end{array}$ & 1.96 & $2.6 \mathrm{E}-7$ & $2.7 \mathrm{E}+6$ & 1.00 & $2.4 \mathrm{E}-7$ & $2.9 \mathrm{E}+6$ & $2.1 \mathrm{E}+6$ & 0.31 & $1.6 \mathrm{E}-7$ & $4.4 \mathrm{E}+6$ \\
\hline $\begin{array}{l}\text { EFSA report on } \\
\text { mandestrobin }\end{array}$ & mandestrobin & $\begin{array}{l}\text { benzyl phenyl ether } \\
\text { photorearrangement } \\
\text { (o) }\end{array}$ & 1.39 & $1.3 \mathrm{E}-6$ & $5.3 \mathrm{E}+5$ & 1.00 & $1.8 \mathrm{E}-6$ & $3.8 \mathrm{E}+5$ & $4.6 \mathrm{E}+5$ & 0.31 & $4.4 \mathrm{E}-7$ & $1.6 \mathrm{E}+6$ \\
\hline $\begin{array}{l}\text { EFSA report on } \\
\text { mandestrobin }\end{array}$ & mandestrobin & $\begin{array}{l}\text { aromatic ether } \\
\text { photohydrolysis }\end{array}$ & 1.39 & $1.3 \mathrm{E}-6$ & $5.3 \mathrm{E}+5$ & 1.00 & $1.8 \mathrm{E}-6$ & $3.8 \mathrm{E}+5$ & $4.6 \mathrm{E}+5$ & 0.04 & $5.7 \mathrm{E}-8$ & $1.2 \mathrm{E}+7$ \\
\hline $\begin{array}{l}\text { EFSA report on } \\
\text { mandestrobin }\end{array}$ & mandestrobin & $\begin{array}{l}\text { benzyl phenyl ether } \\
\text { photorearrangement } \\
\text { (p) }\end{array}$ & 1.39 & $1.3 \mathrm{E}-6$ & $5.3 \mathrm{E}+5$ & 1.00 & $1.8 \mathrm{E}-6$ & $3.8 \mathrm{E}+5$ & $4.6 \mathrm{E}+5$ & 0.15 & $2.1 \mathrm{E}-7$ & $3.3 \mathrm{E}+6$ \\
\hline $\begin{array}{l}\text { EFSA report on } \\
\text { mandestrobin }\end{array}$ & mandestrobin & $\begin{array}{l}\text { benzyl phenyl ether } \\
\text { photorearrangement } \\
\text { (o) }\end{array}$ & 1.39 & $1.3 \mathrm{E}-6$ & $5.5 \mathrm{E}+5$ & 1.00 & $1.7 \mathrm{E}-6$ & $4.0 \mathrm{E}+5$ & $4.6 \mathrm{E}+5$ & 0.28 & $4.4 \mathrm{E}-7$ & $1.6 \mathrm{E}+6$ \\
\hline $\begin{array}{l}\text { EFSA report on } \\
\text { mandestrobin }\end{array}$ & mandestrobin & $\begin{array}{l}\text { aromatic ether } \\
\text { photohydrolysis }\end{array}$ & 1.39 & $1.3 \mathrm{E}-6$ & $5.5 \mathrm{E}+5$ & 1.00 & $1.7 \mathrm{E}-6$ & $4.0 \mathrm{E}+5$ & $4.6 \mathrm{E}+5$ & 0.04 & $5.7 \mathrm{E}-8$ & $1.2 \mathrm{E}+7$ \\
\hline $\begin{array}{l}\text { EFSA report on } \\
\text { mandestrobin }\end{array}$ & mandestrobin & $\begin{array}{l}\text { benzyl phenyl ether } \\
\text { photorearrangement } \\
\text { (p) }\end{array}$ & 1.39 & $1.3 \mathrm{E}-6$ & $5.5 \mathrm{E}+5$ & 1.00 & $1.7 \mathrm{E}-6$ & $4.0 \mathrm{E}+5$ & $4.6 \mathrm{E}+5$ & 0.14 & $2.1 \mathrm{E}-7$ & $3.3 \mathrm{E}+6$ \\
\hline $\begin{array}{l}\text { EFSA report on } \\
\text { metamitron }\end{array}$ & metamitron & $\begin{array}{l}\text { 1_2_4-triazine-5-one } \\
\text { photochemical N- } \\
\text { deamination }\end{array}$ & 1.00 & $1.7 \mathrm{E}-3$ & $4.2 \mathrm{E}+2$ & 1.00 & $4.5 \mathrm{E}-4$ & $1.5 \mathrm{E}+3$ & $9.8 \mathrm{E}+2$ & 0.87 & $6.2 \mathrm{E}-4$ & $1.1 \mathrm{E}+3$ \\
\hline $\begin{array}{l}\text { EFSA report on } \\
\text { methiocarb }\end{array}$ & methiocarb & $\begin{array}{l}\text { aromatic thioether } \\
\text { photooxidation }\end{array}$ & 5.90 & $1.7 \mathrm{E}-7$ & $4.2 \mathrm{E}+6$ & 1.00 & $1.7 \mathrm{E}-7$ & $4.1 \mathrm{E}+6$ & $5.2 \mathrm{E}+6$ & 0.81 & $1.1 \mathrm{E}-7$ & $6.5 \mathrm{E}+6$ \\
\hline $\begin{array}{l}\text { EFSA report on } \\
\text { metobromuron }\end{array}$ & metobromuron & $\begin{array}{l}\text { aromatic } \\
\text { photohydrodehalogen } \\
\text { ation }\end{array}$ & 2.11 & $6.8 \mathrm{E}-7$ & $1.0 \mathrm{E}+6$ & 1.00 & $6.6 \mathrm{E}-7$ & $1.0 \mathrm{E}+6$ & $1.0 \mathrm{E}+6$ & 0.14 & $9.7 \mathrm{E}-8$ & $7.2 \mathrm{E}+6$ \\
\hline $\begin{array}{l}\text { EFSA report on } \\
\text { metobromuron }\end{array}$ & metobromuron & $\begin{array}{l}\text { aromatic halide } \\
\text { photohydrolysis }\end{array}$ & 2.11 & $6.8 \mathrm{E}-7$ & $1.0 \mathrm{E}+6$ & 1.00 & $6.6 \mathrm{E}-7$ & $1.0 \mathrm{E}+6$ & $1.0 \mathrm{E}+6$ & 0.15 & $1.0 \mathrm{E}-7$ & $6.9 \mathrm{E}+6$ \\
\hline $\begin{array}{l}\text { EFSA report on } \\
\text { metrafenone }\end{array}$ & metrafenone & $\begin{array}{l}\text { aromatic halide } \\
\text { photohydrolysis }\end{array}$ & 2.18 & $1.1 \mathrm{E}-6$ & $6.1 \mathrm{E}+5$ & 1.00 & $6.5 \mathrm{E}-7$ & $1.1 \mathrm{E}+6$ & $8.3 \mathrm{E}+5$ & 0.10 & $8.6 \mathrm{E}-8$ & $8.0 \mathrm{E}+6$ \\
\hline $\begin{array}{l}\text { EFSA report on } \\
\text { metrafenone }\end{array}$ & metrafenone & $\begin{array}{l}\text { aromatic ether } \\
\text { photohydrolysis }\end{array}$ & 2.18 & $1.1 \mathrm{E}-6$ & $6.1 \mathrm{E}+5$ & 1.00 & $6.5 \mathrm{E}-7$ & $1.1 \mathrm{E}+6$ & $8.3 \mathrm{E}+5$ & 0.01 & $8.0 \mathrm{E}-9$ & $8.7 \mathrm{E}+7$ \\
\hline
\end{tabular}




\begin{tabular}{|c|c|c|c|c|c|c|c|c|c|c|c|c|}
\hline reference & contaminant & reaction scheme & $\beta_{\exp }$ & $\begin{array}{l}k_{\mathrm{pa}, \mathrm{exp}} \\
\left(\mathrm{s}^{-1}\right)\end{array}$ & $\begin{array}{l}t_{1 / 2, \mathrm{pa}, \exp } \\
(\mathrm{s})\end{array}$ & $\beta_{\text {mod }}$ & $\begin{array}{l}k_{\mathrm{pa}, \text { mod }} \\
\left(\mathrm{s}^{-1}\right)\end{array}$ & $\begin{array}{l}t_{1 / 2, \mathrm{pa} \text { mod }} \\
(\mathrm{s})\end{array}$ & $\begin{array}{l}t_{1 / 2, \mathrm{pa} \text {,avg }} \\
(\mathrm{s})\end{array}$ & $\alpha$ & $\begin{array}{l}k_{\text {pr,avg }} \\
\left(\mathrm{s}^{-1}\right)\end{array}$ & $\begin{array}{l}t_{1 / 2, \mathrm{pr} \text {,avg }} \\
\text { (s) }\end{array}$ \\
\hline $\begin{array}{l}\text { EFSA report on } \\
\text { metrafenone }\end{array}$ & metrafenone & $\begin{array}{l}\text { aromatic ether } \\
\text { photohydrolysis }\end{array}$ & 2.18 & $1.1 \mathrm{E}-6$ & $6.1 \mathrm{E}+5$ & 1.00 & $6.5 \mathrm{E}-7$ & $1.1 \mathrm{E}+6$ & $8.3 \mathrm{E}+5$ & 0.01 & $8.0 \mathrm{E}-9$ & $8.7 \mathrm{E}+7$ \\
\hline $\begin{array}{l}\text { EFSA report on } \\
\text { metrafenone }\end{array}$ & metrafenone & $\begin{array}{l}\text { aromatic } \\
\text { photohydrodehalogen } \\
\text { ation }\end{array}$ & 2.18 & $1.1 \mathrm{E}-6$ & $6.1 \mathrm{E}+5$ & 1.00 & $6.5 \mathrm{E}-7$ & $1.1 \mathrm{E}+6$ & $8.3 \mathrm{E}+5$ & 0.01 & $8.4 \mathrm{E}-9$ & $8.2 \mathrm{E}+7$ \\
\hline $\begin{array}{l}\text { EFSA report on } \\
\text { metsulfuron-methyl }\end{array}$ & metsulfuron-methyl & $\begin{array}{l}\text { sulfonylurea S-C } \\
\text { photohydrolysis }\end{array}$ & & & & & & & $2.4 \mathrm{E}+7$ & 0.57 & $1.6 \mathrm{E}-8$ & $4.2 \mathrm{E}+7$ \\
\hline $\begin{array}{l}\text { EFSA report on } \\
\text { metsulfuron-methyl }\end{array}$ & metsulfuron-methyl & $\begin{array}{l}\text { aromatic ether } \\
\text { photohydrolysis }\end{array}$ & 4.00 & $2.3 \mathrm{E}-8$ & $3.1 \mathrm{E}+7$ & 1.00 & $3.1 \mathrm{E}-8$ & $2.2 \mathrm{E}+7$ & $2.4 \mathrm{E}+7$ & 0.43 & $1.2 \mathrm{E}-8$ & $5.6 \mathrm{E}+7$ \\
\hline $\begin{array}{l}\text { EFSA report on } \\
\text { napropamide }\end{array}$ & napropamide & $\begin{array}{l}\text { 1-naphthoxy } \\
\text { photorearrangement } \\
\text { (C4) }\end{array}$ & 4.00 & $5.1 \mathrm{E}-4$ & $1.4 \mathrm{E}+3$ & 1.00 & $1.7 \mathrm{E}-3$ & $4.1 \mathrm{E}+2$ & $9.9 \mathrm{E}+2$ & 0.26 & $1.1 \mathrm{E}-4$ & $6.1 \mathrm{E}+3$ \\
\hline $\begin{array}{l}\text { EFSA report on } \\
\text { napropamide }\end{array}$ & napropamide & $\begin{array}{l}\text { 1-naphthoxy } \\
\text { photorearrangement } \\
\text { (C2) }\end{array}$ & 4.00 & $5.1 \mathrm{E}-4$ & $1.4 \mathrm{E}+3$ & 1.00 & $1.7 \mathrm{E}-3$ & $4.1 \mathrm{E}+2$ & $9.9 \mathrm{E}+2$ & 0.57 & $2.6 \mathrm{E}-4$ & $2.7 \mathrm{E}+3$ \\
\hline $\begin{array}{l}\text { EFSA report on } \\
\text { napropamide }\end{array}$ & napropamide & $\begin{array}{l}\text { 1-naphthoxy } \\
\text { photorearrangement } \\
\text { (C4) }\end{array}$ & & & & & & & $9.9 \mathrm{E}+2$ & 0.06 & $1.1 \mathrm{E}-4$ & $6.1 \mathrm{E}+3$ \\
\hline $\begin{array}{l}\text { EFSA report on } \\
\text { napropamide }\end{array}$ & napropamide & $\begin{array}{l}\text { 1-naphthoxy } \\
\text { photorearrangement } \\
\text { (C2) }\end{array}$ & & & & & & & $9.9 \mathrm{E}+2$ & 0.17 & $2.6 \mathrm{E}-4$ & $2.7 \mathrm{E}+3$ \\
\hline $\begin{array}{l}\text { EFSA report on } \\
\text { napropamide }\end{array}$ & napropamide & $\begin{array}{l}\text { aromatic ether } \\
\text { photohydrolysis }\end{array}$ & & & & & & & $9.9 \mathrm{E}+2$ & 0.04 & $3.0 \mathrm{E}-5$ & $2.3 \mathrm{E}+4$ \\
\hline $\begin{array}{l}\text { EFSA report on } \\
\text { novaluron }\end{array}$ & novaluron & $\begin{array}{l}\text { benzoylphenylurea } \\
\text { urea photohydrolysis }\end{array}$ & 16.67 & & & 1.00 & $5.8 \mathrm{E}-8$ & $1.2 \mathrm{E}+7$ & $1.2 \mathrm{E}+7$ & 3.54 & $2.0 \mathrm{E}-7$ & $3.4 \mathrm{E}+6$ \\
\hline $\begin{array}{l}\text { EFSA report on } \\
\text { oxyfluorfen }\end{array}$ & oxyfluorfen & $\begin{array}{l}\text { aromatic nitro } \\
\text { photohydrolysis }\end{array}$ & 2.00 & $2.3 \mathrm{E}-5$ & $3.1 \mathrm{E}+4$ & 1.00 & $2.6 \mathrm{E}-5$ & $2.7 \mathrm{E}+4$ & $2.9 \mathrm{E}+4$ & 0.17 & $4.1 \mathrm{E}-6$ & $1.7 \mathrm{E}+5$ \\
\hline $\begin{array}{l}\text { EFSA report on } \\
\text { pirimicarb }\end{array}$ & pirimicarb & $\begin{array}{l}\text { s-triazine side chain } \\
\mathrm{N} \text {-alkyl } \\
\text { photooxidation to } \\
\text { carbonyl }\end{array}$ & 1.29 & $7.8 \mathrm{E}-5$ & $8.8 \mathrm{E}+3$ & & & & $1.3 \mathrm{E}+4$ & 0.20 & $1.1 \mathrm{E}-5$ & $6.4 \mathrm{E}+4$ \\
\hline $\begin{array}{l}\text { EFSA report on } \\
\text { pirimicarb }\end{array}$ & pirimicarb & $\begin{array}{l}\text { s-triazine side chain } \\
\text { photochemical N- } \\
\text { dealkylation }\end{array}$ & 1.29 & $7.8 \mathrm{E}-5$ & $8.8 \mathrm{E}+3$ & & & & $1.3 \mathrm{E}+4$ & 0.08 & $4.3 \mathrm{E}-6$ & $1.6 \mathrm{E}+5$ \\
\hline
\end{tabular}




\begin{tabular}{|c|c|c|c|c|c|c|c|c|c|c|c|c|}
\hline reference & contaminant & reaction scheme & $\beta_{\exp }$ & $\begin{array}{l}k_{\mathrm{pa}, \exp } \\
\left(\mathrm{s}^{-1}\right)\end{array}$ & $\begin{array}{l}t_{1 / 2, \mathrm{pa}, \exp } \\
(\mathrm{s})\end{array}$ & $\beta_{\text {mod }}$ & $\begin{array}{l}k_{\text {pa,mod }} \\
\left(\mathrm{s}^{-1}\right)\end{array}$ & $\begin{array}{l}t_{1 / 2, \mathrm{pa} \text { mod }} \\
(\mathrm{s})\end{array}$ & $\begin{array}{l}t_{1 / 2, \text { pa,avg }} \\
(\mathrm{s})\end{array}$ & $\alpha$ & $\begin{array}{l}k_{\mathrm{pr}, \text { avg }} \\
\left(\mathrm{s}^{-1}\right)\end{array}$ & $\begin{array}{l}t_{1 / 2, \mathrm{pr} \text {,avg }} \\
\text { (s) }\end{array}$ \\
\hline $\begin{array}{l}\text { EFSA report on } \\
\text { pirimicarb }\end{array}$ & pirimicarb & $\begin{array}{l}\text { aromatic carbamate } \\
\text { photohydrolysis }\end{array}$ & 1.29 & $7.8 \mathrm{E}-5$ & $8.8 \mathrm{E}+3$ & & & & $1.3 \mathrm{E}+4$ & 0.26 & $1.4 \mathrm{E}-5$ & $5.1 \mathrm{E}+4$ \\
\hline $\begin{array}{l}\text { EFSA report on } \\
\text { propargite }\end{array}$ & propargite & $\begin{array}{l}\text { aromatic ether } \\
\text { photohydrolysis }\end{array}$ & 2.40 & $6.1 \mathrm{E}-7$ & $1.1 \mathrm{E}+6$ & 1.00 & $6.1 \mathrm{E}-7$ & $1.1 \mathrm{E}+6$ & $1.1 \mathrm{E}+6$ & 0.14 & $9.7 \mathrm{E}-8$ & $7.1 \mathrm{E}+6$ \\
\hline $\begin{array}{l}\text { EFSA report on } \\
\text { propargite }\end{array}$ & propargite & $\begin{array}{l}\text { aromatic ether } \\
\text { photohydrolysis }\end{array}$ & & & & 1.00 & $7.7 \mathrm{E}-7$ & $9.0 \mathrm{E}+5$ & $1.1 \mathrm{E}+6$ & 0.15 & $9.7 \mathrm{E}-8$ & $7.1 \mathrm{E}+6$ \\
\hline $\begin{array}{l}\text { EFSA report on } \\
\text { proquinazid }\end{array}$ & proquinazid & $\begin{array}{l}\text { aromatic } \\
\text { photohydrodehalogen } \\
\text { ation }\end{array}$ & 2.00 & $1.3 \mathrm{E}-4$ & $5.2 \mathrm{E}+3$ & & & & $5.2 \mathrm{E}+3$ & 0.13 & $1.8 \mathrm{E}-5$ & $3.8 \mathrm{E}+4$ \\
\hline $\begin{array}{l}\text { EFSA report on } \\
\text { pyridaben }\end{array}$ & pyridaben & $\begin{array}{l}\text { aromatic thioether } \\
\text { photooxidation }\end{array}$ & 2.83 & $3.5 \mathrm{E}-4$ & $2.0 \mathrm{E}+3$ & & & & $1.7 \mathrm{E}+3$ & 0.17 & $7.1 \mathrm{E}-5$ & $9.7 \mathrm{E}+3$ \\
\hline $\begin{array}{l}\text { EFSA report on } \\
\text { pyridate }\end{array}$ & pyridafol & $\begin{array}{l}\text { aromatic halide } \\
\text { photohydrolysis }\end{array}$ & 3.97 & $1.8 \mathrm{E}-6$ & $3.8 \mathrm{E}+5$ & 1.00 & $2.3 \mathrm{E}-6$ & $3.0 \mathrm{E}+5$ & $3.4 \mathrm{E}+5$ & 0.12 & $2.4 \mathrm{E}-7$ & $2.8 \mathrm{E}+6$ \\
\hline $\begin{array}{l}\text { EFSA report on } \\
\text { pyriproxyfen/pyriproxi } \\
\text { fen }\end{array}$ & pyriproxyfen & $\begin{array}{l}\text { aromatic ether } \\
\text { photohydrolysis }\end{array}$ & 2.29 & $7.0 \mathrm{E}-7$ & $1.0 \mathrm{E}+6$ & & & & $1.0 \mathrm{E}+6$ & 0.57 & $4.0 \mathrm{E}-7$ & $1.7 \mathrm{E}+6$ \\
\hline $\begin{array}{l}\text { EFSA report on } \\
\text { pyroxsulam }\end{array}$ & pyroxsulam & $\begin{array}{l}\text { sulfonamide } \\
\text { photohydrolysis }\end{array}$ & 4.90 & $2.0 \mathrm{E}-6$ & $3.5 \mathrm{E}+5$ & 1.00 & $2.5 \mathrm{E}-6$ & $2.8 \mathrm{E}+5$ & $3.1 \mathrm{E}+5$ & 0.38 & $8.3 \mathrm{E}-7$ & $8.3 \mathrm{E}+5$ \\
\hline $\begin{array}{l}\text { EFSA report on } \\
\text { quizalofop-p-ethyl }\end{array}$ & quizalofop-p-ethyl & $\begin{array}{l}\text { diphenyl ether } \\
\text { photohydrolysis }\end{array}$ & 2.83 & $7.4 \mathrm{E}-8$ & $9.4 \mathrm{E}+6$ & 1.00 & $6.7 \mathrm{E}-7$ & $1.0 \mathrm{E}+6$ & $5.2 \mathrm{E}+6$ & 0.08 & $1.0 \mathrm{E}-8$ & $6.9 \mathrm{E}+7$ \\
\hline $\begin{array}{l}\text { EFSA report on } \\
\text { quizalofop-p-ethyl }\end{array}$ & quizalofop-p-ethyl & $\begin{array}{l}\text { aromatic ether } \\
\text { photohydrolysis }\end{array}$ & 2.83 & $7.4 \mathrm{E}-8$ & $9.4 \mathrm{E}+6$ & 1.00 & $6.7 \mathrm{E}-7$ & $1.0 \mathrm{E}+6$ & $5.2 \mathrm{E}+6$ & 0.03 & $4.3 \mathrm{E}-9$ & $1.6 \mathrm{E}+8$ \\
\hline $\begin{array}{l}\text { EFSA report on } \\
\text { terbuthylazine }\end{array}$ & terbuthylazine & $\begin{array}{l}\text { aromatic halide } \\
\text { photohydrolysis }\end{array}$ & 1.64 & $3.5 \mathrm{E}-7$ & $2.0 \mathrm{E}+6$ & 1.00 & $2.7 \mathrm{E}-7$ & $2.5 \mathrm{E}+6$ & $2.3 \mathrm{E}+6$ & 0.48 & $1.5 \mathrm{E}-7$ & $4.7 \mathrm{E}+6$ \\
\hline $\begin{array}{l}\text { EFSA report on } \\
\text { terbuthylazine }\end{array}$ & terbuthylazine & $\begin{array}{l}\text { s-triazine side chain } \\
\text { photochemical N- } \\
\text { dealkylation }\end{array}$ & 1.64 & $3.5 \mathrm{E}-7$ & $2.0 \mathrm{E}+6$ & 1.00 & $2.7 \mathrm{E}-7$ & $2.5 \mathrm{E}+6$ & $2.3 \mathrm{E}+6$ & 0.14 & $4.3 \mathrm{E}-8$ & $1.6 \mathrm{E}+7$ \\
\hline $\begin{array}{l}\text { EFSA report on } \\
\text { tralkoxydim }\end{array}$ & tralkoxydim & $\begin{array}{l}\text { cyclohexanedione } \\
\text { oxime N-O } \\
\text { photocleavage }\end{array}$ & 1.00 & $1.4 \mathrm{E}-6$ & $5.0 \mathrm{E}+5$ & 1.00 & $1.4 \mathrm{E}-6$ & $5.0 \mathrm{E}+5$ & $5.0 \mathrm{E}+5$ & 0.28 & $3.9 \mathrm{E}-7$ & $1.8 \mathrm{E}+6$ \\
\hline
\end{tabular}




\begin{tabular}{|c|c|c|c|c|c|c|c|c|c|c|c|c|}
\hline reference & contaminant & reaction scheme & $\beta_{\text {exp }}$ & $\begin{array}{l}k_{\text {pa,exp }} \\
\left(\mathrm{s}^{-1}\right)\end{array}$ & $\begin{array}{l}t_{1 / 2, \mathrm{pa}, \exp } \\
(\mathrm{s})\end{array}$ & $\beta_{\text {mod }}$ & $\begin{array}{l}k_{\text {pa,mod }} \\
\left(\mathrm{s}^{-1}\right)\end{array}$ & $\begin{array}{l}t_{1 / 2, \text { pa,mod }} \\
(\mathrm{s})\end{array}$ & $\begin{array}{l}t_{1 / 2, \mathrm{pa}, \mathrm{avg}} \\
(\mathrm{s})\end{array}$ & $\alpha$ & $\begin{array}{l}k_{\text {pr,avg }} \\
\left(\mathrm{s}^{-1}\right)\end{array}$ & $\begin{array}{l}t_{1 / 2, \mathrm{pr} \text {,avg }} \\
(\mathrm{s})\end{array}$ \\
\hline $\begin{array}{l}\text { EFSA report on } \\
\text { metribuzin }\end{array}$ & metribuzin & $\begin{array}{l}\text { 1_2_4-triazine-5-one } \\
\text { photochemical N- } \\
\text { deamination }\end{array}$ & 1.00 & $4.4 \mathrm{E}-5$ & $1.6 \mathrm{E}+4$ & & & & $1.6 \mathrm{E}+4$ & 0.75 & $3.4 \mathrm{E}-5$ & $2.0 \mathrm{E}+4$ \\
\hline $\begin{array}{l}\text { EFSA report on } \\
\text { metribuzin }\end{array}$ & metribuzin & $\begin{array}{l}\text { 1_2_4-triazine-5-one } \\
\text { photochemical N- } \\
\text { deamination }\end{array}$ & & & & 1.00 & $1.2 \mathrm{E}-4$ & $5.9 \mathrm{E}+3$ & $1.6 \mathrm{E}+4$ & 0.80 & $3.4 \mathrm{E}-5$ & $2.0 \mathrm{E}+4$ \\
\hline
\end{tabular}

11

12 Note: Data are for all studies with a valid $\alpha$ value. $k_{\mathrm{pa} \text {,exp }}\left(\right.$ or $\left.k_{\mathrm{pa} \text {,mod }}\right)$ and $t_{1 / 2, \mathrm{pa} \text {,exp }}\left(\right.$ or $\left.t_{1 / 2, \mathrm{pa} \text {,mod }}\right)$ are parent degradation kinetics

13 parameters calculated from the original data reported in the individual reference and our estimated standardization factor $\beta_{\exp }$ (or $\beta_{\text {mod) }}$.

14 The subscript "exp" means that the original data was from an experiment, and "mod" means that the original data was converted to

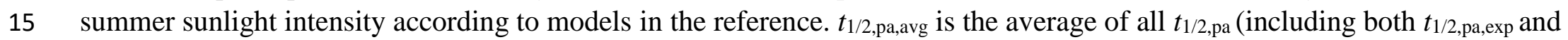

$\left.16 t_{1 / 2, \mathrm{pa} \text {,mod }}\right)$ of the same parent contaminants from different studies. Some referenced studies in the table only have information on the

17 formation ratio $\alpha$ but the parent degradation information can be found from other studies on the same parent compound $\left(t_{1 / 2}\right.$,pa,avg).

$18 t_{1 / 2, \mathrm{pr} \text {,avg }}$ is a product formation parameter calculated as the average of all $t_{1 / 2, \mathrm{pa} \text { avg }} / \alpha$ of the same parent contaminant and the same

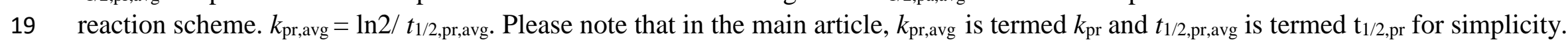

20 The smiles string of the contaminants can be found in the excel file associated with the supporting information. The reference from the

21 European Food Safety Authority (EFSA) reports can be found on the official website and was not referred individually:

22 http://www.efsa.europa.eu/en/publications (accessed on Nov 15, 2020). 
Table S3. Rank levels of all reaction schemes in the direct photolysis reaction library v1.3 and the supporting statistics.

\begin{tabular}{|c|c|c|c|c|c|c|}
\hline$\#$ & reaction scheme name & rank & $\mathrm{n}^{\mathrm{a}}$ & $\mathrm{GM}^{\mathrm{b}}$ of $\mathrm{t}_{1 / 2, \mathrm{pr}}(\mathrm{s})$ & $\mathrm{GSD}^{\mathrm{c}}$ & note $^{\mathrm{d}}$ \\
\hline \multicolumn{7}{|c|}{ Photorearrangement } \\
\hline 1 & 1-Naphthoxy Photorearrangement (C2) & 4 & 1 & & & \\
\hline 2 & 1-Naphthoxy Photorearrangement (C4) & 4 & 1 & & & \\
\hline 3 & 2-Naphthoxy Photorearrangement (C1) & 4 & & & & \\
\hline 4 & 2-Nitrobenzaldehyde Photorearrangement & 4 & 1 & & & \\
\hline 5 & Benzyl Phenyl Ether Photorearrangement (o) & 3 & 1 & & & $2, \mathrm{a}$ \\
\hline 6 & Benzyl Phenyl Ether Photorearrangement (p) & 3 & 1 & & & $2, \mathrm{a}$ \\
\hline 7 & Enone Steroid Photorearrangement to Cyclopentenone & 4 & & & & \\
\hline 8 & Enone Steroid Photorearrangement to Lumiketone & 4 & & & & \\
\hline 9 & O-aryl Carbamate Photorearrangement (o) & 4 & & & & \\
\hline 10 & O-aryl Carbamate Photorearrangement $(\mathrm{p})$ & 4 & & & & \\
\hline 11 & Organothiophosphorus Ester Photochemical Oxygen Transfer & 4 & 1 & & & \\
\hline 12 & Organothiophosphorus Ester Photorearrangement & 4 & & & & \\
\hline 13 & Phenoxyphenol Dehalogenative Photorearrangement & 4 & 1 & & & $2, \mathrm{~b}$ \\
\hline \multicolumn{7}{|c|}{ Photodissociation } \\
\hline 14 & Aromatic Ketone Norrish II Photocleavage (C1_C4) & 4 & 1 & & & \\
\hline 15 & Aminobenzophenone Photochemical N-dealkylation & 4 & & & & \\
\hline 16 & Benzyl Photodeamination to Alcohol & 3 & 1 & & & $2, \mathrm{a}$ \\
\hline 17 & Benzyl Photodeamination to Carbonyl & 3 & 1 & & & $2, \mathrm{a}$ \\
\hline 18 & Benzyl Thiocarbamate Photocleavage to Carbonyl & 2 & 2 & $6.6 \mathrm{E}+06$ & 0.4 & $1, \mathrm{a}$ \\
\hline 19 & Cyclohexanedione Oxime N-O Photocleavage & 4 & & & & \\
\hline 20 & Diazepam Ring Photocleavage & 3 & 2 & $1.7 \mathrm{E}+06$ & 0.2 & 1 \\
\hline 21 & Dihydrophenanthrene Benzyl Photodealkylation & 4 & & & & \\
\hline 22 & Dihydrophenanthrene Benzyl Oxidative Photodealkylation & 4 & & & & \\
\hline 23 & Dinitroaniline Photochemical N-dealkylation & 4 & 1 & & & \\
\hline 24 & Fluoroquinolone Ethylenediamine Photochemical N-dealkylation & 7 & & & & $2, \mathrm{e}$ \\
\hline 25 & Fluoroquinolone Photochemical N-dealkylation & 6 & 2 & $1.8 \mathrm{E}+03$ & 0.2 & $1, \mathrm{e}$ \\
\hline
\end{tabular}




\begin{tabular}{|c|c|c|c|c|c|c|}
\hline \# & reaction scheme name & rank & $\mathrm{n}^{\mathrm{a}}$ & $\mathrm{GM}^{\mathrm{b}}$ of $\mathrm{t}_{1 / 2, \mathrm{pr}}(\mathrm{s})$ & $\mathrm{GSD}^{\mathrm{c}}$ & note \\
\hline 26 & Fluoroquinolone Piperazine Photochemical Bis N-dealkylation & 7 & 1 & & & $2, \mathrm{e}$ \\
\hline 27 & Imidazolinone Ring Photocleavage to Aldehyde & 4 & & & & \\
\hline 28 & Imidazolinone Ring Photocleavage to Amide & 4 & & & & \\
\hline 29 & Imidazolinone Ring Photocleavage to Amidine & 4 & & & & \\
\hline 30 & Imidazolinone Ring Photocleavage to Carboxylic Acid & 4 & & & & \\
\hline 31 & Nitroenamine Photocleavage & 4 & & & & \\
\hline 32 & Nitroenamine Photocleavage to Carbonyl & 4 & & & & \\
\hline 33 & Nitrosamine N-C Photocleavage & 4 & 1 & & & \\
\hline 34 & p-Aminobenzoic Acid Photochemical N-dealkylation & 4 & & & & \\
\hline 35 & Phenoxyphenol Ether Photocleavage & 4 & 1 & & & $2, \mathrm{~b}$ \\
\hline 36 & Phenylurea Photochemical N-dealkylation & 1 & 1 & & & $2, \mathrm{c}$ \\
\hline 37 & Phenylurea Photochemical N-demethoxylation & 1 & 1 & & & $2, \mathrm{c}$ \\
\hline 38 & Phenylurea N-formyl Photocleavage & 4 & & & & \\
\hline 39 & Pyridinium Photochemical N-dealkylation & 4 & & & & \\
\hline 40 & s-Triazine Side Chain Photochemical N-dealkylation & 3 & 4 & $4.2 \mathrm{E}+06$ & 1.3 & 1 \\
\hline 41 & Sulfonamide N-C Photocleavage (6-5) & 4 & & & & \\
\hline 42 & Tetracycline Photochemical N-dealkylation & 4 & & & & \\
\hline \multicolumn{7}{|c|}{ Photoelimination } \\
\hline 43 & 1_2_4-Triazine-5-one Photochemical N-deamination & 4 & & & & \\
\hline 44 & Aromatic Acetic Acid Photodecarboxylation & 4 & 3 & $1.6 \mathrm{E}+05$ & 1.1 & 1 \\
\hline 45 & Aromatic Acetic Acid Photodecarboxylation to Alcohol & 4 & 1 & & & \\
\hline 46 & Aromatic Acetic Acid Photodecarboxylation to Carbonyl & 4 & 5 & $4.6 \mathrm{E}+05$ & 1.6 & 1 \\
\hline 47 & Aromatic Carboxylic Acid Photodecarboxylation & 4 & 1 & & & \\
\hline 48 & Aromatic Carboxylic Acid Photodecarboxylation to Alcohol & 4 & 1 & & & \\
\hline 49 & Benzotriazole Photodenitrogenation & 4 & & & & \\
\hline 50 & Benzotriazole Photodenitrogenation to Phenol (o) & 4 & & & & \\
\hline 51 & Cephem Photodecarboxylation & 4 & & & & \\
\hline 52 & Cyanohydrin Cyano Photoelimination to Aldehyde & 4 & & & & \\
\hline 53 & Fipronil Sulfoxide Photoextrusion & 4 & & & & \\
\hline
\end{tabular}




\begin{tabular}{|c|c|c|c|c|c|c|}
\hline$\#$ & reaction scheme name & rank & $\mathrm{n}^{\mathrm{a}}$ & $\mathrm{GM}^{\mathrm{b}}$ of $\mathrm{t}_{1 / 2, \mathrm{pr}}(\mathrm{s})$ & $\mathrm{GSD}^{\mathrm{c}}$ & note $^{\mathrm{d}}$ \\
\hline 54 & Imidazolinone Amide Photoelimination & 4 & & & & \\
\hline 55 & Imidazolinone Photodecarbonylation & 4 & & & & \\
\hline 56 & Nitroguanidine Photochemical N-denitration & 4 & 1 & & & \\
\hline 57 & Nitrosamine N-N Photocleavage & 4 & 1 & & & \\
\hline 58 & Phenoxyacetic Acid Photodecarboxylation & 4 & & & & \\
\hline 59 & Phenoxyacetic Acid Photodecarboxylation to Carbonyl & 4 & & & & \\
\hline 60 & Pyrrolinone Photodecarbonylation & 4 & & & & \\
\hline 61 & RDX Photochemical N-denitration to Imine & 4 & & & & \\
\hline 62 & Sulfonamide SO2 Extrusion Photorearrangement (6-6) & 4 & 1 & & & $2, \mathrm{~d}$ \\
\hline \multicolumn{7}{|c|}{ Photocyclization } \\
\hline 63 & Acetanilide Dehalogenative Photocyclization to Pyrrolinone & 4 & & & & \\
\hline 64 & $\begin{array}{l}\text { Acetanilide O-dealkyl Dehalogenative Photocyclization to } \\
\text { Morpholinone }\end{array}$ & 4 & & & & \\
\hline 65 & Altrenogest Photocycloaddition & 7 & 1 & & & 3 \\
\hline 66 & Aminobenzophenone Photocyclization to Acridinone & 4 & & & & \\
\hline 67 & Anthranilic Diamide Dehalogenative Photocyclization to Oxazine & 4 & 1 & & & \\
\hline 68 & Aromatic Ketone Norrish II Photocyclization (C1_C4) & 4 & 1 & & & \\
\hline 69 & beta-Triketone Dehalogenative Photocyclization to Pyran & 4 & 1 & & & \\
\hline 70 & Diarylethene Photocyclization to Phenanthrene & 4 & & & & \\
\hline 71 & Diarylethene Photocyclization to Phenanthrene (E isomer) & 4 & & & & \\
\hline 72 & Dinitroaniline Photocyclization to Benzimidazole (NOHOH) & 4 & & & & \\
\hline 73 & $\begin{array}{l}\text { Dinitroaniline Photocyclization to Benzimidazole (NOHOH to } \\
\text { NO) }\end{array}$ & 7 & & & & 4 \\
\hline 74 & Dinitroaniline Photocyclization to Benzimidazole (NO to N) & 4 & & & & \\
\hline 75 & Diphenylamine Photocyclization to Carbazole & 4 & & & & \\
\hline 76 & Diphenylamine Dehalogenative Photocyclization to Carbazole & 4 & 1 & & & \\
\hline 77 & Fluoroquinolone Defluorinative Photocyclization & 7 & & & & $2, \mathrm{e}$ \\
\hline 78 & Lamotrigine Photocyclization to Carbazole & 4 & & & & \\
\hline 79 & Lamotrigine Dehalogenative Photocyclization to Carbazole & 4 & & & & \\
\hline
\end{tabular}




\begin{tabular}{|c|c|c|c|c|c|c|}
\hline \# & reaction scheme name & rank & $\mathrm{n}^{\mathrm{a}}$ & $\mathrm{GM}^{\mathrm{b}}$ of $\mathrm{t}_{1 / 2, \mathrm{pr}}(\mathrm{s})$ & $\mathrm{GSD}^{\mathrm{c}}$ & note \\
\hline 80 & o-Vinylbiphenyl Photocyclization to Dihydrophenanthrene & 5 & 2 & $7.4 \mathrm{E}+04$ & 0.1 & 1 \\
\hline 81 & Phenoxyphenol Dehalogenative Photocyclization to Dioxin & 4 & 5 & $2.0 \mathrm{E}+05$ & 0.4 & $1, \mathrm{~b}$ \\
\hline \multicolumn{7}{|c|}{ Photochemical Ring Contraction } \\
\hline 82 & Zepine Photochemical Ring Contraction to Acridine & 4 & & & & \\
\hline \multicolumn{7}{|c|}{ Photohydrolysis } \\
\hline 83 & Aromatic Amine Photohydrolysis & 4 & 1 & & & \\
\hline 84 & Aromatic Carbamate Photohydrolysis & 4 & 1 & & & \\
\hline 85 & Aromatic Ether Photohydrolysis & 3 & 8 & $1.1 \mathrm{E}+06$ & 1.1 & 1 \\
\hline 86 & Aromatic Halide Photohydrolysis & 3 & 14 & $1.4 \mathrm{E}+06$ & 1.4 & 1 \\
\hline 87 & Aromatic Nitro Photohydrolysis & 3 & 3 & $6.2 \mathrm{E}+05$ & 1.5 & 1 \\
\hline 88 & Aromatic Sulfonate Photohydrolysis & 4 & & & & \\
\hline 89 & Benzoylphenylurea Amide Photohydrolysis & 4 & & & & \\
\hline 90 & Benzoylphenylurea Urea Photohydrolysis & 4 & & & & \\
\hline 91 & beta-Triketone alpha Photocleavage to Carboxylic Acid & 4 & 1 & & & \\
\hline 92 & Diphenyl Ether Photohydrolysis & 4 & 1 & & & \\
\hline 93 & Fluoroquinolone Fluoride Photohydrolysis & 7 & 1 & & & $2, \mathrm{e}$ \\
\hline 94 & N-aryl Amide Photohydrolysis & 3 & 2 & $2.6 \mathrm{E}+06$ & 1.3 & 1 \\
\hline 95 & Nitrofuran Imine Photohydrolysis & 4 & & & & \\
\hline 96 & Nitroguanidine Imine Photohydrolysis & 4 & 2 & $1.7 \mathrm{E}+05$ & 0.3 & 1 \\
\hline 97 & Nitroguanidine Nitro Photohydrolysis & 4 & 1 & & & 3 \\
\hline 98 & Organophosphorus Ester Photohydrolysis & 3 & 5 & $1.1 \mathrm{E}+06$ & 1.4 & 1 \\
\hline 99 & Pyrethroid Carboxylic Acid Ester Photohydrolysis & 4 & 1 & & & \\
\hline 100 & Pyrrolinone Halide Photohydrolysis & 4 & & & & \\
\hline 101 & Sulfonamide Photohydrolysis & 4 & 6 & $2.3 \mathrm{E}+05$ & 0.7 & $1, \mathrm{~d}$ \\
\hline 102 & Sulfonamide S-C Photohydrolysis & 3 & 2 & $1.5 \mathrm{E}+06$ & 0.4 & $1, \mathrm{~d}$ \\
\hline 103 & Sulfonylurea Photohydrolysis & 4 & & & & \\
\hline 104 & Sulfonylurea S-C Photohydrolysis & 4 & & & & \\
\hline 105 & Sulfonylurea S-N Photohydrolysis & 4 & & & & \\
\hline 106 & Trifluoromethyl Photohydrolysis & 4 & 1 & & & \\
\hline
\end{tabular}




\begin{tabular}{|c|c|c|c|c|c|c|}
\hline$\#$ & reaction scheme name & rank & $\mathrm{n}^{\mathrm{a}}$ & $\mathrm{GM}^{\mathrm{b}}$ of $\mathrm{t}_{1 / 2, \mathrm{pr}}(\mathrm{s})$ & $\mathrm{GSD}^{\mathrm{c}}$ & note $^{\mathrm{d}}$ \\
\hline \multicolumn{7}{|c|}{ Photohydration } \\
\hline 107 & Diarylethene Photohydration & 5 & 2 & $8.1 \mathrm{E}+04$ & 0.1 & 1 \\
\hline 108 & Dienone Steroid Photohydration (C5) & 4 & & & & \\
\hline 109 & Dienone Steroid Photohydration (C9) & 4 & 1 & & & \\
\hline 110 & Enone Steroid Photohydration and Photorearrangement to Spiro & 4 & & & & \\
\hline 111 & Trienone Steroid Photohydration (C10) & 4 & & & & \\
\hline 112 & Trienone Steroid Photohydration (C12) & 4 & 1 & & & \\
\hline \multicolumn{7}{|c|}{ Photooxidation } \\
\hline 113 & 1_2-Naphthoquinone Photohydroxylation (C4) & 4 & & & & \\
\hline 114 & 1_4-Naphthoquinone Photohydroxylation (C5) & 4 & & & & \\
\hline 115 & 1_4-Naphthoquinone Photohydroxylation (C6) & 4 & & & & \\
\hline 116 & 1-Hydroxypyrene Photooxidation to Quinone (C1_C6) & 4 & & & & \\
\hline 117 & 1-Hydroxypyrene Photooxidation to Quinone (C1_C8) & 4 & & & & \\
\hline 118 & 1-Naphthol Photooxidation to 1_2-Benzoquinone & 4 & & & & \\
\hline 119 & 1-Naphthol Photooxidation to 1_4-Benzoquinone & 4 & & & & \\
\hline 120 & 1-Naphthoxy Oxidative Photocleavage to 1_4-Benzoquinone & 4 & & & & \\
\hline 121 & Anthracene Photooxidation to Endoperoxide & 4 & & & & \\
\hline 122 & Aromatic Methyl Photooxidation to Carboxylic Acid & 4 & 1 & & & \\
\hline 123 & Aromatic Nitroso Photooxidation & 4 & 1 & & & \\
\hline 124 & Aromatic Sulfoxide Photooxidation & 4 & & & & \\
\hline 125 & Aromatic Thioether Photooxidation & 4 & 3 & $3.3 \mathrm{E}+05$ & 0.3 & 1 \\
\hline 126 & Benzaldehyde Photooxidation to Carboxylic Acid & 4 & & & & \\
\hline 127 & Benzyl Thio Photooxidation to Sulfoxide & 2 & 2 & $5.9 \mathrm{E}+06$ & 0.2 & $1, \mathrm{a}$ \\
\hline 128 & beta-Triketone alpha Photohydroxylation (Dienol) & 4 & & & & \\
\hline 129 & beta-Triketone alpha Photohydroxylation (Keto) & 4 & & & & \\
\hline 130 & beta-Triketone Photohydroxylation (Enol) & 4 & & & & \\
\hline 131 & beta-Triketone Photohydroxylation (Keto) & 4 & & & & \\
\hline 132 & Carbamazepine Photoepoxidation & 4 & 1 & & & \\
\hline 133 & Diarylethene Photooxidation & 4 & 1 & & & \\
\hline
\end{tabular}




\begin{tabular}{|c|c|c|c|c|c|c|}
\hline \# & reaction scheme name & rank & $\mathrm{n}^{\mathrm{a}}$ & $\mathrm{GM}^{\mathrm{b}}$ of $\mathrm{t}_{1 / 2, \mathrm{pr}}(\mathrm{s})$ & $\mathrm{GSD}^{\mathrm{c}}$ & note $^{\mathrm{d}}$ \\
\hline 134 & Dihydrooxathiine Anilide Photooxidation to Sulfoxide & 4 & 1 & & & \\
\hline 135 & Dihydropyridine Photooxidation to Pyridine & 4 & 1 & & & \\
\hline 136 & Octahydrophenanthrene Benzyl Photohydroxylation & 4 & & & & \\
\hline 137 & Octahydrophenanthrene Benzyl Photooxidation to Ketone & 4 & & & & \\
\hline 138 & Organothiophosphorus Ester Photooxidation to Oxon & 3 & 4 & $1.1 \mathrm{E}+06$ & 1.3 & 1 \\
\hline 139 & Phenylurea N-methyl Photooxidation to N-formyl & 1 & 1 & & & $2, \mathrm{c}$ \\
\hline 140 & Pyrene Aromatic Photohydroxylation & 4 & & & & \\
\hline 141 & s-Triazine Side Chain N-alkyl Photooxidation to Carbonyl & 3 & 3 & $3.1 \mathrm{E}+06$ & 1.6 & 1 \\
\hline 142 & s-Triazine Side Chain N-isopropyl Photooxidation to Ketone & 2 & 2 & $2.6 \mathrm{E}+07$ & 0.3 & 1 \\
\hline 143 & Trienone Steroid Photooxidation to Dialdehyde & 4 & 1 & & & \\
\hline 144 & Trifluoroacetic Acid Photoformation & 4 & & & & \\
\hline \multicolumn{7}{|c|}{ Photoreduction } \\
\hline 145 & Aromatic Photohydrodehalogenation & 2 & 10 & $6.2 \mathrm{E}+06$ & 0.6 & 1 \\
\hline 146 & Dinitroaniline Nitro Photoreduction & 4 & & & & \\
\hline 147 & Fluoroquinolone Photohydrodefluorination & 7 & & & & $2, \mathrm{e}$ \\
\hline \multicolumn{7}{|c|}{ Secondary Dark Reaction } \\
\hline 148 & 12-OH Steroid Dehydration to Trienone & 4 & & & & \\
\hline 149 & 5-OH Steroid Dehydration to Dienone & 4 & & & & \\
\hline 150 & Aldehyde Oxidation to Carboxylic Acid & 4 & & & & \\
\hline 151 & C-NCO Hydrolysis & 4 & & & & \\
\hline 152 & C-NNO2 Hydrolysis & 4 & & & & \\
\hline 153 & Dehydration of Geminal Diols & 7 & & & & 4 \\
\hline 154 & Hydroxy Enal Tautomerization & 4 & & & & \\
\hline 155 & Nitro Amidine Hydrolysis & 4 & & & & \\
\hline
\end{tabular}

24 Note: ${ }^{a} \mathrm{n}$ stands for the count of data points on $t_{1 / 2, \mathrm{pr}}$ for the same reaction scheme. ${ }^{\mathrm{b}} \mathrm{GM}$ stands for geometric mean. ${ }^{\mathrm{c}} \mathrm{GSD}$ stands for

25 geometric standard deviation. 'in the "note" column, " 1 " marks the reaction schemes that have at least two data points on $t_{1 / 2, \text { pr }}$ and the

26 rank levels were assigned based on their GM, " 2 " marks the reaction schemes that have one or no data points on $t_{1 / 2, \text { pr }}$ but the rank levels

27 were assigned according to the GM of data points of relevant reaction schemes which are notated with the same letter (a, b, c, d, or e), 
“3” marks specific reaction schemes whose rank levels were assigned based on a single data point on $t_{1 / 2, \mathrm{pr}}$, and " 4 " marks

29 spontaneous reaction schemes whose rank levels were assigned to be the highest level (7). 
Table S4. Internal evaluation of each version of the direct photolysis library against DB-J-ENV revealing step-wise improvement.

\begin{tabular}{|c|c|c|c|c|c|c|c|c|c|}
\hline \multirow[b]{2}{*}{ generation } & \multicolumn{5}{|c|}{ All products } & \multirow[b]{2}{*}{$\begin{array}{c}\text { step-wise \% } \\
\text { change in } \\
\text { recall }^{c}\end{array}$} & \multirow[b]{2}{*}{$\begin{array}{c}\text { step-wise \% } \\
\text { change in } \\
\text { precision }^{c}\end{array}$} & \multicolumn{2}{|c|}{$\begin{array}{l}\text { major } \\
\text { products }\end{array}$} \\
\hline & $\mathrm{N}_{\mathrm{O}, \mathrm{i}}$ & $\mathrm{N}_{\mathrm{OP}, \mathrm{i}}^{\mathrm{a}}$ & $\mathrm{N}_{P, \mathrm{i}}$ & recall $^{\mathrm{a}}$ & precision $^{\mathrm{a}}$ & & & $\mathrm{N}_{\mathrm{O}, \mathrm{i}}$ & $\mathrm{N}_{\mathrm{OP}, \mathrm{i}}^{\mathrm{b}}$ \\
\hline \multicolumn{10}{|c|}{ v1.0 (original library) } \\
\hline 1 & 891 & 563 & 1608 & 0.40 & 0.37 & & & 177 & 142 \\
\hline $1+2$ & 1241 & 676 & 4341 & 0.48 & 0.16 & & & & \\
\hline $1+2+3$ & 1353 & 703 & 7775 & 0.50 & 0.09 & & & & \\
\hline all & 1396 & & & & & & & & \\
\hline \multicolumn{10}{|c|}{$\mathrm{v} 1.1(\mathrm{v} 1.0+$ relative reasoning rules from DB-J-ENV) } \\
\hline 1 & $n c^{d}$ & nc & 1541 & nc & 0.37 & 0 & 4.3 & nc & 142 \\
\hline $1+2$ & nc & nc & 4161 & nc & 0.16 & 0 & 4.3 & & \\
\hline $1+2+3$ & nc & nc & 7425 & nc & 0.09 & 0 & 4.7 & & \\
\hline \multicolumn{10}{|c|}{$\mathrm{v} 1.2(\mathrm{v} 1.1+$ additional relative reasoning rules from DB-EFSA-ENV) } \\
\hline 1 & nc & nc & 1533 & nc & 0.37 & 0 & 0.5 & nc & 142 \\
\hline $1+2$ & nc & nc & 4142 & nc & 0.16 & 0 & 0.5 & & \\
\hline $1+2+3$ & nc & nc & 7400 & nc & 0.10 & 0 & 0.3 & & \\
\hline \multicolumn{10}{|c|}{ v1.3 (v1.2 + ranking from DB-J-ENV) } \\
\hline 1 & nc & 531 & 1319 & 0.38 & 0.40 & -5.7 & 9.6 & nc & 139 \\
\hline $1+2$ & nc & 636 & 3241 & 0.46 & 0.20 & -5.9 & 20.2 & & \\
\hline $1+2+3$ & nc & 661 & 5447 & 0.47 & 0.12 & -6.0 & 27.7 & & \\
\hline
\end{tabular}

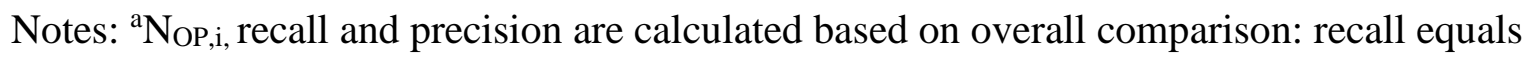
$\mathrm{N}_{\mathrm{OP}, \mathrm{i}} / \mathrm{N}_{\mathrm{O} \_}$all , and precision equals $\mathrm{N}_{\mathrm{OP}, \mathrm{i}} / \mathrm{N}_{\mathrm{P}, \mathrm{i}} .{ }^{\mathrm{b}}$ major products are calculated based on firstgeneration comparison. ${ }^{c}$ compared to the above library version (different from Table 3 in the main article) and the value of the above library version is used as the denominator. ${ }^{\mathrm{d}}$ Numbers that should not be changed from the original library. 
Table S5. External evaluation of each version of the direct photolysis library against DB-EFSAENV revealing step-wise improvement.

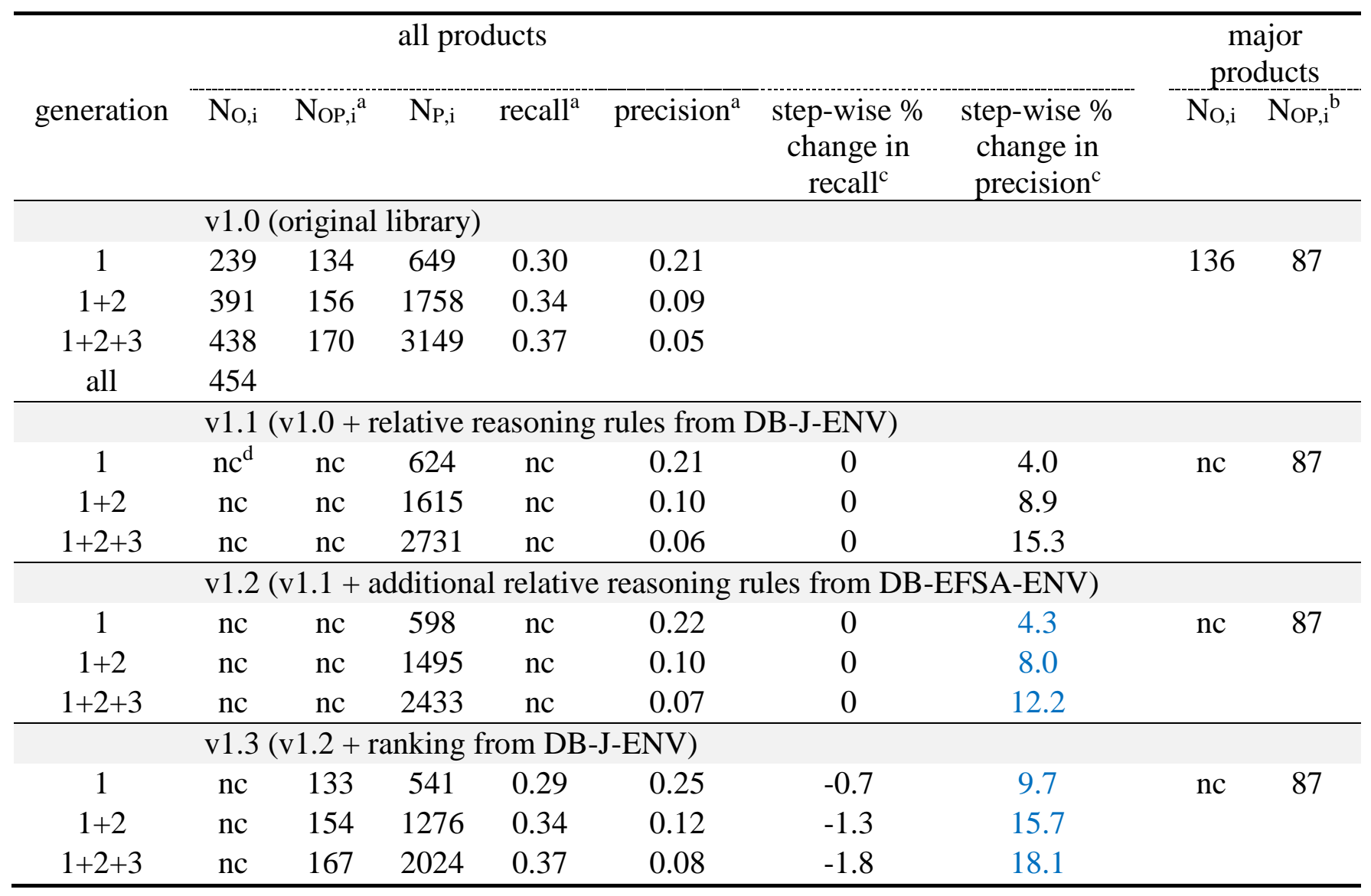

Notes: ${ }^{a} \mathrm{~N}_{\mathrm{OP}, \mathrm{i}}$, recall and precision are calculated based on overall comparison: recall equals $\mathrm{N}_{\mathrm{OP}, \mathrm{i}} / \mathrm{N}_{\mathrm{O} \_ \text {all }}$, and precision equals $\mathrm{N}_{\mathrm{OP}, \mathrm{i}} / \mathrm{N}_{\mathrm{P}, \mathrm{i} .}{ }^{\mathrm{b}}$ major products are calculated based on firstgeneration comparison. ${ }^{c}$ compared to the above library version (different from Table 4 in the main article) and the value of the above library version is used as the denominator. ${ }^{\mathrm{d}}$ Numbers that should not be changed from the original library. 
Table S6. Improvement of the evaluation measures after modification of "trifluoromethyl photohydrolysis" for data in DB-J-ENV.

\begin{tabular}{|c|c|c|c|c|c|c|c|c|c|}
\hline \multirow[b]{2}{*}{ generation } & \multicolumn{5}{|c|}{ all products } & \multirow[b]{2}{*}{$\begin{array}{l}\text { step-wise \% } \\
\text { change in } \\
\text { recall }^{c}\end{array}$} & \multirow[b]{2}{*}{$\begin{array}{l}\text { step-wise \% } \\
\text { change in } \\
\text { precision }^{\mathrm{c}}\end{array}$} & \multicolumn{2}{|c|}{$\begin{array}{c}\text { major } \\
\text { products }\end{array}$} \\
\hline & $\mathrm{N}_{\mathrm{O}, \mathrm{i}}$ & $\mathrm{N}_{\mathrm{OP}, \mathrm{i}^{\mathrm{a}}}$ & $\mathrm{N}_{\mathrm{P}, \mathrm{i}}$ & recall $^{\mathrm{a}}$ & precision $^{\mathrm{a}}$ & & & $\mathrm{N}_{\mathrm{O}, \mathrm{i}}$ & $\mathrm{N}_{\mathrm{OP}, \mathrm{i}^{\mathrm{b}}}$ \\
\hline & \multicolumn{7}{|c|}{$\mathrm{v} 1.2$ (v1.1 + additional relative reasoning rules from DB-EFSA-ENV) } & & \\
\hline 1 & 891 & 563 & 1533 & 0.40 & 0.37 & & & 177 & 142 \\
\hline $1+2$ & 1241 & 676 & 4142 & 0.48 & 0.16 & & & & \\
\hline \multirow[t]{2}{*}{$1+2+3$} & 1353 & 703 & 7400 & 0.50 & 0.10 & & & & \\
\hline & \multicolumn{7}{|c|}{$\mathrm{v} 1.2^{+}$(v1.2 + additional rules for "trifluomethyl photohydrolysis") } & & \\
\hline 1 & $\mathrm{nc}^{\mathrm{d}}$ & 561 & 1529 & 0.40 & 0.37 & -0.4 & -0.1 & $\mathrm{nc}$ & 142 \\
\hline $1+2$ & $\mathrm{nc}$ & 673 & 4118 & 0.48 & 0.16 & -0.4 & 0.1 & & \\
\hline \multirow[t]{2}{*}{$1+2+3$} & $\mathrm{nc}$ & 699 & 7332 & 0.50 & 0.10 & -0.6 & 0.4 & & \\
\hline & \multicolumn{7}{|c|}{$\mathrm{v} 1.3$ (v $1.2+$ ranking from DB-J-ENV) } & & \\
\hline 1 & nc & 531 & 1319 & 0.38 & 0.40 & & & $\mathrm{nc}$ & 139 \\
\hline $1+2$ & $\mathrm{nc}$ & 636 & 3241 & 0.46 & 0.20 & & & & \\
\hline \multirow[t]{2}{*}{$1+2+3$} & $\mathrm{nc}$ & 661 & 5447 & 0.47 & 0.12 & & & & \\
\hline & \multicolumn{7}{|c|}{ v1.3+ (v1.3 + additional rules for "trifluomethyl photohydrolysis") } & & \\
\hline 1 & $\mathrm{nc}$ & 529 & 1317 & 0.38 & 0.40 & -0.4 & -0.2 & $\mathrm{nc}$ & 139 \\
\hline $1+2$ & $\mathrm{nc}$ & 633 & 3227 & 0.45 & 0.20 & -0.5 & -0.04 & & \\
\hline $1+2+3$ & $\mathrm{nc}$ & 657 & 5412 & 0.12 & 0.12 & -0.6 & 0.04 & & \\
\hline
\end{tabular}

Notes: ${ }^{a} \mathrm{~N}_{\mathrm{OP}, \mathrm{i}, \text { recall and precision are calculated based on overall comparison: recall equals }}$ $\mathrm{N}_{\mathrm{OP}, \mathrm{i}} / \mathrm{N}_{\mathrm{O} \_a l l}$, and precision equals $\mathrm{N}_{\mathrm{OP}, \mathrm{i}} / \mathrm{N}_{\mathrm{P}, \mathrm{i}} .{ }^{\mathrm{b}}$ major products are calculated based on first generation comparison. ${ }^{c}$ compared to the above library version (e.g. v1.3 ${ }^{+}$is compared to v1.3) and the value of the above library version is used as the denominator. ${ }^{\mathrm{d}}$ Numbers that should not be changed from the original library. 
Table S7. Improvement of evaluation measures after modification of "trifluoromethyl photohydrolysis" for data in DB-EFSA-ENV.

\begin{tabular}{|c|c|c|c|c|c|c|c|c|c|}
\hline \multirow[b]{2}{*}{ generation } & \multicolumn{5}{|c|}{ all products } & \multirow[b]{2}{*}{$\begin{array}{c}\text { step-wise } \% \\
\text { change in }^{\text {recall }}\end{array}$} & \multirow[b]{2}{*}{$\begin{array}{l}\text { step-wise \% } \\
\text { change in } \\
\text { precision }^{\mathrm{c}}\end{array}$} & \multicolumn{2}{|c|}{$\begin{array}{l}\text { major } \\
\text { products }\end{array}$} \\
\hline & $\mathrm{N}_{\mathrm{O}, \mathrm{i}}$ & $\mathrm{N}_{\mathrm{OP}, \mathrm{i}^{\mathrm{a}}}$ & $\mathrm{N}_{\mathrm{P}, \mathrm{i}}$ & recall $^{\mathrm{a}}$ & precision $^{\mathrm{a}}$ & & & $\mathrm{N}_{\mathrm{O}, \mathrm{i}}$ & $\mathrm{NOP}, \mathrm{i}_{\mathrm{b}}^{\mathrm{b}}$ \\
\hline & \multicolumn{7}{|c|}{ v1.2 (v1.1 + additional relative reasoning rules from DB-EFSA-ENV) } & & \\
\hline 1 & 239 & 134 & 598 & 0.30 & 0.22 & & & 136 & 87 \\
\hline $1+2$ & 391 & 156 & 1495 & 0.34 & 0.10 & & & & \\
\hline $1+2+3$ & 438 & 170 & 2433 & 0.37 & 0.07 & & & & \\
\hline \multicolumn{10}{|c|}{$\mathrm{v} 1.2^{+}(\mathrm{v} 1.2+$ additional rules for "trifluomethyl photohydrolysis") } \\
\hline 1 & $n c^{\mathrm{d}}$ & 134 & 589 & 0.30 & 0.23 & 0 & 1.5 & $\mathrm{nc}$ & 87 \\
\hline $1+2$ & $\mathrm{nc}$ & 156 & 1445 & 0.34 & 0.11 & 0 & 3.5 & & \\
\hline $1+2+3$ & $\mathrm{nc}$ & 169 & 2313 & 0.37 & 0.07 & -0.6 & 4.6 & & \\
\hline \multicolumn{10}{|c|}{$\mathrm{v} 1.3(\mathrm{v} 1.2+$ ranking from DB-J-ENV } \\
\hline 1 & $\mathrm{nc}$ & 133 & 541 & 0.29 & 0.25 & & & $\mathrm{nc}$ & 87 \\
\hline $1+2$ & $\mathrm{nc}$ & 154 & 1276 & 0.34 & 0.12 & & & & \\
\hline $1+2+3$ & $\mathrm{nc}$ & 167 & 2024 & 0.37 & 0.08 & & & & \\
\hline \multicolumn{10}{|c|}{ v1.3 ( $3^{+} 1.3+$ additional rules for "trifluomethyl photohydrolysis") } \\
\hline 1 & $\mathrm{nc}$ & 133 & 532 & 0.29 & 0.25 & 0 & 1.7 & $\mathrm{nc}$ & 87 \\
\hline $1+2$ & $\mathrm{nc}$ & 154 & 1234 & 0.34 & 0.12 & 0 & 3.4 & & \\
\hline $1+2+3$ & $\mathrm{nc}$ & 167 & 1945 & 0.37 & 0.09 & 0 & 4.1 & & \\
\hline
\end{tabular}

Notes: ${ }^{\mathrm{a}} \mathrm{N}_{\mathrm{OP}, \mathrm{i}, \text { recall and precision are calculated based on overall comparison: recall equals }}$ $\mathrm{N}_{\mathrm{OP},} / \mathrm{N}_{\mathrm{O} \_a l l}$, and precision equals $\mathrm{N}_{\mathrm{OP}, i} / \mathrm{N}_{\mathrm{P}, \mathrm{i}} .{ }^{\mathrm{b}}$ major products are calculated based on first generation comparison. ${ }^{c}$ compared to the above library version (e.g. v1.3 ${ }^{+}$is compared to v1.3) and the value of the above library version is used as the denominator. ${ }^{\mathrm{d}}$ Numbers that should not be changed from the original library. 
Table S8. List of acronyms.

\begin{tabular}{|c|c|}
\hline & library versions \\
\hline library v1.0 & the original direct photolysis library developed in our previous paper ${ }^{91}$ \\
\hline library v1.1 & library v1.0 plus the relative reasoning rules from DB-J-ENV \\
\hline library v1.2 & library v1.1 plus the relative reasoning rules from DB-EFSA-ENV \\
\hline library v1.3 & library v1.2 with assigned ranks for each reaction scheme \\
\hline library v1.2 $2^{+}$ & $\begin{array}{l}\text { the same as library } 1.2 \text { except that the "trifluoromethyl photohydrolysis" } \\
\text { scheme is upgraded according to the manuscript }\end{array}$ \\
\hline library v1.3 $3^{+}$ & $\begin{array}{l}\text { the same as library } 1.3 \text { except that the "trifluoromethyl photohydrolysis" } \\
\text { scheme is upgraded according to the manuscript }\end{array}$ \\
\hline & abbreviations \\
\hline $\mathrm{N}_{\mathrm{OP}}$ & the number of observed products that are corrected predicted \\
\hline $\mathrm{N}_{\mathrm{O}}$ & the number of observed products \\
\hline $\mathrm{N}_{\mathrm{P}}$ & the number of predicted products \\
\hline NOP_i & the number of observed products that are correctly predicted up to generation $\mathrm{i}$ \\
\hline $\mathrm{N}_{\mathrm{O}_{-} \mathrm{i}}$ & the number of observed products up to generation $\mathrm{i}$ \\
\hline $\mathrm{N}_{\mathrm{O} \_a l l}$ & the number of observed products for all generations \\
\hline $\mathrm{N}_{\mathrm{P} \_\mathrm{i}}$ & the number of predicted products up to generation $\mathrm{i}$ \\
\hline $\mathrm{N}_{\mathrm{OP} \_\mathrm{r}}$ & $\begin{array}{l}\text { the number of parent compounds observed and correctly predicted to undergo } \\
\text { a certain reaction scheme }\end{array}$ \\
\hline $\mathrm{N}_{\mathrm{O}_{-} \mathrm{r}}$ & $\begin{array}{l}\text { the number of parent compounds observed to undergo a certain reaction } \\
\text { scheme }\end{array}$ \\
\hline $\mathrm{N}_{\mathrm{P}_{\mathrm{r}} \mathrm{r}}$ & $\begin{array}{l}\text { the number of parent compounds predicted to undergo a certain reaction } \\
\text { scheme }\end{array}$ \\
\hline$k_{\mathrm{pa}}$ & the first-order rate constant of the parent compound \\
\hline$k_{\mathrm{pr}}$ & $\begin{array}{l}\text { the first-order rate constant of the parent compound to form the product } \\
\text { through a certain reaction scheme }\end{array}$ \\
\hline$t_{1 / 2, \mathrm{pa}}$ & the half-life of the parent compound \\
\hline$t_{1 / 2, \mathrm{pr}}$ & $\begin{array}{l}\text { the reaction-scheme-specific half-life, which is the half-life of the parent } \\
\text { compound to form the product through a certain reaction scheme }\end{array}$ \\
\hline$\alpha$ & $\begin{array}{l}\text { the formation ratio, which is the ratio of the first-order rate constant of the } \\
\text { first-generation reaction scheme to form the product divided by the first-order } \\
\text { rate constant of the parent degradation }\end{array}$ \\
\hline$\beta$ & $\begin{array}{l}\text { the standardization factor, which used to adjust the experimental/modeled light } \\
\text { intensity reported in the referenced paper to our estimated mid-summer (Jun } \\
21 \text { st) daily solar light at } 40^{\circ} \mathrm{N}\end{array}$ \\
\hline
\end{tabular}



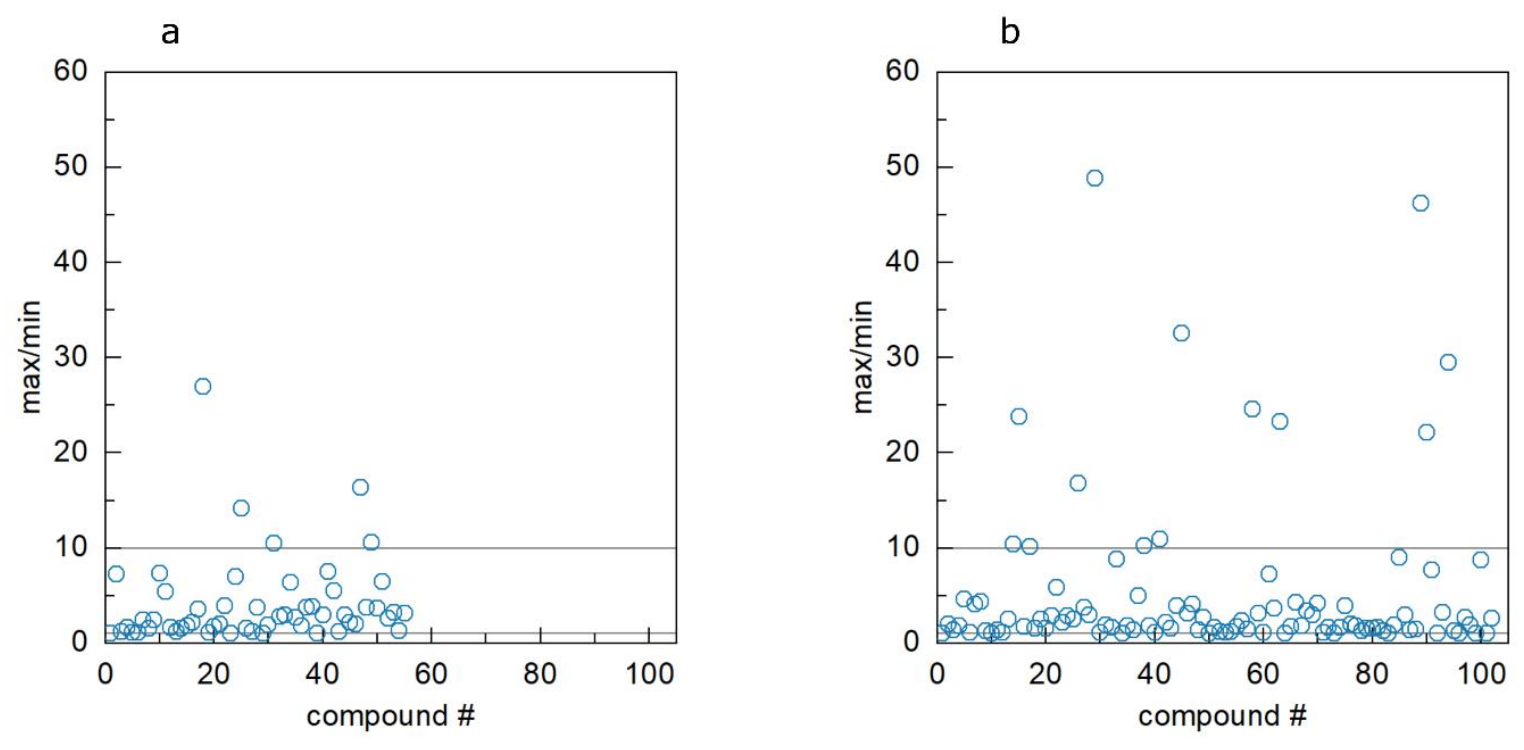

Figure S1. Differences in compiled parent degradation half-lives for the same compound from different studies. a. DB-J-ENV. b. DB-EFSA-ENV. Each compound is represented by one circle in the figure. The difference is calculated as the maximum half-life divided by the minimum half-life ( $\max / \mathrm{min})$. 

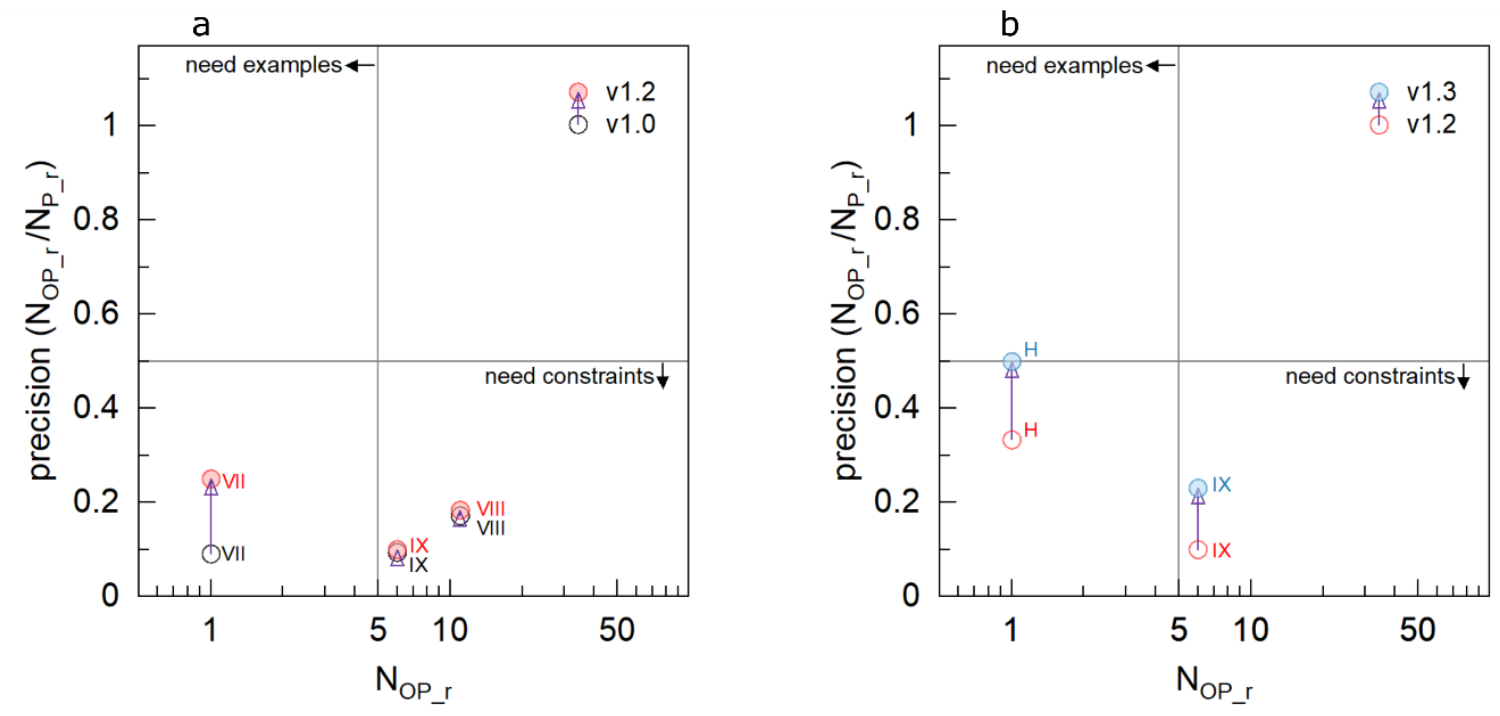

Figure S2. Change in precision for reaction schemes after applying relative reasoning rules (a, from library v1.0 to v1.2) and adding ranking levels (b, from library v1.2 to v1.3) for data from DB-EFSA-ENV. Nop_r stands for number of parent compounds correctly predicted to undergo a certain reaction scheme, $\mathrm{N}_{\mathrm{P}_{-} \mathrm{r}}$ stands for number of parent compounds predicted to undergo the scheme, and precision equals $\mathrm{N}_{\mathrm{OP}_{-} \mathrm{r}} / \mathrm{N}_{\mathrm{P}_{-} \mathrm{r}}$. Reaction schemes with zero $\mathrm{N}_{\mathrm{OP} \_\mathrm{r}}$ or $\mathrm{N}_{\mathrm{P}_{-} \mathrm{r}}$ were not plotted in the figure. Schemes affected by relative reasoning rules were labeled according to the scheme b in Table 2 in the main article: (VII) "aromatic nitro photohydrolysis"; (VIII) "aromatic halide photohydrolysis"; (IX) "aromatic photohydrodehalogenation". Additional schemes affected by ranking are labeled as follows: $(\mathrm{H})$ "phenylurea photochemical N-dealkylation". Note that the labels are consistent with Figure 2 in the main article. 


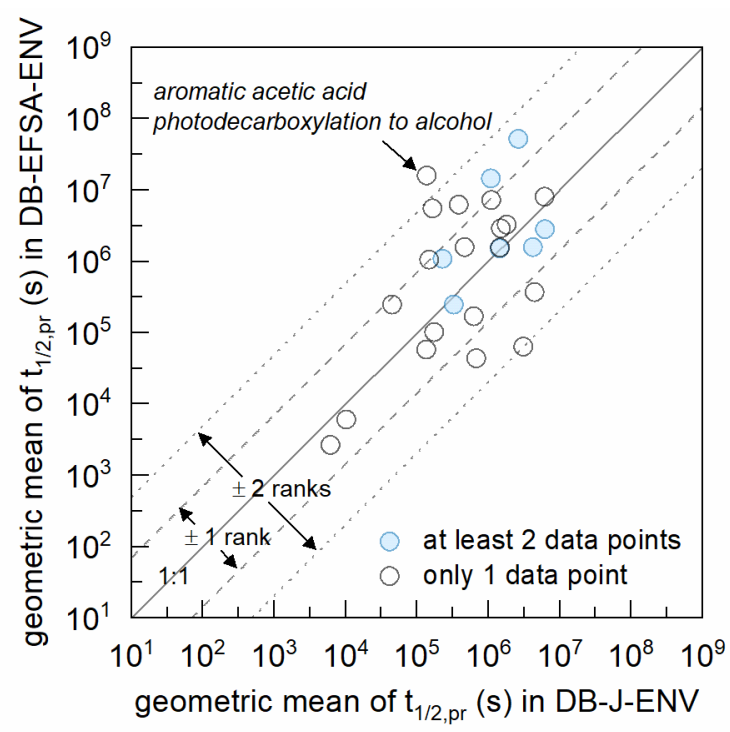

Figure S3. Comparison of the geometric mean of half-lives of the same reaction scheme $\left(t_{1 / 2, \mathrm{pr}}\right)$ in the two databases. 


\section{References}

1. Apell, Jennifer N.; McNeill, K., Updated and validated solar irradiance reference spectra for estimating environmental photodegradation rates. Environmental Science: Processes \& Impacts 2019, 21, (3), 427-437.

2. Yuan, C.; Sleighter, R. L.; Weavers, L. K.; Hatcher, P. G.; Chin, Y.-P., Fast photomineralization of dissolved organic matter in acid mine drainage impacted waters. Environ. Sci. Technol. 2019, 53, (11), 6273-6281.

3. Tebes-Stevens, C.; Patel, J. M.; Jones, W. J.; Weber, E. J., Prediction of hydrolysis products of organic chemicals under environmental ph conditions. Environ. Sci. Technol. 2017, 51, (9), 5008-5016.

4. Pirok, G.; Máté, N.; Varga, J.; Szegezdi, J.; Vargyas, M.; Dóránt, S.; Csizmadia, F., Making "real" molecules in virtual space. J. Chem. Inf. Model. 2006, 46, (2), 563-568.

5. Nilles, G. P.; Zabik, M. J., Photochemistry of bioactive compounds. Multiphase photodegradation of basalin. J. Agric. Food Chem. 1974, 22, (4), 684-688.

6. Wammer, K. H.; Korte, A. R.; Lundeen, R. A.; Sundberg, J. E.; McNeill, K.; Arnold, W. A., Direct photochemistry of three fluoroquinolone antibacterials: Norfloxacin, ofloxacin, and enrofloxacin. Water Res. 2013, 47, (1), 439-448.

7. Fasani, E.; Barberis Negra, F. F.; Mella, M.; Monti, S.; Albini, A., Photoinduced c-f bond cleavage in some fluorinated 7-amino-4-quinolone-3-carboxylic acids. The Journal of Organic Chemistry 1999, 64, (15), 5388-5395.

8. Kusari, S.; Prabhakaran, D.; Lamshöft, M.; Spiteller, M., In vitro residual anti-bacterial activity of difloxacin, sarafloxacin and their photoproducts after photolysis in water. Environ. Pollut. 2009, 157, (10), 2722-2730.

9. Burhenne, J.; Ludwig, M.; Nikoloudis, P.; Spiteller, M., Primary photoproducts and half-lives. Environmental Science and Pollution Research 1997, 4, (1), 10-15.

10. Boreen, A. L.; Arnold, W. A.; McNeill, K., Triplet-sensitized photodegradation of sulfa drugs containing six-membered heterocyclic groups: Identification of an $\mathrm{so} 2$ extrusion photoproduct. Environ. Sci. Technol. 2005, 39, (10), 3630-3638.

11. Boreen, A. L.; Arnold, W. A.; McNeill, K., Photochemical fate of sulfa drugs in the aquatic environment: Sulfa drugs containing five-membered heterocyclic groups. Environ. Sci. Technol. 2004, 38, (14), 3933-3940.

12. Bonvin, F.; Omlin, J.; Rutler, R.; Schweizer, W. B.; Alaimo, P. J.; Strathmann, T. J.; McNeill, K.; Kohn, T., Direct photolysis of human metabolites of the antibiotic sulfamethoxazole: Evidence for abiotic back-transformation. Environ. Sci. Technol. 2013, 47, (13), 6746-6755.

13. Krieger, M. S.; Yoder, R. N.; Gibson, R., Photolytic degradation of florasulam on soil and in water. J. Agric. Food Chem. 2000, 48, (8), 3710-3717.

14. Wilson, R. I.; Mabury, S. A., Photodegradation of metolachlor: Isolation, identification, and quantification of monochloroacetic acid. J. Agric. Food Chem. 2000, 48, (3), 944-950.

15. McConville, M. B.; Hubert, T. D.; Remucal, C. K., Direct photolysis rates and transformation pathways of the lampricides tfm and niclosamide in simulated sunlight. Environ. Sci. Technol. 2016, 50, (18), 9998-10006.

16. Lam, M. W.; Young, C. J.; Mabury, S. A., Aqueous photochemical reaction kinetics and transformations of fluoxetine. Environ. Sci. Technol. 2005, 39, (2), 513-522.

17. Tisler, S.; Zindler, F.; Freeling, F.; Nödler, K.; Toelgyesi, L.; Braunbeck, T.; Zwiener, C., Transformation products of fluoxetine formed by photodegradation in water and biodegradation in zebrafish embryos (danio rerio). Environ. Sci. Technol. 2019, 53, (13), 7400-7409.

18. Carey, J. H.; Fox, M. E., Photodegradation of the lampricide 3-trifluoromethyl-4-nitrophenol (tfm) 1. Pathway of the direct photolysis in solution. J. Great Lakes Res. 1981, 7, (3), 234-241. 
19. Yamada, K.; Terasaki, M.; Makino, M., A novel estrogenic compound transformed from fenthion under uv-a irradiation. J. Hazard. Mater. 2010, 176, (1), 685-691.

20. Weber, J.; Kurková, R.; Klánová, J.; Klán, P.; Halsall, C. J., Photolytic degradation of methylparathion and fenitrothion in ice and water: Implications for cold environments. Environ. Pollut. 2009, 157, (12), 3308-3313.

21. Hirahara, Y.; Ueno, H.; Nakamuro, K., Aqueous photodegradation of fenthion by ultraviolet b irradiation: Contribution of singlet oxygen in photodegradation and photochemical hydrolysis. Water Res. 2003, 37, (2), 468-476.

22. Hirahara, Y.; Ueno, H.; Nakamuro, K., Comparative photodegradation study of fenthion and disulfoton under irradiation of different light sources in liquid- and solid-phases. J. Health Sci. 2001, 47, (2), 129-135.

23. Katagi, T., Photochemistry of organophosphorus herbicide butamifos. J. Agric. Food Chem. 1993, 41, (3), 496-501.

24. Mikami, N.; Imanishi, K.; Yamada, H.; Miyamoto, J., Photodegradation of fenitrothion in water and on soil surface, and its hydrolysis in water. J. Pestic. Sci. 1985, 10, (2), 263-272.

25. Mansour, M.; Thaller, S.; Korte, F., Action of sunlight on parathion. Bull. Environ. Contam. Toxicol. 1983, 30, (1), 358-364.

26. Ivie, G. W.; Bull, D. L., Photodegradation of o-ethyl o-[4-(methylthio)phenyl] s-propyl phosphorodithioate (bay ntn 9306). J. Agric. Food Chem. 1976, 24, (5), 1053-1057.

27. Boulkamh, A.; Harakat, D.; Sehili, T.; Boule, P., Phototransformation of metoxuron [3-(3-chloro4-methoxyphenyl)-1,1-dimethylurea] in aqueous solution. Pest Manage. Sci. 2001, 57, (12), 1119-1126.

28. Tanaka, F. S.; Hoffer, B. L.; Wien, R. G., Photolysis of 3 - (3,4 - dichlorophenyl) - 1, 1 dimethylurea (diuron) in dilute aqueous solution. Toxicol. Environ. Chem. 1986, 11, (4), 261-269.

29. Rosen, J. D.; Strusz, R. F.; Still, C. C., Photolysis of phenylurea herbicides. J. Agric. Food Chem. 1969, 17, (2), 206-207.

30. Pirisi, F. M.; Cabras, P.; Garau, V. L.; Melis, M.; Secchi, E., Photodegradation of pesticides.

Photolysis rates and half-life of pirimicarb and its metabolites in reactions in water and in solid phase. $J$. Agric. Food Chem. 1996, 44, (8), 2417-2422.

31. Temussi, F.; Passananti, M.; Previtera, L.; lesce, M. R.; Brigante, M.; Mailhot, G.; DellaGreca, M., Phototransformation of the drug rivastigmine: Photoinduced cleavage of benzyl-nitrogen sigma bond. J. Photochem. Photobiol. A: Chem. 2012, 239, 1-6.

32. Ruzo, L. O.; Casida, J. E., Photochemistry of thiocarbamate herbicides: Oxidative and free radical processes of thiobencarb and diallate. J. Agric. Food Chem. 1985, 33, (2), 272-276.

33. Draper, W. M.; Crosby, D. G., Photochemistry and volatility of drepamon in water. J. Agric. Food Chem. 1984, 32, (4), 728-733.

34. Vialaton, D.; Richard, C., Direct photolyses of thiobencarb and ethiofencarb in aqueous phase. J. Photochem. Photobiol. A: Chem. 2000, 136, (3), 169-174.

35. Miille, M. J.; Crosby, D. G., Pentachlorophenol and 3,4-dichloroaniline as models for photochemical reactions in seawater. Mar. Chem. 1983, 14, (2), 111-120.

36. Machado, F.; Collin, L.; Boule, P., Photolysis of bromoxynil (3,5-dibromo-4-hydroxybenzonitrile) in aqueous solution. Pestic. Sci. 1995, 45, (2), 107-110.

37. Miller, G. C.; Mulle, M. J.; Crosby, D. G.; Sontum, S.; Zepp, R. G., Photosolvolysis of 3,4dichloroaniline in water: Evidence for an aryl cation intermediate. Tetrahedron 1979, 35, (15), 17971800.

38. Carena, L.; Proto, M.; Minella, M.; Ghigo, G.; Giovannoli, C.; Brigante, M.; Mailhot, G.; Maurino, V.; Minero, C.; Vione, D., Evidence of an important role of photochemistry in the attenuation of the secondary contaminant 3,4-dichloroaniline in paddy water. Environ. Sci. Technol. 2018, 52, (11), 63346342. 
39. Crosby, D. G.; Wong, A. S., Photodecomposition of 2,4,5-trichlorophenoxyacetic acid (2,4,5-t) in water. J. Agric. Food Chem. 1973, 21, (6), 1052-1054.

40. Meunier, L.; Boule, P., Direct and induced phototransformation of mecoprop [2 - (4 - chloro 2 - methylphenoxy)propionic acid] in aqueous solution. Pest Manage. Sci. 2000, 56, (12), 1077-1085.

41. Torrents, A.; Anderson, B. G.; Bilboulian, S.; Johnson, W. E.; Hapeman, C. J., Atrazine photolysis: Mechanistic investigations of direct and nitrate-mediated hydroxy radical processes and the influence of dissolved organic carbon from the chesapeake bay. Environ. Sci. Technol. 1997, 31, (5), 1476-1482.

42. Ruggeri, G.; Ghigo, G.; Maurino, V.; Minero, C.; Vione, D., Photochemical transformation of ibuprofen into harmful 4-isobutylacetophenone: Pathways, kinetics, and significance for surface waters. Water Res. 2013, 47, (16), 6109-6121.

43. Poiger, T.; Buser, H.-R.; Müller, M. D., Photodegradation of the pharmaceutical drug diclofenac in a lake: Pathway, field measurements, and mathematical modeling. Environ. Toxicol. Chem. 2001, 20, (2), 256-263.

44. Eriksson, J.; Svanfelt, J.; Kronberg, L., A photochemical study of diclofenac and its major transformation products. Photochem. Photobiol. 2010, 86, (3), 528-532.

45. Kotnik, K.; Kosjek, T.; Žegura, B.; Filipič, M.; Heath, E., Photolytic fate and genotoxicity of benzophenone-derived compounds and their photodegradation mixtures in the aqueous environment. Chemosphere 2016, 147, 114-123.

46. Packer, J. L.; Werner, J. J.; Latch, D. E.; McNeill, K.; Arnold, W. A., Photochemical fate of pharmaceuticals in the environment: Naproxen, diclofenac, clofibric acid, and ibuprofen. Aquat. Sci. 2003, 65, (4), 342-351.

47. DellaGreca, M.; Brigante, M.; Isidori, M.; Nardelli, A.; Previtera, L.; Rubino, M.; Temussi, F., Phototransformation and ecotoxicity of the drug naproxen-na. Environ. Chem. Lett. 2004, 1, (4), 237241.

48. Temussi, F.; Cermola, F.; DellaGreca, M.; lesce, M. R.; Passananti, M.; Previtera, L.; Zarrelli, A., Determination of photostability and photodegradation products of indomethacin in aqueous media. $J$. Pharm. Biomed. Anal. 2011, 56, (4), 678-683.

49. Wammer, K. H.; Anderson, K. C.; Erickson, P. R.; Kliegman, S.; Moffatt, M. E.; Berg, S. M.; Heitzman, J. A.; Pflug, N. C.; McNeill, K.; Martinovic-Weigelt, D.; Abagyan, R.; Cwiertny, D. M.; Kolodziej, E. P., Environmental photochemistry of altrenogest: Photoisomerization to a bioactive product with increased environmental persistence via reversible photohydration. Environ. Sci. Technol. 2016, 50, (14), 7480-7488.

50. Kolodziej, E. P.; Qu, S.; Forsgren, K. L.; Long, S. A.; Gloer, J. B.; Jones, G. D.; Schlenk, D.; Baltrusaitis, J.; Cwiertny, D. M., Identification and environmental implications of photo-transformation products of trenbolone acetate metabolites. Environ. Sci. Technol. 2013, 47, (10), 5031-5041.

51. Pflug, N. C.; Hankard, M. K.; Berg, Stephanie M.; O'Connor, M.; Gloer, J. B.; Kolodziej, E. P.; Cwiertny, D. M.; Wammer, K. H., Environmental photochemistry of dienogest: Phototransformation to estrogenic products and increased environmental persistence via reversible photohydration.

Environmental Science: Processes \& Impacts 2017, 19, (11), 1414-1426.

52. Kliegman, S.; Eustis, S. N.; Arnold, W. A.; McNeill, K., Experimental and theoretical insights into the involvement of radicals in triclosan phototransformation. Environ. Sci. Technol. 2013, 47, (13), 67566763.

53. Buth, J. M.; Grandbois, M.; Vikesland, P. J.; McNeill, K.; Arnold, W. A., Aquatic photochemistry of chlorinated triclosan derivatives: Potential source of polychlorodibenzo-p-dioxins. Environ. Toxicol.

Chem. 2009, 28, (12), 2555-2563.

54. Latch, D. E.; Packer, J. L.; Arnold, W. A.; McNeill, K., Photochemical conversion of triclosan to 2,8dichlorodibenzo-p-dioxin in aqueous solution. J. Photochem. Photobiol. A: Chem. 2003, 158, (1), 63-66. 
55. Steen, P. O.; Grandbois, M.; McNeill, K.; Arnold, W. A., Photochemical formation of halogenated dioxins from hydroxylated polybrominated diphenyl ethers (oh-pbdes) and chlorinated derivatives (ohpbcdes). Environ. Sci. Technol. 2009, 43, (12), 4405-4411.

56. Zhang, Y.-n.; Xie, Q.; Sun, G.; Yang, K.; Song, S.; Chen, J.; Zhou, C.; Li, Y., Effects of dissolved organic matter on phototransformation rates and dioxin products of triclosan and 2' -ho-bde-28 in estuarine water. Environmental Science: Processes \& Impacts 2016, 18, (9), 1177-1184.

57. Kim, M.; O'Keefe, P. W., Photodegradation of polychlorinated dibenzo-p-dioxins and dibenzofurans in aqueous solutions and in organic solvents. Chemosphere 2000, 41, (6), 793-800.

58. Dung, M. H.; O'Keefe, P. W., Comparative rates of photolysis of polychlorinated dibenzofurans in organic solvents and in aqueous solutions. Environ. Sci. Technol. 1994, 28, (4), 549-554.

59. Vialaton, D.; Richard, C., Phototransformation of aromatic pollutants in solar light: Photolysis versus photosensitized reactions under natural water conditions. Aquat. Sci. 2002, 64, (2), 207-215.

60. Vialaton, D.; Baglio, D.; Paya-Perez, A.; Richard, C., Photochemical transformation of acifluorfen under laboratory and natural conditions. Pest Manage. Sci. 2001, 57, (4), 372-379.

61. Aguer, J. P.; Boule, P.; Bonnemoy, F.; Chezal, J. M., Phototransformation of napropamide [n, $n$ diethyl-2-(1-naphthyloxy)propionamide] in aqueous solution: Influence on the toxicity of solutions. Pestic. Sci. 1998, 54, (3), 253-257.

62. Chang, L. L.; Giang, B. Y.; Lee, K. S.; Tseng, C. K., Aqueous photolysis of napropamide. J. Agric. Food Chem. 1991, 39, (3), 617-621.

63. Oyamada, M.; Kuwatsuka, S., Photodegradation of the herbicide naproanilide in aqueous solution and in surface water of flooded soil. J. Pestic. Sci. 1986, 11, (2), 179-187.

64. Adachi, T.; Suzuki, Y.; Nishiyama, M.; Kodaka, R.; Fujisawa, T.; Katagi, T., Photodegradation of strobilurin fungicide mandestrobin in water. J. Agric. Food Chem. 2018, 66, (32), 8514-8521.

65. Wei-Haas, M. L. The influence of dissolved organic matter on the fate of polybrominated diphenyl ethers (pbdes) in the environment. The Ohio State University, 2015.

66. Halasz, A.; Hawari, J.; Perreault, N. N., New insights into the photochemical degradation of the insensitive munition formulation imx-101 in water. Environ. Sci. Technol. 2018, 52, (2), 589-596.

67. Haag, W. R.; Spanggord, R.; Mill, T.; Podoll, R. T.; Chou, T.-W.; Tse, D. S.; Harper, J. C., Aquatic environmental fate of nitroguanidine. Environ. Toxicol. Chem. 1990, 9, (11), 1359-1367.

68. Rao, B.; Wang, W.; Cai, Q.; Anderson, T.; Gu, B., Photochemical transformation of the insensitive munitions compound 2,4-dinitroanisole. Sci. Total Environ. 2013, 443, (Supplement C), 692-699.

69. Vione, D.; De Laurentiis, E.; Berto, S.; Minero, C.; Hatipoglu, A.; Cinar, Z., Modeling the photochemical transformation of nitrobenzene under conditions relevant to sunlit surface waters: Reaction pathways and formation of intermediates. Chemosphere 2016, 145, 277-283.

70. McFall, A. S.; Anastasio, C., Photon flux dependence on solute environment in water ices. Environmental Chemistry 2016, 13, (4), 682-687.

71. Plumlee, M. H.; Reinhard, M., Photochemical attenuation of n-nitrosodimethylamine (ndma) and other nitrosamines in surface water. Environ. Sci. Technol. 2007, 41, (17), 6170-6176.

72. Williams, K. L.; Kaur, R.; McFall, A. S.; Kalbfleisch, J.; Gladfelder, J. J.; Ball, D. B.; Anastasio, C.; Tjeerdema, R. S., Aqueous photolysis of benzobicyclon hydrolysate. J. Agric. Food Chem. 2018, 66, (22), 5462-5472.

73. Nishiyama, M.; Suzuki, Y.; Katagi, T., Hydrolysis and photolysis of insecticide metofluthrin in water. J. Pestic. Sci. 2010, 35, (4), 447-455.

74. Katagi, T., Photodegradation of 3-phenoxybenzoic acid in water and on solid surfaces. J. Agric. Food Chem. 1992, 40, (7), 1269-1274.

75. Wamhoff, H.; Schneider, V., Photodegradation of imidacloprid. J. Agric. Food Chem. 1999, 47, (4), 1730-1734. 
76. Mulligan, R. A.; Redman, Z. C.; Keener, M. R.; Ball, D. B.; Tjeerdema, R. S., Photodegradation of clothianidin under simulated california rice field conditions. Pest Manage. Sci. 2015, 72, (7), 1322-1327.

77. De Laurentiis, E.; Chiron, S.; Kouras-Hadef, S.; Richard, C.; Minella, M.; Maurino, V.; Minero, C.; Vione, D., Photochemical fate of carbamazepine in surface freshwaters: Laboratory measures and modeling. Environ. Sci. Technol. 2012, 46, (15), 8164-8173.

78. Lam, M. W.; Mabury, S. A., Photodegradation of the pharmaceuticals atorvastatin, carbamazepine, levofloxacin, and sulfamethoxazole in natural waters. Aquat. Sci. 2005, 67, (2), 177-188.

79. West, C. E.; Rowland, S. J., Aqueous phototransformation of diazepam and related human metabolites under simulated sunlight. Environ. Sci. Technol. 2012, 46, (9), 4749-4756.

80. Kramer, J. B.; Canonica, S.; Hoigné, J.; Kaschig, J., Degradation of fluorescent whitening agents in sunlit natural waters. Environ. Sci. Technol. 1996, 30, (7), 2227-2234.

81. DellaGreca, M.; lesce, M. R.; Previtera, L.; Rubino, M.; Barone, V.; Crescenzi, O., Phototransformation of the drug trazodone in aqueous solution. J. Photochem. Photobiol. A: Chem. 2008, 199, (2), 353-357.

82. Astarita, A.; DellaGreca, M.; lesce, M. R.; Montanaro, S.; Previtera, L.; Temussi, F., Polycyclic compounds by sunlight exposure of the drug rosuvastatin in water. J. Photochem. Photobiol. A: Chem. 2007, 187, (2), 263-268.

83. Cermola, F.; DellaGreca, M.; lesce, M. R.; Montanaro, S.; Previtera, L.; Temussi, F.; Brigante, M., Irradiation of fluvastatin in water: Structure elucidation of photoproducts. J. Photochem. Photobiol. A: Chem. 2007, 189, (2), 264-271.

84. Zepp, R. G.; Gumz, M. M.; Miller, W. L.; Gao, H., Photoreaction of valerophenone in aqueous solution. The Journal of Physical Chemistry $A$ 1998, 102, (28), 5716-5723.

85. Ware, G. W.; Crosby, D. G.; Giles, J. W., Photodecomposition of dda. Arch. Environ. Contam. Toxicol. 1980, 9, (2), 135-146.

86. Huang, J.; Mabury, S. A., The role of carbonate radical in limiting the persistence of sulfurcontaining chemicals in sunlit natural waters. Chemosphere 2000, 41, (11), 1775-1782.

87. Brigante, M.; DellaGreca, M.; Previtera, L.; Rubino, M.; Temussi, F., Degradation of hydrochlorothiazide in water. Environ. Chem. Lett. 2005, 2, (4), 195-198.

88. Sharma, A. K.; Zimmerman, W. T.; Singles, S. K.; Malekani, K.; Swain, S.; Ryan, D.; McQuorcodale, G.; Wardrope, L., Photolysis of chlorantraniliprole and cyantraniliprole in water and soil: Verification of degradation pathways via kinetics modeling. J. Agric. Food Chem. 2014, 62, (28), 6577-6584.

89. DellaGreca, M.; lesce, M. R.; Isidori, M.; Montanaro, S.; Previtera, L.; Rubino, M., Phototransformation of amlodipine in aqueous solution: Toxicity of the drug and its photoproduct on aquatic organisms. International Journal of Photoenergy 2007, 2007.

90. DellaGreca, M.; lesce, M. R.; Cermola, F.; Rubino, M.; Isidori, M., Phototransformation of carboxin in water. Toxicity of the pesticide and its sulfoxide to aquatic organisms. J. Agric. Food Chem. 2004, 52, (20), 6228-6232.

91. Yuan, C.; Tebes-Stevens, C.; Weber, E. J., Reaction library to predict direct photochemical transformation products of environmental organic contaminants in sunlit aquatic systems. Environ. Sci. Technol. 2020, 54, (12), 7271-7279. 